\title{
Morphology of Ion-Sputtered Surfaces
}

\author{
Maxim Makeev ${ }^{\dagger}$, Rodolfo Cuerno ${ }^{\ddagger}$, and Albert-László Barabási ${ }^{\dagger}$ \\ $\dagger$ Department of Physics, University of Notre Dame, Notre Dame, IN 46556, USA \\ ¥Departamento de Matemáticas and Grupo Interdisciplinar de Sistemas Complicados, Universidad Carlos III de Madrid, \\ Avenida de la Universidad 30, 28911 Leganés, SPAIN
}

(February 1, 2008)

\begin{abstract}
We derive a stochastic nonlinear continuum theory to describe the morphological evolution of amorphous surfaces eroded by ion bombardment. Starting from Sigmund's theory of sputter erosion, we calculate the coefficients appearing in the continuum equation in terms of the physical parameters characterizing the sputtering process. We analyze the morphological features predicted by the continuum theory, comparing them with the experimentally reported morphologies. We show that for short time scales, where the effect of nonlinear terms is negligible, the continuum theory predicts ripple formation. We demonstrate that in addition to relaxation by thermal surface diffusion, the sputtering process can also contribute to the smoothing mechanisms shaping the surface morphology. We explicitly calculate an effective surface diffusion constant characterizing this smoothing effect, and show that it is responsible for the low temperature ripple formation observed in various experiments. At long time scales the nonlinear terms dominate the evolution of the surface morphology. The nonlinear terms lead to the stabilization of the ripple wavelength and we show that, depending on the experimental parameters such as angle of incidence and ion energy, different morphologies can be observed: asymptotically, sputter eroded surfaces could undergo kinetic roughening, or can display novel ordered structures with rotated ripples. Finally, we discuss in detail the existing experimental support for the proposed theory, and uncover novel features of the surface morphology and evolution, that could be directly tested experimentally.
\end{abstract}

PACS numbers: 79.20.Rf, 64.60.Ht, 68.35.Rh

\section{INTRODUCTION}

Sputtering is the removal of material from the surface of solids through the impact of energetic particles [1] 3 . It is a widespread experimental technique, used in a large number of applications with a remarkable level of sophistication. It is a basic tool in surface analysis, depth profiling, sputter cleaning, micromachining, and sputter deposition. Perhaps the largest community of users is in the thin film and semiconductor fabrication areas, sputter erosion being routinely used for etching patterns important to the production of integrated circuits and device packaging.

To have a better control over this important tool, we need to understand the effect of the sputtering process on the surface morphology. In many cases sputtering is routinely used to smooth out surface features. On the other hand, some investigations indicate that sputtering can also roughen the surface. Consequently, sputter erosion may have different effects on the surface, depending on many factors, such as incident ion energy, mass, angle of incidence, sputtered substrate temperature and material composition. The experimental results on the effect of sputter erosion on the surface morphology can be classified in two main classes. There exists ample experimental evidence that ion sputtering can lead to the development of periodic ripples on the surface 四 27]. Depending on the sputtered substrate and the sputtering conditions these ripples can be surprisingly straight and ordered. However, a number of recent investigations 28 36] have provided rather detailed and convincing experimental evidence that under certain experimental conditions ion eroded surfaces become rough, and the roughness follows the predictions of various scaling theories 37. Moreover, these investigations did not find any evidence of ripple formation on the surface. Up to recently these two morphological features were treated separately and no unified theoretical framework describing these morphologies was available.

The first widely accepted theoretical approach describing the process of ripple formation on amorphous substrates was developed by Bradley and Harper $(\mathrm{BH})$ [38. This theory is rather successful in predicting the ripple wavelength and orientation in agreement with numerous experimental observations. However, a number of experimental results have systematically eluded this theory. For example, the $\mathrm{BH}$ theory predicts an unlimited exponential increase in ripple amplitude in contrast with the observed saturation of the surface width. Similarly, it cannot account for surface roughening, or for ripple orientations different from those defined by the incoming ion direction or perpendicular to it. Finally, recent experiments [12,13 have observed ripples whose wavelength is independent of the substrate temperature, and linear in the ion energy, in contrast with the $\mathrm{BH}$ prediction of a ripple wavelength which depends exponentially with temperature and decreases with ion energy.

In the light of the accumulated experimental results, it is clear that a theory going beyond the $\mathrm{BH}$ approach is required, motivating the results described in this pa- 
per. Thus here we investigate the morphology of ionsputtered amorphous surfaces aiming to describe in an unified framework the dynamic and scaling behavior of the experimentally observed surface morphologies. For this we derive a nonlinear theory that describes the time evolution of the surface morphology. At short time scales the nonlinear theory predicts the development of a periodic ripple structure, while at large time scales the surface morphology may be either rough or dominated by new ripples, that are different from those existing at short time scales. We find that transitions may take place between various surface morphologies as the experimental parameters (e.g. angle of incidence, penetration depth) are varied. Usually stochastic equations describing growth and erosion models are constructed using symmetry arguments and conservation laws. In contrast, here we show that for sputter eroded surfaces the growth equation can be derived directly from a microscopic model of the elementary processes taking place in the system. A particular case of our theory was presented in 39]. In addition, we show that the presented theory can be extended to describe low temperature ripple formation as well. We demonstrate that under certain conditions ion-sputtering can lead to preferential erosion that appears as a surface diffusion term in the equation of motion even though no mass transport along the surface takes place in the system. To distinguish it from ordinary surface diffusion, in the following we refer to this phenomenon as effective smoothing (ES). We calculate analytically an effective surface diffusion constant accounting for the ES effect, and study its dependence on the ion energy, flux, angle of incidence, and penetration depth. The effect of ES on the morphology of ion-sputtered surfaces is summarized in a morphological phase diagram, allowing for direct experimental verification of our predictions. A restricted study along these lines appeared in 40].

The paper is organized as follows. In Section II we review the recent advances in the scaling theory of rough (self-affine) interfaces. Section III is dedicated to a brief overview of the experimental results on surface morphology development under ion sputtering. A short summary of the theoretical approaches developed to describe the morphology of ion sputtered surfaces is presented in Section IV. This section also contains a short description of Sigmund's theory of sputtering, that is the basis for our calculations. In Section $\mathrm{V}$ we derive the nonlinear stochastic equation describing sputter erosion. Analysis of this equation is presented in Section VI, discussing separately both the high and low temperature ripple formation. We compare the predictions of our theory with experimental results on surface roughening and ripple formation in Section VII, followed by Section VIII, that summarizes our findings.

\section{SCALING THEORY}

In the last decade we witnessed the development of an array of theoretical tools and techniques intended to describe and characterize the roughening of nonequilibrium surfaces and interfaces [37. Initiated by advances in understanding the statistical mechanics of various nonequilibrium systems, it has been observed that the roughness of many natural surfaces follows rather simple scaling laws, which can be quantified using scaling exponents. Since kinetic roughening is a common feature of ionbombarded surfaces, before we discuss the experimental results on sputtering, we need to introduce the main quantities characterizing the surface morphology.

Let us consider a two-dimensional surface that is characterized by the height function $h(x, y, t)$. The morphology and dynamics of a rough surface can be quantified by the interface width, defined by the rms fluctuations in the height $h(x, y, t)$,

$$
w(L, t) \equiv \sqrt{\frac{1}{L^{2}} \sum_{x, y=1, L}[h(x, y)-\bar{h}]^{2}},
$$

where $L$ is the linear size of the sample and $\bar{h}$ is the mean surface height of the surface

$$
\bar{h}(t) \equiv \frac{1}{L^{2}} \sum_{x, y=1, L} h(x, y, t) .
$$

Instead of measuring the roughness of a surface over the whole sample size $L \times L$, we can choose a window of size $\ell \times \ell$, and measure the local width, $w(\ell)$. A general property of many rough surfaces is that the roughness depends on the length scale of observation. This can be quantified by plotting $w(\ell)$ as a function of $\ell$. There are two characteristic regimes one can distinguish:

(i) For length scales smaller than $\ell_{\times}$, the local width increases as

$$
w(\ell) \sim \ell^{\alpha},
$$

where $\alpha$ is the roughness exponent. If we are interested in surface phenomena that take place at length scales shorter than $\ell_{\times}$then we cannot neglect the roughness of the surface. In this regime the roughness is not simply a number, but it depends on the length scale accessible to the method probing the surface.

(ii) For $\ell \gg \ell_{x}, w(\ell)$ is independent of $\ell$, thus, at length scales larger than $\ell_{\times}$, the surface is smooth. In this regime we can characterize the surface roughness with the saturation width $w_{\text {sat }}=w\left(\ell_{\times}\right)$.

The dynamics of the roughening process can be best characterized by the time dependent total width (11). At early times the total width increases as $w(L, t) \sim t^{\beta}$, where $\beta$ is the growth exponent. However, for finite systems, after a crossover time $t_{\times}$, the width saturates, following the Family-Vicsek scaling function [41]

$$
w(L, t) \equiv t^{\beta} g\left(\frac{t}{L^{z}}\right),
$$


where $z=\alpha / \beta$ is the dynamic exponent and $g(u \ll 1) \sim$ 1 , while $g(u \gg 1) \sim u^{-\beta}$.

Scaling relations such as Eq. (田) allow us to define universality classes. The universality class concept is a product of modern statistical mechanics, and encodes the fact that there are but a few essential factors that determine the exponents characterizing the scaling behavior. Thus different systems, which at first sight may appear to have no connection between them, behave in a remarkably similar fashion. The values of the exponents $\alpha$ and $\beta$ are independent of many "details" of the system, and they are uniquely defined for a given universality class. In contrast, other quantities, such as $A, \ell_{\times}$, or $w_{\text {sat }}$, are non-universal, i.e. they depend on almost every detail of the system.

\section{EXPERIMENTAL RESULTS}

The morphology of surfaces bombarded by energetic ions has long fascinated the experimental community. Lately, with the development of high resolution observation techniques such as atomic force (AFM) and scanning tunneling (STM) microscopies, this problem is living a new life. The various experimental investigations can be classified into two main classes. First, early investigations, corroborated by numerous recent studies, have found that sputter eroded surfaces develop a ripple morphology with a rather characteristic wavelength of the order of a few micrometers 4 27. However, a number of research groups have found no evidence of ripples, but observed the development of apparently random, rough surfaces [28 36], that were characterized using scaling theories. In the following we summarize the key experimental observations for both ripple development and kinetic roughening.

\section{A. Ripple formation}

The ripple morphology of ion bombarded surfaces has been initially discovered in the mid 1970's 目 6 . Since then, a number of research groups have provided detailed quantitative results regarding the ripple characteristics and dynamics of ripple formation. It is beyond the scope of this paper to offer a comprehensive review of the vast body of the experimental literature on the subject. Thus, we selected a few experiments that offer a representative picture of the experimental features that appear to be common to different materials.

Angle of incidence: An experimental parameter which is rather easy to change in sputtering is the angle of incidence $\theta$ of the incoming ions relative to the normal to the average surface configuration. Consequently, numerous research groups have investigated the effect of $\theta$ on the ripples. These results indicate that ripples appear only for a limited range of incidence angles, which, depending on materials and ions involved, typically vary between $30^{\circ}$ and $60^{\circ}$.

Support for a well defined window in $\theta$ for ripple formation was offered by Stevie et al. [7], who observed abrupt secondary ion yield changes (correlated with the onset of ripple morphology development) in experiments on 6 and $8 \mathrm{keV} \mathrm{O}_{2}^{+}$sputtering of $\mathrm{Si}$ and $8,5.5$, and $2.5 \mathrm{keV}$ $\mathrm{O}_{2}^{+}$sputtering of GaAs at incidence angles between $39^{\circ}$ and $52^{\circ}$. These results were corroborated by Karen et al. [8 [10], who investigated ripple formation on GaAs surfaces under bombardment with $10.5 \mathrm{keV} \mathrm{O}_{2}^{+}$ions. They found that ripple formation takes place for angles of incidence between $30^{\circ}$ and $60^{\circ}$ (see Table I of Ref. [10]). Similarly, Wittmaack 11] found that ripple formation occurs at incidence angles between $32^{\circ}$ and $58^{\circ}$ during 10 $\mathrm{keV} \mathrm{O}_{2}^{+}$-ion bombardment of a $\mathrm{Si}$ target.

Temperature dependence: Another parameter that has been found to influence the ripple structure, and in particular the ripple wavelength, is the temperature of the substrate, $T$. Two different behaviors have been documented: exponential dependence of the ripple wavelength on $T$ has been observed at high temperatures, while the wavelength was found to be constant at low temperatures.

A series of experiments on the temperature dependence of ripple formation were reported by MacLaren et al. 12. They studied InP and GaAs surfaces bombarded with $17.5 \mathrm{KeV} \mathrm{Cs}^{+}$and $5.5 \mathrm{keV} \mathrm{O}_{2}^{+}$ion beams in the temperature range from $-50^{\circ} \mathrm{C}$ to $200^{\circ} \mathrm{C}$. For GaAs bombarded by $\mathrm{Cs}^{+}$ions the ripple wavelength increased from $0.89 \mu \mathrm{m}$ to $2.1 \mu \mathrm{m}$ as the temperature increased from $0^{\circ} \mathrm{C}$ to $100^{\circ} \mathrm{C}$. Probably the most interesting finding of their study was that lowering the temperature, the ripple wavelength did not go continuously to zero as one would expect, since the surface diffusion constant decreases exponentially with temperature (see Sect. IV C), but rather at approximately $60^{\circ} \mathrm{C}$ it stabilized at a constant value. MacLaren et al. interpreted this as the emergence of radiation enhanced diffusion, that gives a constant (temperature independent) contribution to the diffusion constant. Recently, Umbach et al. 13] have studied the sputter-induced ripple formation on $\mathrm{SiO}_{2}$ surfaces using $0.5-2.0 \mathrm{keV} \mathrm{Ar}$ ion beams. The temperature dependence of the ripple wavelength $\ell$ was investigated for temperatures ranging from room temperature to $800^{\circ} \mathrm{C}$. It was found that for high temperatures, $T \geq 400^{\circ} \mathrm{C}$, the ripple wavelength follows the Arrhenius law $\left(1 / T^{1 / 2}\right) \exp \left(-\Delta E / 2 k_{B} T\right)$, indicating the thermally activated character of the relaxation processes. However, at low temperatures the ripple wavelength was independent of temperature, indicating the presence of a temperature independent relaxation mechanism.

Results indicating temperature independent nondiffusive relaxation have been reported for crystalline materials as well by Carter et al. [14]. In these experiments Si bombarded with highly energetic $10-40 \mathrm{keV} \mathrm{Xe}^{+}$-ions led to ripple formation with wavelength $\ell \simeq 0.4 \mu \mathrm{m}$ for 
angles of incidence close to $45^{\circ}$. Changing the surface temperature from $100 \mathrm{~K}$ to $300 \mathrm{~K}$ the ripple wavelength and orientation did not change. This observation led the authors to conclude that the smoothing mechanism is not of thermal origin. They also found that the ripple wavevector is relatively insensitive to the primary ion energy.

Flux and fluence dependence: The effect of the flux on the surface dynamics was studied by Chason et al. [15, 16]. In these experiments a $1 \mathrm{keV}$ Xe ion beam was directed towards a $\mathrm{SiO}_{2}$ sample with an angle of incidence of $55^{\circ}$. The typical incoming flux was $10^{13} \mathrm{~cm}^{-2} \mathrm{~s}^{-1}$ and fluence (the number of incoming atoms per surface area, playing the role of time) was up to $10 \times 10^{15} \mathrm{~cm}^{-2}$. The surface was analyzed using in situ energy dispersive X-ray reflectivity and ex situ AFM. It was found that the interface roughness, which is proportional to the ripple amplitude, increases linearly with the fluence. Similar experiments were performed on $\mathrm{Ge}(001)$ surfaces 17 using $0.3,0.5$, and $1 \mathrm{keV}$ Xe ion beams for $T=350^{\circ} \mathrm{C}$. For flux values up to $3 \mu \mathrm{A} / \mathrm{cm}^{2}$ and fluences up to $6 \times 10^{16} \mathrm{~cm}^{-2}$, the roughness is seen to increase as the square of the flux.

Ion energy: The ripple wavelength dependence on the incident ion energy and the angle of incidence was reported in Refs. [8 10]. The experiments indicate that the ripple wavelength is linear in the energy, following $\ell \sim \epsilon \cos \theta$. Similar results were obtained in Ref. [18], providing an extensive study of ripple formation by secondary ion spectrometry and scanning electron microscopy. The ripple topography was observed during $\mathrm{O}_{2}^{+}$ primary ion bombardment of a $\mathrm{Si}(100)$ substrate with ion energies ranging between $1.5 \mathrm{keV}$ and $9 \mathrm{keV}$. No ripples were observed for energies less than $1.5 \mathrm{keV}$ or for high energies, such as $1.5 \mathrm{keV}$ and $7 \mathrm{keV}$, when $\mathrm{Ar}^{+}$primary ions were used. The experiments indicate that the ripple wavelength increases linearly from 100 to $400 \mathrm{~nm}$ when the energy of the primary ion changes from 1 to $9 \mathrm{keV}$. Furthermore, the experimental data indicated that the primary ion penetration depth $a$ and the ripple wavelength $\ell$ are related by the empirical relation $\ell=40 a$. The wavelength of the ripples is found to be independent of the primary ion flux and dependent merely on fluence, i.e. sputtered depth. The recent results by Umbach et al. [13] provided further strong evidence for the linear relationship between the ion energy and the ripple wavelength for $\mathrm{SiO}_{2}$ substrates (see below).

Ripple amplitude: Indirect results on the ripple amplitude were presented by Vajo et al. [18] in their study of the surface topography induced secondary ion yield changes on $\mathrm{SiO}_{2}$ surfaces bombarded by $\mathrm{O}_{2}^{+}$ions. The authors have found that the yield changes exponentially in the first stages of ripple development and saturates for large sputtered depth. Direct evidence on ripple amplitude saturation was obtained by Erlebacher et al. [20], who measured the time evolution of the ripple amplitude in experiments bombarding $\mathrm{Si}(100)$ surfaces with 0.75 $\mathrm{keV} \mathrm{Ar}{ }^{+}$ions. They found that, while at short times the ripple amplitude increases exponentially, it saturates

\begin{tabular}{|c|c|c|c|c|c|}
\hline Material & $\begin{array}{l}\text { Ion } \\
\text { type }\end{array}$ & $\begin{array}{c}\text { Angle } \\
\text { of } \\
\text { incidence }\end{array}$ & $\begin{array}{c}\text { Ion energy } \\
(\mathrm{keV})\end{array}$ & $\begin{array}{c}\text { Ripple } \\
\text { wavelength } \\
(\mu \mathrm{m})\end{array}$ & Ref. \\
\hline $\operatorname{GaAs}(100)$ & $\mathrm{O}_{2}^{+}$ & $39^{\circ}$ & 8 & \begin{tabular}{ll|}
0.2 \\
\end{tabular} & (7) \\
\hline$\overline{\operatorname{GaAs}(100)}$ & $\mathrm{O}_{2}^{+}$ & $42^{\circ}$ & 5.5 & 0.1 & (7) \\
\hline$\overline{\operatorname{GaAs}(100)}$ & $\mathrm{O}_{2}^{+}$ & $37^{\circ}$ & 10.5 & 0.23 & 9 \\
\hline $\operatorname{GaAs}(100)$ & $\mathrm{O}_{2}^{+}$ & $42^{\circ}$ & 5.5 & 0.13 & 10 \\
\hline$\overline{\operatorname{GaAs}(100)}$ & $\mathrm{O}_{2}^{+}$ & $39^{\circ}$ & 8.0 & 0.21 & 10 \\
\hline $\operatorname{GaAs}(100)$ & $\mathrm{O}_{2}^{+}$ & $37^{\circ}$ & 10.5 & 0.27 & 10 \\
\hline$\overline{\operatorname{GaAs}(100)}$ & $\mathrm{O}_{2}^{+}$ & $57^{\circ}$ & 13 & 0.33 & 10 \\
\hline GaAs & $\mathrm{O}_{2}^{+}$ & $40^{\circ}$ & 3.0 & 0.075 & 26 \\
\hline GaAs & $\mathrm{O}_{2}^{+}$ & $40^{\circ}$ & 7.0 & 0.130 & 26 \\
\hline $\mathrm{Ge}(001)$ & $\mathrm{Xe}^{+}$ & $55^{\circ}$ & 1 & 0.2 & 15 \\
\hline $\mathrm{Si}(001)$ & $\mathrm{O}_{2}^{+}$ & $41^{\circ}$ & 6 & 0.4 & (7) \\
\hline $\mathrm{Si}(001)$ & $\mathrm{O}_{2}^{+}$ & $42^{\circ}$ & 5.5 & 0.5 & [7] \\
\hline $\mathrm{Si}(100)$ & $\mathrm{O}_{2}^{+}$ & $39^{\circ}$ & 8 & 0.5 & (7) \\
\hline$\overline{\mathrm{Si}(100)}$ & $\mathrm{O}_{2}^{+}$ & $40^{\circ}$ & 3 & 0.198 & 18] \\
\hline$\overline{\mathrm{Si}(100)}$ & $\mathrm{O}_{2}^{+}$ & $40^{\circ}$ & 5 & 0.302 & 18 \\
\hline $\begin{array}{l}\mathrm{Si}(100) \\
\end{array}$ & $\mathrm{O}_{2}^{+}$ & $40^{\circ}$ & 9 & 0.408 & 18 \\
\hline$\overline{\mathrm{Si}(100)}$ & $\mathrm{Ar}^{+}$ & $67.5^{\circ}$ & 0.75 & 0.57 & 19 \\
\hline $\mathrm{Si}$ & $\mathrm{Xe}^{+}$ & $45^{\circ}$ & 40 & 0.4 & 14 \\
\hline $\mathrm{Si}$ & $\mathrm{O}_{2}^{+}$ & $37^{\circ}$ & 12.5 & 0.35 & 21] \\
\hline $\mathrm{SiO}_{2}$ & $\mathrm{Ar}^{+}$ & $45^{\circ}$ & $0.5-2$ & $0.2-0.55$ & 13 \\
\hline $\mathrm{SiO}_{2}$ & $\mathrm{Xe}^{+}$ & $55^{\circ}$ & 1 & 0.03 & 16 \\
\hline
\end{tabular}

TABLE I. Summary of the ripple characteristics reported in sputter erosion experiments of non-metallic substrates. In all cases shown, the ripple wave vector is parallel to the ion beam direction. Note that a number of experiments have obtained indirect information on ripple formation from secondary ion yield changes. These have not been included in the table.

after a crossover time has been reached. Furthermore, the experiments indicate that the crossover time scales with the temperature induced surface diffusion constant.

Surface chemistry and other morphological features: While a number of attempts have been made to explain ripple formation based on chemical effects, such as $\mathrm{O}_{2}^{+}$variations [18,21,22, most of these studies were contradicted by subsequent investigations 23] where such chemical component were not present. Furthermore, in Refs. [8 10] it was unambiguously shown that the process of ripple formation is not caused by defects or inherited irregularities on the surface, but is determined merely by the primary ion characteristics. These results indicate that ripple formation is independent of microscopic details and the surface chemistry.

Ripple formation on crystalline and metallic surfaces: As the discussed experimental results have indicated, ripple formation takes place under a variety of conditions and on surfaces of different materials, including both crystalline and amorphous materials. Despite the fact that Sigmund's theory, the basis of all theories of ripple formation, has been developed for amorphous targets, it 
is worth noting that these approaches describe many features of ripple formation on crystalline surfaces as well. However, when discussing ripple formation on crystalline materials, we always have to be aware that additional effects, induced by the crystalline anisotropy, could be present. An example of ripple development on crystalline materials has been obtained for $\mathrm{Ag}(110)$ surfaces under low energy $(\epsilon \geq 800 \mathrm{eV}) \mathrm{Ar}^{+}$primary beam bombardment by Rusponi et al. 24. Ripples with wavelength of approximately $600 \AA$, oriented along the $\langle 110\rangle$ crystallographic direction, appeared in a temperature range $270^{\circ}$ $\mathrm{K} \leq T \leq 320^{\circ} \mathrm{K}$ at normal ion incidence. The ripple structure was found to be unstable at room temperature, i.e. substantial smoothing of the surface with time takes place. The structure depends on the ion flux and ion energy. Similar results are available for ion-sputtered $\mathrm{Cu}(110)$ monocrystals using a $1 \mathrm{keV} \mathrm{Ar}^{+}$ion beam [25]. For normal incidence a well defined ripple structure was observed with wave vectors whose direction changes from $\langle 001\rangle$ to $\langle 110\rangle$ as the temperature of the substrate is raised. Off-normal sputtering generated ripples whose orientation depends both on the ion direction and the surface orientation. The authors suggested that this phenomenon can be explained in terms of anisotropic surface diffusion.

Summary: As the presented results indicate, ripple formation on ion-sputtered surfaces has been observed by many groups in various systems (for a partial summary see Table (1). The main experimental results, common to most studied materials, can be summarized as follows:

- Off-normal ion bombardment often produces periodically modulated structures (ripples) on the surfaces of amorphous and crystalline materials. The wavelength of the ripples $\ell$ is usually of the order of tenths of micrometers.

- For non metallic substrates, the orientation of the ripples depends on the angle of incidence $\theta$, and in most cases is either parallel or perpendicular to the direction of the incoming ions.

- At low temperatures the ripple wavelength is independent of $T$, while it follows the Arrhenius law $\ell \sim$ $\left(1 / T^{1 / 2}\right) \exp \left(-\Delta E / k_{B} T\right)$ at higher temperatures.

- Numerous experiments find that the ripple wavelength is proportional to the ion range, and thus to the ion energy for intermediate energies.

- The ripple wavelength in many cases is independent of the ion flux, but systematic flux dependence has also been reported.

- The amplitude of the periodic modulations grows exponentially for early times, but saturates after a typical crossover time has been reached. In many instances, the ripple wavelength $\ell$ is found to be independent of time.

- Evidence for ripple formation was obtained for different materials and different primary ions, suggesting that the mechanism responsible for ripple formation is largely independent of surface chemistry, chemical reactions on the surface, or defects.

\section{B. Kinetic roughening}

Motivated by the advances in characterizing the morphology of rough surfaces, recently a number of experimental studies have focused on the scaling properties of surfaces eroded by ion bombardment 28 36. These experiments have demonstrated that under certain ion bombardment conditions ripples do not form, and the surface undergoes kinetic roughening. The goal of the present section is to review the pertinent experimental results, aiming to summarize the key features that a comprehensive theory should address.

Surface roughness and dynamical exponents: In the experiments of Eklund et al. [28,29] pyrolytic graphite was bombarded by $5 \mathrm{keV}$ Ar ions, at an angle of incidence of $60^{\circ}$. The experiments were carried out for two flux values, $6.9 \times 10^{13}$ and $3.5 \times 10^{14}$ ions s $\mathrm{sm}^{-1}$, the total fluences being $10^{16}, 10^{17}$ and $10^{18}$ ions $\cdot \mathrm{cm}^{-2}$. STM micrographs indicated that large scale features develop with continuous bombardment, the interface becoming highly correlated and rough. The scaling properties have also been probed using the Fourier transform of the heightheight correlation function, obtaining a dynamic exponent $z$ in the range $1.6-1.8$, and a roughness exponent in the range $0.2-0.4$. These exponents are consistent with the predictions of the continuum theory, describing kinetic roughening, proposed by Kardar, Parisi and Zhang (KPZ) [42, that predicts $z \simeq 1.6$ and $\alpha \simeq 0.38$ (see section IV A 1).

A somewhat larger roughness exponent has been measured for samples of iron bombarded with $5 \mathrm{keV} \mathrm{Ar}$ ions at an angle of incidence of $25^{\circ}$ [30]. The interface morphology was observed using STM, and the heightheight correlation function indicated a roughness exponent $\alpha=0.53 \pm 0.02$ 30]. The mechanism leading to such a roughness exponent is not yet understood in terms of continuum theories, since for two dimensional surfaces the existing continuum theories predict $\alpha$ values of 0.38 , $2 / 3$ and 1 [37], far from the observed roughness exponent.

Anomalous dynamic-scaling behavior of sputtered surfaces was reported by Yang et al. 31. The experiments performed on $\mathrm{Si}(111)$ surfaces with $0.5 \mathrm{keV} \mathrm{Ar}^{+}$ ions with flux $0.2 \mu \mathrm{A} / \mathrm{mm}^{2}$ in a wide range of substrate temperatures have provided evidence of scaling behavior in the limit of small distances $r$. The height-height correlation function has been found to follow $C(r)=$ $\left\langle\left(h\left(r_{o}\right)-h\left(r+r_{o}\right)\right)^{2}\right\rangle \sim r^{2 \alpha} \log t$, with $\alpha \simeq 1.15 \pm 0.08$ for temperatures lower than $530^{\circ} \mathrm{C}$. No roughening was observed for higher temperatures, demonstrating the temperature dependence of kinetic roughening.

Temperature dependence: The effect of surface relaxation due to surface diffusion on roughening of $\operatorname{GaAs}(110)$ surfaces eroded by $2 \mathrm{keV} \mathrm{Ar}^{+}$and $\mathrm{Xe}^{+}$was reported by Wang et al. [32]. They found that both the heightheight correlation function and the small scale roughness increase significantly faster during erosion at higher 
temperatures than at lower ones. The surface width in these experiments increased with $\beta=0.3$ at $T=725$ $\mathrm{K}$ and there was no evidence of scaling for lower temperatures, such as $T=625 \mathrm{~K}$. The roughness exponent has been determined as $\alpha=0.38 \pm 0.03$. In general, Ref. 31] concludes that on large scales the surfaces are rougher at higher temperatures, contrary to the expectation of smaller roughness due to increased relaxation by surface diffusion. Similar conclusions on the temperature dependence of the scaling properties were drawn in Ref. [33. A sharp transition between scaling regimes in ionbombardment of Ge(001) surfaces with $1 \mathrm{keV}$ Xe ions was observed at $T_{c}=488 \mathrm{~K}$. The regimes above and below $T_{c}$ are characterized by dynamic scaling exponents $\beta$ with values 0.4 and 0.1 , respectively. The surface roughness of $\mathrm{Si}(111)$ during low-energy (500 eV) ion bombardment at $T=610 \mathrm{~K}$ was studied in Ref. [34 using STM. It was found that the rough morphology is consistent with the early time behavior of the noisy Kuramoto-Sivashinsky (KS) equation (see Sect. IV A 3). The measured roughness exponent was $\alpha=0.7$ and the dynamic exponent was $\beta=0.25$.

Low energy ion bombardment: Recently a number of experiments and simulations have focused on low energy ion bombardment (i.e., at energies 50-500 eV), for which the secondary ion yields are smaller than one 4349 . In this systems, the effect of the ions is limited to the surface of the material, the collective effect created by the collision cascade being less relevant. Often, such low energy sputtering leads to layer-by-layer erosion, almost mirroring layer-by-layer growth phenomena. The effect of vacancy diffusion and Schwoebel barriers can be rather well studied in these systems, that include $\mathrm{Ge}(001)$ surface etching, by $240 \mathrm{eV}$ Xe ions 43,46], and $\mathrm{Si}(111)$ surfaces etched by $100 \mathrm{eV} \mathrm{Ar}$ ions [47. In the absence of the collision cascade, ripple formation and kinetic roughening seen at higher energies, the subject of this paper, do not appear.

Various experimental results on ion-bombardment induced surface roughening are summarized in Table II. These experiments demonstrate that kinetic roughening is one of the major experimental morphologies generated by ion bombardment. However, as Table III indicates, there is a considerable scattering in the scaling exponents. This scattering is not too disturbing at this point: accurate determination of the scaling exponents from experimental data is rather difficult, since often the scaling regime is masked by strong crossover effects. As we demonstrate later, due to the separation of the linear and nonlinear regimes, such crossovers are, indeed, expected in sputter erosion. Thus the main conclusion we would like to extract from this section is that numerous experiments do observe kinetic roughening, and find that scaling concepts can successfully characterize the surface morphology. It will be a major aim of the theory proposed here to account for the origin of kinetic roughening and predict the scaling exponents.

\begin{tabular}{|c|c|c|c|c|c|c|}
\hline \hline $\begin{array}{c}\text { Surface } \\
\text { material }\end{array}$ & $\begin{array}{c}\text { Ion } \\
\text { type }\end{array}$ & $\begin{array}{c}\text { Ion } \\
\text { energy } \\
\text { (keV) }\end{array}$ & $\begin{array}{c}\text { Angle } \\
\text { of } \\
\text { incidence }\end{array}$ & $\alpha$ & $\beta$ & Ref. \\
\hline \hline Graphite & $\mathrm{Ar}^{+}$ & 5 & $60^{\circ}$ & $0.2-0.4$ & $2.5-2.9$ & $\mid 28]$ \\
\hline Iron & $\mathrm{Ar}^{+}$ & 5 & $25^{\circ}$ & 0.53 & -- & 30 \\
\hline $\mathrm{Si}(111)$ & $\mathrm{Ar}$ & 0.5 & $0^{\circ}$ & $1.15^{*}$ & -- & 31 \\
\hline $\mathrm{Si}(111)$ & $\mathrm{Ar}^{+}$ & 0.5 & $0^{\circ}$ & 0.7 & 0.25 & 34 \\
\hline $\mathrm{GaAs}(110)$ & $\mathrm{Ar}^{+}$ & 2 & $0^{\circ}$ & $0.38(3)$ & 0.3 & 32 \\
\hline $\mathrm{Ge}(001)$ & $\mathrm{Xe}^{-1}$ & $30^{\circ}$ & -- & $0.1,0.4$ & 33 \\
\hline $\mathrm{Ni}, \mathrm{Cr}, \mathrm{Cu}$ & $\mathrm{Ar}^{+}$ & 1 & $86^{\circ}$ & 0.49 & -- & $36]$ \\
\hline \hline
\end{tabular}

TABLE II. Summary of the scaling exponents, characterizing the surface morphology, reported in various experiments on sputter eroded surfaces. ${ }^{*}$ - anomalous logarithmic scaling was reported in this experiment.

\section{THEORETICAL APPROACHES}

The recent theoretical studies focusing on the characterization of various surface morphologies and their time evolution have revolutionized our understanding of growth and erosion (for reviews, see 37]). The physical understanding of the processes associated with interface roughening require the use of the modern concepts of fractal geometry, universality and scaling. In Sect. IV A we review the major theoretical contributions to this area, necessary to describe the morphology of ioneroded surfaces. In Sects. IV B to IVE we then review the available theoretical approaches (whether through continuum equations or by the use of discrete atomistic models) that specifically describe surfaces eroded by ionbombardment, emphasizing the procedures which allow to describe within a continuum approach some of the relevant physical processes taking place at the surface, such as surface diffusion and beam fluctuations.

\section{A. Continuum theories of kinetic roughening}

The full strength of the continuum theories comes from the prediction of the asymptotic behavior of the growth process valid in the long time and large length scale limits. These limits are often beyond the experimentally or practically interesting time and length scales. A notable exception is sputter erosion, where both the short time ripple development and the asymptotic kinetic roughening have been observed experimentally. Consequently, next we discuss separately the continuum theories needed to understand sputter erosion. 


\section{Kardar-Parisi-Zhang (KPZ) equation}

The time evolution of a nonequilibrium interface can be described by the Kardar-Parisi-Zhang (KPZ) equation 42

$$
\frac{\partial h}{\partial t}=\nu \nabla^{2} h+\frac{\lambda}{2}(\nabla h)^{2}+\eta .
$$

The first term on the rhs describes the relaxation of the interface due to the surface tension ( $\nu$ is here a positive constant) and the second is a generic nonlinear term incorporating lateral growth or erosion. The noise, $\eta(x, y, t)$, reflects the random fluctuations in the growth process and is a set of uncorrelated random numbers with zero configurational average. For one dimensional interfaces the scaling exponents of the KPZ equation are known exactly, as $\alpha=1 / 2, \beta=1 / 3$, and $z=3 / 2$. However, for higher dimensions they are known only from numerical simulations. For the physically most relevant two dimensional surfaces we have $\alpha \simeq 0.38$ and $\beta \simeq 0.25$ [50].

If $\lambda=0$ in Eq. (5), the remaining equation describes the equilibrium fluctuations of an interface which tries to minimize its area under the influence of the external noise. This equation, first introduced by Edwards and Wilkinson (EW) [51], can be solved exactly due to its linear character, giving the scaling exponents $\alpha=(2-d) / 2$ and $\beta=(2-d) / 4$. For two dimensional interfaces $(d=2)$ we have $\alpha=\beta=0$, meaning logarithmic roughening of the interface, i.e., $w(L) \sim \log L$ for saturated interfaces, and $w(t) \sim \log t$ for early times.

\section{Anisotropic KPZ equation}

The presence of anisotropy along the substrate may drastically change the scaling properties of the KPZ equation. As a physical example consider an ion bombarded surface, where the ions arrive under oblique incidence in the $x-h$ plane. As a result, the $x$ and $y$ directions along the substrate will not be equivalent. This anisotropy is expected to appear in the erosion equation, leading to an anisotropic equation of the form $(d=2)$

$$
\begin{aligned}
\frac{\partial h}{\partial t}= & \nu_{x} \partial_{x}^{2} h+\nu_{y} \partial_{y}^{2} h+\frac{\lambda_{x}}{2}\left(\partial_{x} h\right)^{2} \\
& +\frac{\lambda_{y}}{2}\left(\partial_{y} h\right)^{2}+\eta(x, y, t),
\end{aligned}
$$

[AKPZ]

where $\partial_{x} h \equiv \partial h / \partial x$ and $\partial_{y} h \equiv \partial h / \partial y$. The anisotropy leads to surface tension and nonlinear terms that are different in the two directions, which have been incorporated in the growth equation by considering different values for the coefficients $\nu$ and $\lambda$ (in Eq. (6), $\nu_{x}$ and $\nu_{y}$ are positive constants). Equation (6) is called the anisotropic KPZ (AKPZ) equation. It was introduced by Villain [52], and its nontrivial properties were studied by Wolf 53,54. We note that if $\nu_{x}=\nu_{y}$ and $\lambda_{x}=\lambda_{y}$, Eq. (6) reduces to the KPZ equation (6). The AKPZ equation has different scaling properties depending on the signs of the coefficients $\lambda_{x}$ and $\lambda_{y}$. When $\lambda_{x} \cdot \lambda_{y}<0$, a surface described by the AKPZ equation has the same scaling properties as the EW equation. However, when $\lambda_{x} \cdot \lambda_{y}>0$ the scaling properties are described by the isotropic KPZ equation (5). Thus, changing the sign of $\lambda_{x}$ or $\lambda_{y}$ can induce morphological phase transitions from power law scaling $\left(w \sim t^{\beta} ; w(L) \sim L^{\alpha}\right)$ to logarithmic scaling $(w \sim \log t ; w(L) \sim \log L)$.

\section{Kuramoto-Sivashinsky (KS) equation}

The Kuramoto-Sivashinsky (KS) equation, originally proposed to describe chemical waves and flame fronts [55], is a deterministic equation of the form:

$$
\frac{\partial h}{\partial t}=-|\nu| \nabla^{2} h-K \nabla^{4} h+\frac{\lambda}{2}(\nabla h)^{2} \quad[K S] .
$$

While it is deterministic, its unstable and highly nonlinear character gives rise to chaotic solutions. The analysis of the KS equation for one dimensional surfaces shows 56 62 that in the limit of long time and length scales, the surface described by the KS equation is similar to that described by the KPZ equation, i.e. obeys self-affine scaling with exponents $z=3 / 2$ and $\beta=1 / 3$. The short time scale solution of KS equation reveals an unstable pattern-forming behavior, with a morphology reminiscent of ripples 56]. For two dimensional surfaces, however, the results are not clear. Computer simulations are somewhat contradictory, providing evidence for both EW and KPZ scaling [63, 64].

The anisotropic KS equation was studied in Ref. [65], indicating that for some parameter values the nonlinearities cancel each other, and lead to unstable modes dominating the asymptotic morphology. At early times the surface displays a chaotic pattern, with stable domains that nucleate and grow linearly in time until ripple domains of two different orientations are formed. The pattern of domains of perpendicularly oriented ripples coarsen with time until one orientation takes over the system.

There are various physical systems, including ion sputtering, in which the relevant equation for the surface height is a noisy version of the KS equation (77) [66, 67. Dynamical renormalization group analysis [68] for the surface dimensions $d=1$ and 2 indicate that the large distance and long time behavior of such noisy generalization of Eq. (7) is the same as that of the KPZ equation, the $d=2$ result being only quantitative.

\section{B. Bradley and Harper theory of ripple formation}

A rather successful theoretical model, capturing many features of ripple formation, was developed by Bradley 
and Harper (BH) 38. They used Sigmund's theory of sputtering 69,70 (see Sect. IVE) to relate the sputter yield to the energy deposited onto the surface by the incoming ions. This work has demonstrated for the first time that the yield variation with the local surface curvature induces an instability, which leads to the formation of periodically modulated structures. This instability is caused by the different erosion rates for troughs and crests, the former being eroded faster than the latter (see Fig. 1). Consequently, any surface perturbation increases exponentially in time. Viewing the surface profile as a smooth analytical function of coordinates, $\mathrm{BH}$ assumed that the surface can be locally approximated by a quadratic form. Due to the erosion mechanism, described in Fig. 1, the erosion rate depends on the local curvature. Combining the curvature dependent erosion velocity with the surface smoothing mechanism due to surface diffusion (see [72,73 and next section), BH derived a linear equation for surface morphology evolution

$$
\frac{\partial h}{\partial t}=-v(\theta)+\nu_{x}(\theta) \partial_{x}^{2} h+\nu_{y}(\theta) \partial_{y}^{2} h-K \nabla^{4} h .
$$

Here $\nu_{x}(\theta), \nu_{y}(\theta)$ are the effective surface tensions generated by the erosion process, dependent on the angle of incidence of the ions, $\theta, K$ is the relaxation rate due to surface diffusion $\left(K=D_{s} \gamma \Omega^{2} n / k_{B} T \exp \left\{\frac{-\Delta E}{k_{B} T}\right\}\right.$, where $\Delta E$ is the activation energy for surface diffusion, $\gamma$ is the surface free energy per unit area, $T$ is temperature, $D_{s}$ is the surface diffusion constant, $\Omega$ is the atomic volume and $n$ is the number of molecules per unit area on the surface). The physical instability illustrated in Fig. 1 leads to the negative signs of the $\nu_{x}, \nu_{y}$ coefficients in Eq. (8). Eq. (8) is linearly unstable, with a Fourier mode $k_{c}$ whose amplitude exponentially dominates all the others. This mode is observed as the periodic ripple structure. Using linear stability analysis, BH derived from Eq. (8) the ripple wavelength as

$$
\ell_{c}=2 \pi / k_{c}=2 \pi \sqrt{\frac{2 K}{|\nu|}} \sim(J T)^{-1 / 2} \exp \left\{\frac{-\Delta E}{k_{B} T}\right\}
$$

where $\nu$ is the largest in absolute value of the two negative surface tension coefficients, $\nu_{x}$ and $\nu_{y}$, and $J$ is the ion flux. The calculation also predicts that the ripple direction is a function of the angle of incidence: for small $\theta$ the ripples are parallel to the ion direction, while for large $\theta$ they are perpendicular to it. As subsequent experiments have demonstrated [15,18], the $\mathrm{BH}$ model predicts well the ripple wavelength and orientation. On the other hand, the $\mathrm{BH}$ equation (8) is linear, predicting unbounded exponential growth of the ripple amplitude, thus it cannot account for the stabilization of the ripples and for kinetic roughening, both phenomena being strongly supported by experiments (see Sect. III A III B).

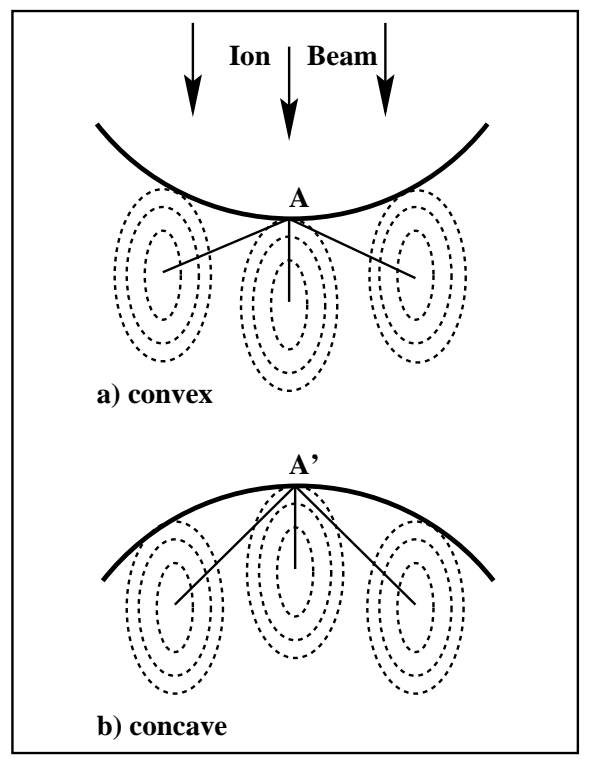

FIG. 1. Schematic illustration of the physical origin of the instability during ion erosion of nonplanar surfaces. A surface element with convex geometry (a) is eroded faster than that with a concave geometry (b), due to the smaller distances (solid lines) the energy has to travel from the impact point to the surface (A or A' points).

Furthermore, the BH model cannot account for low temperature ripple formation since the only smoothing mechanism it considers is of thermal origin. At low temperatures the ion energy and flux dependence of the ripple wavelength also disagree with the $\mathrm{BH}$ predictions. Despite these shortcomings, the $\mathrm{BH}$ theory represents a major step in understanding the mechanism of surface evolution in ion sputtering since for the first time it uncovered the origin of the ion induced surface instability. Recently a generalization of $\mathrm{BH}$ linear theory has been successfully introduced [71] to account for the thermally activated anisotropic surface diffusion present in metallic substrates such as $\mathrm{Cu}(110)$.

\section{Surface diffusion and deposition noise}

At high temperatures surface diffusion and fluctuations in the ion beam flux are relevant physical mechanisms taking place on the surface 74. In this section, we discuss the standard approach to include these phenomena in continuum models. Let us consider the simplest scenario: atoms are deposited on a surface, whereupon they diffuse. If we assume that surface diffusion is the only relaxation mechanism present, the height $h$ obeys a continuity equation of the form

$$
\frac{\partial h}{\partial t}+\tilde{\nabla} \cdot \boldsymbol{j}=0
$$

where $\boldsymbol{j}$ is a surface current density tangent to the surface, and $\tilde{\nabla}$ is calculated in a frame with axes parallel to 
the surface 75]. In general, $\boldsymbol{j}$ is given by the gradient of a chemical potential $\mu$,

$$
\boldsymbol{j} \propto-\tilde{\nabla} \mu(\boldsymbol{r}, t) \equiv-\tilde{\nabla}^{2} \frac{\delta \mathcal{F}[h]}{\delta h},
$$

where $\mu$ minimizes the free energy functional of the surface $\mathcal{F}[h]$ and $\tilde{\nabla}^{2}$ is the surface Laplacian or the LaplaceBeltrami operator. Taking the latter to be proportional to the total surface area

$$
\mathcal{F}[h]=\int d \boldsymbol{r} \sqrt{g},
$$

with $g$ as defined in Appendix A, and neglecting third or higher powers of derivatives of $h$ one arrives [73] at

$$
\frac{\partial h}{\partial t}=-K \nabla^{2}\left(\nabla^{2} h\right) \equiv-K \nabla^{4} h,
$$

where $K$ is a positive constant. Eq. (13) is the socalled linear MBE equation [37]. For an amorphous solid in equilibrium with its vapor Eq. (13) was obtained in 72, 73, together with the expression for the coefficient $K$ as in Eq. (8).

In addition to the deterministic processes, there is considerable randomness in sputter erosion due to fluctuations in the intensity of the ion beam. The ion flux is defined as the number of particles arriving on the unit surface (or per lattice site) in unit time. At large length scales the beam flux is homogeneous with an average intensity $J$, but there are local random fluctuations, $\eta(\mathbf{x}, t) \equiv \delta J(\mathbf{x}, t)$, uncorrelated in space and time. We can include fluctuations in Eq. (13) by considering the ion flux to be the sum of the average flux $J$ and the noise $\eta$, which has zero average,

$$
\langle\eta(\mathbf{x}, t)\rangle=0
$$

and is uncorrelated,

$$
\left\langle\eta(\mathbf{x}, t) \eta\left(\mathbf{x}^{\prime}, t^{\prime}\right)\right\rangle=J \delta\left(\mathbf{x}-\mathbf{x}^{\prime}\right) \delta\left(t-t^{\prime}\right),
$$

where we have assumed a Poisson distribution for the shot noise. Consequently, the stochastic growth equation describing surface diffusion and fluctuations in an erosion process has the form

$$
\frac{\partial h}{\partial t}=-K \nabla^{4} h-J+\eta(\mathbf{x}, t) .
$$

This variant of Eq. (13) was introduced independently by Wolf and Villain [76], and by Das Sarma and Tamborenea [77, and played a leading role in developing our understanding of MBE. We will use the methods leading to (16) to incorporate the smoothing by surface diffusion in our model of ion erosion. Note, however, that as numerous experimental studies 78 83] indicate, ion bombardment leads to an enhancement of the surface adatom mobility and thus may drastically change the relaxation mechanism, as compared to regular surface diffusion.

\section{Microscopic models of ripple formation and roughening}

Computer simulations provide invaluable insight into microscopic processes taking place in physical systems. Consequently, a number of recent studies have focused on modeling ripple formation at the microscopic level. These studies have proven useful in resolving issues related to low temperature ripple formation and provided important ideas regarding the physical mechanism governing ripple formation [84 93]. Here we shortly discuss the conclusions reached in some of the most representative numerical work.

Monte Carlo simulations of sputter-induced roughening were reported by Koponen et al. 855 90]. Roughening of amorphous carbon surfaces bombarded by $5 \mathrm{keV} \mathrm{Ar}^{+}$ ions was studied in 85 87 for incidence angles between $0^{\circ}$ and $60^{\circ}$. It was found that ion bombardment induces self-affine topography on the submicrometer scale, the roughness exponent being $\alpha \simeq 0.25-0.47$ depending on the angle of incidence 86, 87. The growth exponent $\beta$ was found to be strongly dependent on the relaxation mechanism used and changed from $\beta \simeq 0.3$ in the model without relaxation to $\beta \simeq 0.2-0.14$ when different relaxation rules were used in the simulations. At the same time the roughness exponent $\alpha$ was found to be relatively insensitive to the relaxation process on the nanometer scales. Analogous results were obtained for $\mathrm{C}$ ions [89]. In this Reference, the ripple wavelength was found to be relatively independent of the ion energy or the magnitude of surface diffusion. Ripple formation was observed even at zero temperature, when surface diffusion was switched off, indicating the presence of ion induced smoothing. Furthermore, these simulations led to the observation of traveling ripples, as predicted by continuum theories (see section VI A 1). Similarly, for $5 \mathrm{keV} \mathrm{Ar}^{+}$bombardment of amorphous carbon substrates, the ripple wave vector is seen [90] to change from parallel to normal to the beam direction as the incidence angle is increased, in agreement with BH linear theory, (see Sect. IV). The ripple structure was again observed even when no explicit relaxation mechanism was incorporated in the simulations, and ripple travelling also occurs. For length scales comparable to the cascade dimensions, self-affine topography is observed.

A discrete stochastic model was introduced in Ref. 91.92 to study the morphological evolution of amorphous one dimensional surfaces under ion-bombardment. This is a solid-on-solid model incorporating the erosion rate dependence of surface curvature, the local slope dependence of the sputtering yield, and thermally activated surface diffusion. Up to four different dynamical regimes have been identified. Initially the surface relaxes by surface diffusion with a growth exponent $\beta \simeq 0.38$, until the onset of the linear BH instability. The instability induces rapid growth $(\beta>0.5)$. In this regime the local 
slopes increase rapidly, which triggers non-linear effects eventually stabilizing the surface, $\beta$ taking up the EW value $\beta \simeq 1 / 4$, which indicates that an effective positive value of the surface tension has been generated. Finally, in the asymptotic time limit $\beta$ reaches the KPZ value $\beta \simeq 1 / 3$. This behavior is consistent with that displayed by the noisy KS equation [68]. Furthermore, the analytical study 94 using the master equation approach to interface models [95] has shown that the noisy KS equation indeed provides the continuum limit of the discrete stochastic model of Ref. [91]. Conversely, the results of the simulations in 91] support the theoretical conclusions of Ref. 68] that the asymptotic behavior of the noisy KS equation is the same as that of the KPZ equation for one and two dimensional surfaces.

In summary, Monte Carlo simulations of the sputtering process of amorphous materials have shown that intermediate and high energy ion bombardment may lead to ripple formation in a wide parameter range. Furthermore, ripple formation was observed even at zero temperature. Computer simulations have also confirmed the linear dependence of the ripple wavelength on the incident ion penetration depth and the fact that ripple formation is a process fully determined by the incident ion characteristics and not caused by any defects, irregularities or surface chemistry. The same simulations have confirmed that under some bombarding conditions the surface is rough, and obeys scaling.

\section{E. Sigmund's theory of sputtering}

The erosion rate of ion bombarded surfaces is characterized by the sputtering yield, $Y$, defined as the average number of atoms leaving the surface of a solid per incident particle. In order to calculate the yield and to predict the surface morphology generated by ion bombardment, we first need to understand the mechanism of sputtering, resulting from the interaction of the incident ions and the substrate [1, 3]. In the process of sputtering the incoming ions penetrate the surface and transfer their kinetic energy to the atoms of the substrate by inducing cascades of collisions among the substrate atoms, or through other processes such as electronic excitations. Whereas most of the sputtered atoms are located at the surface, the scattering events that might lead to sputtering take place within a certain layer of average depth $a$, which is the average penetration depth of the incident ion. A qualitative picture of the sputtering process is as follows: an incoming ion penetrates into the bulk of the material and undergoes a series of collisions with the atoms of the substrate. Some of the atoms undergo secondary collisions, thereby generating another generation of recoiling atoms. A vast majority of atoms will not gain enough energy to leave their lattice positions permanently. However, some of them will be permanently removed from their sites.

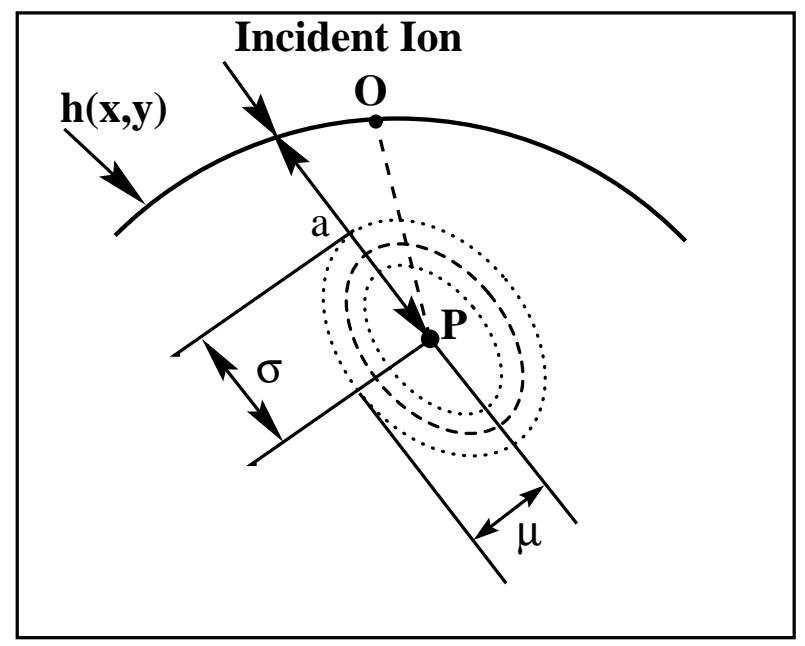

FIG. 2. Schematic illustration of the energy distributed by an incident ion. While the collision process induced by a ion is rather complex, according to Sigmund it can be reduced to the following effective process: The ion penetrates the bulk of the material and stops at point $P$, where all its kinetic energy is released and spread out to the neighboring sites following a Gaussian form with widths $\sigma$ and $\mu$.

The atoms located in the close vicinity of the surface, which can gain enough energy to break their bonds, will be sputtered. Usually the number of sputtered atoms is orders of magnitude smaller than the total number of atoms participating in the collision cascade.

A quantitative description of the process of ion sputtering was developed by Sigmund 69]. Assuming an amorphous target, Sigmund derived a set of transport equations describing the energy transfer during the sputtering process. A practically important result of Sigmund's theory is the prediction of the deposited energy distribution: the ion deposited at a point $P$ inside the bulk of the material spreads its kinetic energy according to the Gaussian distribution

$$
E\left(\boldsymbol{r}^{\prime}\right)=\frac{\epsilon}{(2 \pi)^{3 / 2} \sigma \mu^{2}} \exp \left\{-\frac{Z^{\prime 2}}{2 \sigma^{2}}-\frac{X^{\prime 2}+Y^{\prime 2}}{2 \mu^{2}}\right\} .
$$

In (17) $Z^{\prime}$ is the distance from point $P$ to point $O$ measured along the ion trajectory, and $X^{\prime}, Y^{\prime}$ are measured in the plane perpendicular to it (see Fig. 2 and the inset of Fig. 5); $\epsilon$ denotes the total energy carried by the ion and $\sigma$ and $\mu$ are the widths of the distribution in directions parallel and perpendicular to the incoming beam respectively. Deviations of the energy distribution from Gaussian (17) occur mainly when $M_{1}>M_{2}$, where $M_{1}$ is the mass of the projectile and $M_{2}$ is the mass of the target atom. As shown by Sigmund [69,70] and Winterbom [96], electronic stopping doesn't affect much the shape of deposited-energy distribution. Subsequently, Monte Carlo simulations of the sputtering process have demonstrated that the deposited-energy distribution and damage distribution can be well approximated by Gaussian 
for intermediate and high energies. In general, comparison of Sigmund's theory with experimental results has shown that it describes well the qualitative behavior of the backsputtering yield, and in many cases there is good quantitative agreement as well [1] 3.

A quantity of central importance is the mean path length of an ion traveling inside the bulk of the material (see Fig. 2), often called penetration depth, given by

$$
a(\epsilon)=\frac{1-m}{2 m} \gamma^{m-1} \frac{\epsilon^{2 m}}{N C_{m}}
$$

where $N$ is the target atom density, $C_{m}$ is a constant dependent on the parameters of the interatomic potential [70] and the exponent $m=m(\epsilon)$ varies slowly from $m=1$ at high energies to $m \simeq 0$ at very low energies. In the region of intermediate energies, i.e. for $\epsilon$ between 10 and $100 \mathrm{keV}, m \simeq 1 / 2$ and we can approximate the penetration depth as $a(\epsilon) \sim \epsilon$.

Eq. (17) describes the effect of a single incident ion. Actually, the sample is subject to an uniform flux $J$ of bombarding ions, penetrating the solid at different points simultaneously, such that the erosion velocity at an arbitrary point $O$ depends on the total power $\mathcal{E}_{O}$ contributed by all the ions deposited within the range of the distribution (17). If we ignore shadowing effects and redeposition of the eroded material, the normal erosion velocity at $O$ is given by

$$
V_{O}=p \int_{\mathcal{R}} d \boldsymbol{r} \Phi(\boldsymbol{r}) E(\boldsymbol{r}),
$$

where the integral is taken over the region $\mathcal{R}$ of all points at which the deposited energy contributes to $\mathcal{E}_{O}, \Phi(\boldsymbol{r})$ is a local correction to the uniform flux $J$ due to variation of the local slopes, and the material constant $p$ depends on the surface binding energy and scattering cross-section 69,70 as

$$
p=\frac{3}{4 \pi^{2}} \frac{1}{N U_{o} C_{o}},
$$

where $U_{o}$ is the surface binding energy and $C_{o}$ is a constant proportional to the square of the effective radius of the interatomic interaction potential.

While the predictions of Sigmund's theory have been checked on many occasions, it also has well known limitations. Next we list two, that will limit our theory on the surface morphology as well: (a) the assumption of random slowing down and arbitrary collisions works satisfactorily only at intermediate and high energies, i.e. when $\epsilon \sim 1-100 \mathrm{keV}$, but may break down at low energies; (b) the assumption of a planar surface may influence the magnitude of the yield, since surface roughness has a tendency to increase the yield 97, 98.

\section{CONTINUUM EQUATION FOR THE SURFACE HEIGHT}

Sigmund's theory, while offering a detailed description of ion bombardment, is not able to provide direct information about the morphology of ion-sputtered surfaces. While Eq. (19) provides the erosion velocity, in the present form it cannot be used to make analytical predictions regarding the dynamical properties of surface evolution. To achieve such a predictive power, we have to eliminate the nonlocality contained in the integral (19) and derive a continuum equation describing the surface evolution depending only on the local surface morphology. The main goal of this section is to provide a detailed derivation of such an equation starting from Eq. (19). The properties of the obtained equation will be discussed in the following sections.

We start by summarizing the main steps that we follow in the derivation of the equation for the surface morphology evolution:

(i) Using Eq. (19), we calculate the normal component of the erosion velocity $V_{O}$ at a generic point $O$ of the surface. This calculation can be performed in a local frame of reference $(\hat{X}, \hat{Y}, \hat{Z})$, defined as follows: the $\hat{Z}$ axis is chosen to be parallel to the local normal to the surface at point $O$. The $\hat{Z}$ axis forms a plane with the trajectory of an ion penetrating the surface at $O$. We choose the $\hat{X}$ axis to lie in that plane and be perpendicular to $\hat{Z}$. Finally, $\hat{Y}$ is perpendicular to the $(\hat{X}, \hat{Z})$ plane and completes the local reference frame, as shown in Fig. 3 .

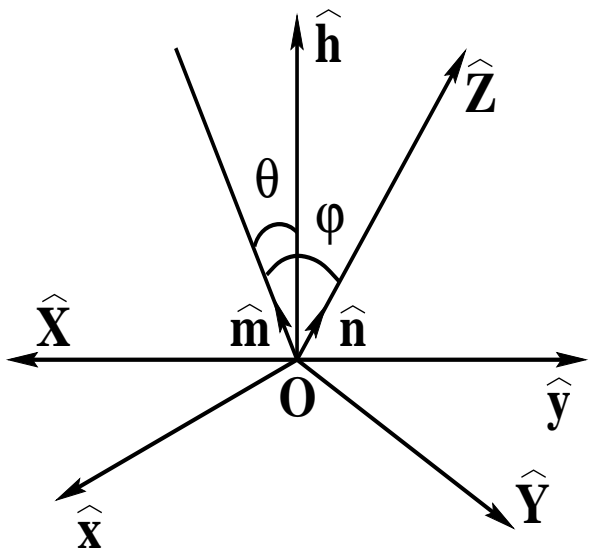

FIG. 3. Illustration the local reference frame $(\hat{X}, \hat{Y}, \hat{Z})$. The $\hat{Z}$ axis is parallel to the local normal to the surface $\hat{n}$. The ions arrive to the surface along $-\hat{m}$. The $\hat{X}$ axis is in the plane defined by $\hat{Z}$ and $\hat{m}$, while the $\hat{Y}$ axis is perpendicular to this plane. The laboratory coordinate frame $(\hat{x}, \hat{y}, \hat{h})$ has its $\hat{h}$ axis perpendicular to the flat substrate, $\hat{h}$ and $\hat{m}$ define the $(\hat{x}, \hat{h})$ plane and $\hat{y}$ is perpendicular to it. The incidence angle measured in the local reference frame is $\varphi$, and $\theta$ in the laboratory frame. 
(ii) Next we relate the quantities measured in the local frame $(\hat{X}, \hat{Y}, \hat{Z})$ to those measured in the laboratory frame $(\hat{x}, \hat{y}, \hat{h})$. The latter is defined by the experimental configuration as follows: $\hat{h}$ is the direction normal to the uneroded flat surface. The ion direction together with the $\hat{h}$ axis define the $(\hat{x}, \hat{h})$ plane. Finally, the $\hat{y}$ axis is perpendicular to the $(\hat{x}, \hat{h})$ plane (see Fig. 3 and Appendix A). Furthermore, we have to take into account the fact that the local angle of incidence $\varphi$, which is the angle between the ion trajectory and the local normal to the surface, changes from point to point along the surface. Consequently, $\varphi$ is a function of the local value of the slope at $O$ (as measured in the laboratory frame), and the angle $\theta$ between the ion trajectory and the normal $\hat{n}$ to the uneroded surface.

(iii) Finally, to obtain the equation of motion for the surface profile $h(x, y, t)$, we have to project the normal component of the velocity of erosion onto the global $\hat{h}$ axis. The time derivative of $h(x, y, t)$ at any point $O$ on the surface is proportional to the surface erosion velocity $V_{O}$ at that point and the local normal is defined by the gradient of the surface profile $h(x, y, t)$ at $O$.

Having defined our objectives and outlined the strategy, we move on to the description of the calculations. We consider point $O$ to be the origin of the local system of coordinates $(\hat{X}, \hat{Y}, \hat{Z})$. To describe the surface profile in a neighborhood of $O$ we assume that the surface can be described in terms of a smooth analytical infinitely differentiable function, i.e. there are no singularities and overhangs, and thus we can approximate the surface profile at an arbitrary point $(X, Y, Z)$ by 99

$$
\begin{aligned}
Z(X, Y) & \simeq \frac{\Delta_{20} X^{2}}{a}+\frac{\Delta_{02} Y^{2}}{a}+ \\
& +\sum_{m, n=0, n+m=3,4}^{4} \frac{\Delta_{n m}}{a^{m+n-1}} X^{n} Y^{m}
\end{aligned}
$$

where, for later convenience, we introduced the following notations:

$$
\Delta_{n m}=\frac{a^{n+m-1}}{n ! m !} \frac{\partial^{n+m} Z(X, Y)}{\partial^{n} X \partial^{m} Y} .
$$

Here $\Delta_{20}$ and $\Delta_{02}$ are proportional to the principal curvatures of the surface, i.e., to the inverses of the principal radii of curvature, $R_{X}$ and $R_{Y}$. It must be noted that, in our approximation, $\hat{X}$ and $\hat{Y}$ (see Fig. 3 ) are the two principal directions of the surface at $O$, along which the curvatures are extremal. This implies the absence in Eq. (21) of cross-terms of the type $\sim X Y$, i.e., we neglected the term $\partial^{2} Z(X, Y) / \partial X \partial Y$ at $O$.

Due to its exponential nature, the deposited energy distribution (17) decays very fast and, consequently, only particles striking the surface at a point $(X, Y, Z)$ such that $X / a, Y / a$ are of order unity, contribute nonnegligibly to the energy reaching the surface at $O$. We further assume that the surface varies slowly enough so that $R_{X}, R_{Y}$ and the inverses of the higher order derivatives are much larger than the penetration depth $a$, i.e. the surface is smooth on length scales close to $a$ (this fact is supported by nearly all experimental results). Now we can calculate the various factors appearing in the integral (19).

To proceed with Eq. (19) we note that, with respect to local surface orientation, only the normal component of the incident flux contributes to ion erosion. Figure 4 1 illustrates the calculation of the normal component of the flux. In the figure we consider a point at the surface $(X, Y, Z)$, and two other points also on the surface, at infinitesimal distances $L$ and $N$ away from the former. We can estimate the correction to the average flux $J$ due to the surface slopes by projecting a square perpendicular to the ion beam with area $n \times l$ onto the surface area element intersected by the ion trajectories. The result is

$$
\Phi(\boldsymbol{r}) \simeq J \frac{l n}{L N},
$$

where $J$ is the average flux. From Fig. 1 .

$$
\Delta \varphi=\tan ^{-1}\left(\frac{\partial Z}{\partial X}\right) \simeq \frac{\partial Z}{\partial X}
$$

and

$$
\frac{\ell}{L}=\cos (\varphi+\Delta \varphi) \simeq \cos \varphi-\frac{\partial Z}{\partial X} \sin \varphi .
$$

On the other hand, we also have (see Fig. 目)

$$
\frac{n}{N}=\cos \alpha \simeq 1-\frac{1}{2}\left(\frac{\partial Z}{\partial Y}\right)^{2} \simeq 1,
$$

so that, combining Eqs. (23)-(26), and neglecting powers of derivatives of the height, we obtain the correction to the flux

$$
\Phi(\boldsymbol{r}) \simeq J\left(\cos \varphi+\left(\partial_{X} Z\right) \sin \varphi\right) .
$$

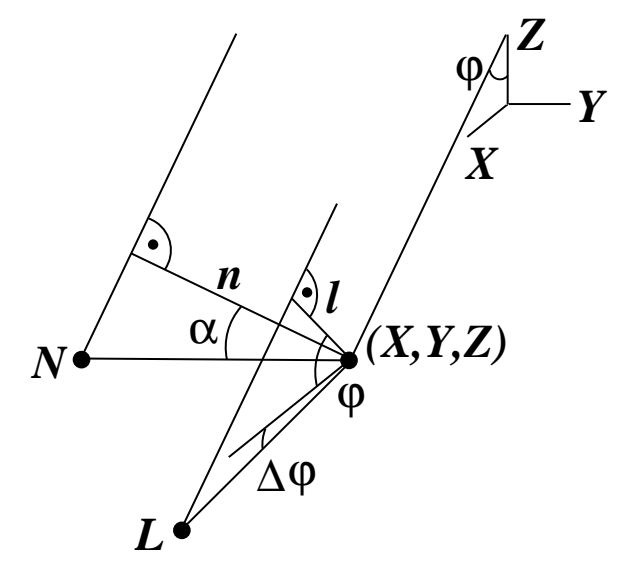

FIG. 4. Illustration of the calculation of the local correction to the average flux $J$ due to the surface curvature. 


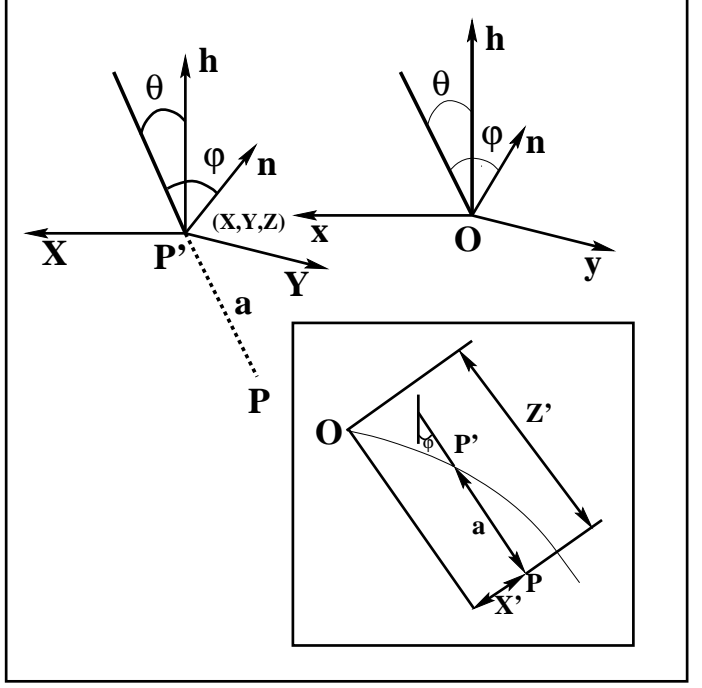

FIG. 5. Reference frame for the calculation of the erosion velocity at point $O$. Following a straight trajectory (thick solid line) the ion penetrates an average distance $a$ inside the solid (dotted line) after which it completely spreads out its kinetic energy. The energy released at point $P$ contributes to erosion velocity at $O$. Inset shows the lateral view for $Y^{\prime}=0$.

Within the same approximation, the surface element $d \mathbf{r}$ in Eq. (19) can be obtained in the local coordinate system $(\hat{X}, \hat{Y}, \hat{Z})$ as

$$
d \mathbf{r} \simeq d X d Y
$$

Next we determine the distances $X^{\prime}, Y^{\prime}, Z^{\prime}$ appearing in the exponential distribution (17), evaluating them in the local coordinate system. Using Fig. 5, we have

$$
\begin{aligned}
& X^{\prime}=X \cos \varphi+Z \sin \varphi, \\
& Y^{\prime}=Y, \\
& Z^{\prime}=a+X \sin \varphi-Z \cos \varphi .
\end{aligned}
$$

Using these expressions, the correction to the ion flux (27), the deposited energy distribution (17) and the expression for the surface area element $d \mathbf{r}(28)$, we can calculate the integral (19) providing the erosion velocity $V_{O}$. Introducing the dimensionless variables $\zeta_{X}=X / a$, $\zeta_{Y}=Y / a$, and $\zeta_{Z}=Z / a$, and extending the integration limits to infinity, we obtain the following expression for the erosion velocity in the laboratory coordinate frame

$$
\begin{aligned}
V_{O} & =\frac{\epsilon p J a^{2}}{\sigma \mu^{2}(2 \pi)^{3 / 2}} \exp \left(-a_{\sigma}^{2} / 2\right) \\
& \times \int_{-\infty}^{\infty} \int_{-\infty}^{\infty} d \zeta_{X} d \zeta_{Y}\left(\cos \varphi+\frac{\partial \zeta_{Z}}{\partial \zeta_{X}} \sin \varphi\right) \\
& \times \exp \left(-\zeta_{Y}^{2} L^{2}\right) \exp \left(-\zeta_{X} A\right) \exp \left(-\frac{1}{2} B_{1} \zeta_{X}^{2}\right) \\
& \times \exp \left(-4 D \zeta_{Z}^{2}\right) \exp \left(-2 C \zeta_{X} \zeta_{Z}\right) \exp \left(B_{2} \zeta_{Z}\right),
\end{aligned}
$$

where we used the following notations

$$
\begin{aligned}
A & =a_{\sigma}^{2} \sin \varphi \\
B_{1} & =a_{\sigma}^{2} \sin ^{2} \varphi+a_{\mu}^{2} \cos ^{2} \varphi, \\
B_{2} & =a_{\sigma}^{2} \cos \varphi \\
C & =\frac{1}{2}\left(a_{\mu}^{2}-a_{\sigma}^{2}\right) \sin \varphi \cos \varphi, \\
D & =\frac{1}{8}\left(a_{\mu}^{2} \sin ^{2} \varphi+a_{\sigma}^{2} \cos ^{2} \varphi\right), \\
L & =\frac{a_{\mu}}{\sqrt{2}} .
\end{aligned}
$$

It must be noted that Eq. (30) coincides with the twodimensional version of the local erosion velocity derived in Ref. [38]. Now we use the approximation for the surface profile given by Eq. (21). Taking a small $\Delta_{n m}$ (see Eq. (22)) expansion of the $C$ and $B_{2}$ coefficients in Eq. (30) and evaluating the Gaussian integrals over $\zeta_{X}$ and $\zeta_{Y}$, we get

$$
\begin{aligned}
V_{O} & =\frac{\epsilon p J a^{2}}{\sigma \mu \sqrt{2 \pi}} \exp \left(-a_{\sigma}^{2} / 2\right) \exp \left\{\frac{A^{2}}{2 B_{1}}\right\} \frac{1}{\sqrt{B_{1}}} \\
& \times\left[\cos \varphi+\Gamma_{20} \Delta_{20}+\Gamma_{02} \Delta_{02}+\Gamma_{30} \Delta_{30}\right. \\
& \left.+\Gamma_{21} \Delta_{21} \Gamma_{40} \Delta_{40}+\Gamma_{22} \Delta_{22}+\Gamma_{04} \Delta_{04}\right]
\end{aligned}
$$

The expressions for the coefficients $\Gamma_{n m}$ can be found in Appendix B.

Next we have to rewrite $V_{O}$ in terms of the laboratory coordinates $(x, y, h)$, which we perform in two steps. First, we write the angle $\varphi$ as a function of $\theta$ and the slopes of the surface at $O$ as measured in the laboratory frame. Second, we perform the transformation between the local and the laboratory coordinates. For both steps we will have to make expansions in powers of derivatives of $h(x, y, t)$. In line with our earlier assumption on the smoothness of the surface, we will assume that $h$ varies smoothly enough so that we can neglect products of derivatives of $h$ for third and higher orders. In the laboratory frame, the neglect of overhangs allows us to describe a generic point at the surface, such as $O$, with coordinates $(x, y, h(x, y))$. Considering now the unit vectors $\hat{n}, \hat{m}$ shown in Fig. 3 , the angle $\varphi$ is given by

$$
\begin{aligned}
\cos \varphi & =\hat{m} \cdot \hat{n}=\frac{\cos \theta-\left(\partial_{x} h\right) \sin \theta}{\sqrt{1+\left(\partial_{x} h\right)^{2}+\left(\partial_{y} h\right)^{2}}}, \\
\sin \varphi & =\left(\sin ^{2} \theta+2\left(\partial_{x} h\right) \sin \theta \cos \theta\left(\partial_{x} h\right)^{2} \cos ^{2} \theta\right. \\
& \left.+\left(\partial_{y} h\right)^{2}\right)^{1 / 2} \times\left(1+\left(\partial_{x} h\right)^{2}+\left(\partial_{y} h\right)^{2}\right)^{-1 / 2} .
\end{aligned}
$$

Thus far, expressions (33a)-(33b) are exact, and the values of $\partial_{x} h$ and $\partial_{y} h$ are already evaluated in the laboratory frame of reference. To implement our approximations, in principle we have to separate the cases for normal $(\theta=0)$ and off-normal $(\theta \neq 0)$ incidence. Nevertheless, it can be shown that the former case can be 
obtained as a smooth limit of the latter. Therefore in the following we give the expressions pertaining to the off-normal incidence and refer the reader to Appendix $\mathbf{9}$ for details on the $\theta=0$ limit. Expanding (33a and (33b) in powers of the surface height derivatives, we obtain

$$
\begin{aligned}
\cos \varphi \simeq & \cos \theta-\left(\partial_{x} h\right) \sin \theta \\
& -\frac{1}{2}\left(\left(\partial_{x} h\right)^{2}+\left(\partial_{y} h\right)^{2}\right) \cos \theta, \\
\sin \varphi \simeq & \sin \theta+\left(\partial_{x} h\right) \cos \theta-\frac{1}{2}\left(\partial_{x} h\right)^{2} \sin \theta \\
& +\frac{1}{2}\left(\partial_{y} h\right)^{2} \frac{\cos ^{2} \theta}{\sin \theta} .
\end{aligned}
$$

Note that these expressions are invariant under the coordinate transformation $y \rightarrow-y$, but not under $x \rightarrow-x$, a consequence of $\theta$ being non-zero and of our choice of coordinates. Naturally, the $\theta \rightarrow 0$ limit restores the symmetry in the $x$ direction.

Having obtained the expressions (34a and (34b), we can return to Eq. (32) to calculate the dependence of $V_{O}$ on the slopes at $O$. Finally, all derivatives in (22) have to be expressed in terms of the laboratory coordinates. This can be accomplished given the relation between the base vectors of the local frame $(\hat{X}, \hat{Y}, \hat{Z})$ and those of the laboratory frame $(\hat{x}, \hat{y}, \hat{h})$, derived in Appendix A. If the coordinates of a generic vector $\boldsymbol{r}$ are given by

$$
\begin{aligned}
& \boldsymbol{r}=X \hat{X}+Y \hat{Y}+Z \hat{Z} \quad \text { local frame, } \\
& \boldsymbol{r}=x \hat{x}+y \hat{y}+z \hat{z} \quad \text { laboratory frame, }
\end{aligned}
$$

then these quantities are related to each other through

$$
\left(\begin{array}{l}
x \\
y \\
z
\end{array}\right)=\mathcal{M}\left(\begin{array}{l}
X \\
Y \\
Z
\end{array}\right),
$$

where $\mathcal{M}$ is a matrix which has as columns the components of the $(\hat{X}, \hat{Y}, \hat{Z})$ set of vectors in terms of the $(\hat{x}, \hat{y}, \hat{z})$ (see Appendix A). To obtain the expression for the erosion velocity, analogous to 32 , in the laboratory frame, we use Eqs. (34a), (34b), and $\mathcal{M}$ along with the chain rule for differentiation, and perform expansions in powers of derivatives of $h(x, y, t)$. After some algebra we obtain in the laboratory frame

$$
\frac{\partial^{n+m} h}{\partial^{n} X \partial^{m} Y} \simeq \frac{\partial^{n+m} h}{\partial^{n} x \partial^{m} y},
$$

up to fourth order in products of derivatives of $h(x, y)$. To summarize our results thus far, within the approximations leading to (32) and neglecting nonlinearities of cubic and higher orders in derivatives of $h$ in the laboratory frame, we obtained Eq. (37), providing the relation between the derivatives in the two reference frames, and relations (34a) and (34b) for the angle of incidence measured in the local frame as a function of the angle of incidence $\theta$ and of the surface slopes.

Finally, to relate the velocity of erosion $V_{O}$, which is normal to the surface at $O$, to the velocity of erosion of the surface along the $h$ axis, $\partial h / \partial t$, we have to project the former onto the latter, obtaining

$$
\frac{\partial h}{\partial t}=-V_{O} \sqrt{g}
$$

where the negative sign accounts for the fact that $V_{O}$ is the rate at which the surface is eroded, i.e. the average height decreases. Furthermore, taking into account surface diffusion effects, together with the fluctuations (shot noise) in the flux of the bombarding particles, as discussed in Section IVC, we complete (38) by adding these physical effects

$$
\frac{\partial h}{\partial t}=-V_{O} \sqrt{g}-K \nabla^{4} h+\eta(\boldsymbol{r}, t),
$$

Finally, we have to write down the contribution of the $-V_{O} \sqrt{g}$ term to the evolution equation (39). Performing a small slope expansion and using Eqs. (32), (34a), and (34b), we obtain

$$
\begin{aligned}
\frac{\partial h}{\partial t}= & -v_{0}+\gamma \frac{\partial h}{\partial x}+\xi_{x}\left(\frac{\partial h}{\partial x}\right)\left(\frac{\partial^{2} h}{\partial x^{2}}\right)+\xi_{y}\left(\frac{\partial h}{\partial x}\right)\left(\frac{\partial^{2} h}{\partial y^{2}}\right)+\nu_{x} \frac{\partial^{2} h}{\partial x^{2}}+\nu_{y} \frac{\partial^{2} h}{\partial y^{2}}+\Omega_{1} \frac{\partial^{3} h}{\partial x^{3}}+\Omega_{2} \frac{\partial^{3} h}{\partial x \partial y^{2}} \\
& -D_{x y} \frac{\partial^{4} h}{\partial x^{2} \partial y^{2}}-D_{x x} \frac{\partial^{4} h}{\partial x^{4}}-D_{y y} \frac{\partial^{4} h}{\partial y^{4}}-K \nabla^{4} h+\frac{\lambda_{x}}{2}\left(\frac{\partial h}{\partial x}\right)^{2}+\frac{\lambda_{y}}{2}\left(\frac{\partial h}{\partial y}\right)^{2}+\eta(x, y, t)
\end{aligned}
$$

where the coefficients are given by the expressions

$$
\begin{gathered}
v_{0}=F c \\
\gamma=F \frac{s}{f^{2}}\left\{a_{\sigma}^{2} a_{\mu}^{2} c^{2}\left(a_{\sigma}^{2}-1\right)-a_{\sigma}^{4} s^{2}\right\} \\
\nu_{x}=F a \frac{a_{\sigma}^{2}}{2 f^{3}}\left\{2 a_{\sigma}^{4} s^{4}-a_{\sigma}^{4} a_{\mu}^{2} s^{2} c^{2}+a_{\sigma}^{2} a_{\mu}^{2} s^{2} c^{2}-a_{\mu}^{4} c^{4}\right\},
\end{gathered}
$$




$$
\begin{aligned}
& \lambda_{x}=F \frac{c}{2 f^{4}}\left\{a_{\sigma}^{8} a_{\mu}^{2} s^{4}\left(3+2 c^{2}\right)+4 a_{\sigma}^{6} a_{\mu}^{4} s^{2} c^{4}-a_{\sigma}^{4} a_{\mu}^{6} c^{4}\left(1+2 s^{2}\right)\right. \\
& \left.-f^{2}\left(2 a_{\sigma}^{4} s^{2}-a_{\sigma}^{2} a_{\mu}^{2}\left(1+2 s^{2}\right)\right)-a_{\sigma}^{8} a_{\mu}^{4} s^{2} c^{2}-f^{4}\right\} \\
& \nu_{y}=-F a \frac{c^{2} a_{\sigma}^{2}}{2 f} \\
& \lambda_{y}=F \frac{c}{2 f^{2}}\left\{a_{\sigma}^{4} s^{2}+a_{\sigma}^{2} a_{\mu}^{2} c^{2}-a_{\sigma}^{4} a_{\mu}^{2} c^{2}-f^{2}\right\} \\
& \xi_{x}=F a \frac{a_{\sigma}^{2} s c}{2 f^{5}}\left\{-6 s^{6} a_{\sigma}^{8}+a_{\sigma}^{8} a_{\mu}^{2} s^{4}\left(4+3 c^{2}\right)-a_{\sigma}^{8} a_{\mu}^{4} c^{2} s^{2}+a_{\sigma}^{6} a_{\mu}^{4} c^{2} s^{2}\left(4-6 s^{2}\right)+a_{\sigma}^{6} a_{\mu}^{2} s^{4}\left(-3+15 s^{2}\right)\right. \\
& \left.+a_{\sigma}^{4} a_{\mu}^{4} 3 c^{2} s^{2}\left(4+3 s^{2}\right)-a_{\sigma}^{4} a_{\mu}^{6} 3 c^{4}\left(1+s^{2}\right)+a_{\sigma}^{2} a_{\mu}^{6} c^{4}\left(9-3 s^{2}\right)-3 a_{\mu}^{8} c^{6}\right\}, \\
& \xi_{y}=F a \frac{a_{\sigma}^{2} s c}{2 f^{3}}\left\{-a_{\sigma}^{4} a_{\mu}^{2} c^{2}+a_{\sigma}^{4} s^{2}\left(2+c^{2}\right)-a_{\mu}^{4} c^{4}+a_{\sigma}^{2} a_{\mu}^{2} c^{2}\left(3-2 s^{2}\right)\right\} \\
& \Omega_{1}=-F a^{2} \frac{3}{6} \frac{1}{f^{2}} \frac{s}{a_{\mu}^{2}}\left\{f^{2}-f a_{\sigma}^{4} c^{2}-\left(a_{\mu}^{2}-a_{\sigma}^{2}\right) c^{2}\left(f+a_{\sigma}^{4} s^{2}\right)\right\} \\
& \Omega_{2}=F a^{2} \frac{1}{6} \frac{1}{f^{4}}\left\{-3 s f^{2}\left(f+a_{\sigma}^{4} s^{2}\right)+a_{\sigma}^{2} c^{2}\left(3 a_{\sigma}^{2} s f+a_{\sigma}^{6} s^{3}\right) f+2\left(a_{\mu}^{2}-a_{\sigma}^{2}\right) c^{2}\left(3 f^{2} s+6 a_{\sigma}^{4} s^{3}+a_{\sigma}^{8} s^{5}\right)\right\} \\
& D_{x x}=F \frac{a^{3}}{24} \frac{1}{f^{5}}\left\{-4\left(3 a_{\sigma}^{2} s^{2} f+a_{\sigma}^{6} s^{4}\right) f^{2}+a_{\sigma}^{2} c^{2}\left(3 f^{2}+6 a_{\sigma}^{4} s^{2} f+a_{\sigma}^{8} s^{4}\right) f\right. \\
& \left.+2\left(a_{\mu}^{2}-a_{\sigma}^{2}\right) c^{2}\left(15 a_{\sigma}^{2} s^{2} f^{2}+10 a_{\sigma}^{6} s^{4} f+a_{\sigma}^{10} s^{6}\right)\right\} \\
& D_{y y}=F \frac{a^{3}}{24} \frac{1}{f^{5}} \frac{3 a_{\sigma}^{2}}{a_{\mu}^{2}}\left\{f^{4} c^{2}\right\} \\
& D_{x y}=F \frac{6 a^{3}}{24} \frac{1}{f^{5}} \frac{f^{2}}{a_{\mu}^{2}}\left\{-2\left(a_{\sigma}^{2} s^{2}\right) f^{2}+a_{\sigma}^{2} c^{2}\left(f^{2}+a_{\sigma}^{4} s^{2} f\right)+2\left(a_{\mu}^{2}-a_{\sigma}^{2}\right) c^{2}\left(3 a_{\sigma}^{2} s^{2} f+a_{\sigma}^{6} s^{4}\right)\right\} .
\end{aligned}
$$

In the above expressions, we have defined

$$
F \equiv \frac{J \epsilon p a}{\sigma \mu \sqrt{2 \pi f}} e^{-a_{\sigma}^{2} a_{\mu}^{2} c^{2} / 2 f} .
$$

and, as introduced in Appendix B,

$$
\begin{array}{r}
a_{\sigma} \equiv \frac{a}{\sigma}, a_{\mu} \equiv \frac{a}{\mu}, \\
s \equiv \sin \theta, c \equiv \cos \theta, \\
f \equiv a_{\sigma}^{2} s^{2}+a_{\mu}^{2} c^{2} .
\end{array}
$$

Equation (40) with the coefficients (41)-(55), fully describe the nonlinear time evolution of sputter eroded surfaces, provided that the leading relaxation mechanisms are thermally activated surface diffusion and ion-induced effective smoothing. Due to its highly nonlinear character, Eq. (40) can predict rather complex morphologies and dynamical behaviors. In the remainder of the paper we will focus on the physical interpretation of the coefficients (41)-(55), uncovering their dependence on the experimental parameters, and we discuss the morphologies described by Eq. (40). Consistent with the symmetries imposed by the geometry of the problem, the coefficients in Eq. 40 are symmetric under the transformation $y \rightarrow-y$ but not under $x \rightarrow-x$, while for $\theta \rightarrow 0$ the system is isotropic in the $x$ and $y$ directions, specifically $\gamma=\xi_{x}=\xi_{y}=\Omega_{1}=\Omega_{2}=0, \lambda_{x}=\lambda_{y}, \nu_{x}=\nu_{y}$, and $D_{x x}=D_{y y}=\frac{1}{2} D_{x y}$. 


\section{ANALYSIS OF THE GROWTH EQUATIONS}

This section is devoted to the study of the morphological properties predicted by Eq. (40). This is not a simple task, due to large number of linear and nonlinear terms, each of which influence the surface morphology. The complexity of the problem is illustrated by some special cases of Eq. (40), for which the behavior is better understood. For example, when nonlinear terms and the noise are neglected $\left(\xi_{x}=\xi_{y}=\lambda_{x}=\lambda_{y}=0, \eta=0\right)$, Eq. (40) reduces to a linear generalization of $\mathrm{BH}$ theory, which predicts ripple formation. It is also known that the isotropic KS equation, obtained by taking $\nu_{x}=$ $\nu_{y}, D_{x x}=D_{y y}=D_{x y} / 2$, and $\lambda_{x}=\lambda_{y}$, asymptotically predicts kinetic roughening, with morphology and exponents similar to those seen experimentally in ion sputtering [28,29]. For positive $\nu_{x}$ and $\nu_{y}$, Eq. (40) reduces to the anisotropic KPZ equation, whose scaling behavior is controlled by the sign of the product $\lambda_{x} \cdot \lambda_{y}[53]$. Finally, recent integration by Rost and Krug [65] of the noiseless anisotropic KS equation (i.e., when $\eta=0$ ) showed that when $\lambda_{x} \cdot \lambda_{y}<0$, ripples unaccounted for by the linear theory appear, their direction being rotated with respect to the ion direction.

To predict the morphology of ion-sputtered surfaces, we need to gain a full understanding of the behavior predicted by (40) in the physically relevant two dimensional case, going beyond the special cases. Help is provided by the recent numerical integration of (40) by Park et al. 100 that indicates a clear separation in time of the linear and nonlinear behaviors. The results show that before a characteristic time $t_{c}$ has been reached, the morphology is fully described by the linear theory, as if nonlinear terms were not present. However, after $t_{c}$ the nonlinear terms completely determine the surface morphology. These results offer a natural layout for our discussion. In section VIA we will limit our discussion to the linear theory. However, even in this case we have to distinguish four different cases, depending on whether the surface diffusion in the system is thermally generated or of the effective type associated with the ion erosion process. Consequently, in Sections VIA 1 - VIA2, we discuss the high temperature case, when relaxation is by thermal surface diffusion, treating separately the symmetric $(\sigma=\mu)$, and asymmetric $(\sigma \neq \mu)$ cases. Next we turn our attention to low temperature ripple formation, when surface relaxation is dominated by erosion, and we again distinguish the symmetric and asymmetric cases (Sects. VIA3 and VI A 4). Finally, Sections VIB 1 - VIB 4 are devoted to the effect of the nonlinear terms, addressing such important features as ripple stabilization, rotated ripples and kinetic roughening.

\section{A. Linear theory}

\section{Ripple formation at high temperatures: Symmetric case}

In this section we discuss the process of ripple formation in the symmetric case $\sigma=\mu$, when the relaxation is by thermally activated surface diffusion. Thus we assume that the magnitude of the thermally activated surface diffusion coefficient, $K$, is much larger than $D_{x x}, D_{x y}, D_{y y}$, generated by the ion bombardment process. This is always the case for high temperatures since $K$ increases as $(1 / T) \exp \left(-\Delta E / k_{B} T\right)$ with $T$, while ion induced effective smoothing terms are independent of $T$. Dropping the nonlinear terms in Eq. (40), we obtain

$$
\begin{aligned}
\frac{\partial h}{\partial t}= & -v_{0}+\gamma \frac{\partial h}{\partial x}+\nu_{x} \frac{\partial^{2} h}{\partial x^{2}}+\nu_{y} \frac{\partial^{2} h}{\partial y^{2}} \\
& +\Omega_{1} \frac{\partial^{3} h}{\partial x^{3}}+\Omega_{2} \frac{\partial^{3} h}{\partial x \partial^{2} y}-K \nabla^{4} h+\eta(x, y, t),
\end{aligned}
$$

where the coefficients can be obtained from Eqs. (41) (54) by taking $\sigma=\mu$ :

$$
\begin{aligned}
v_{0} & =F c, \\
\gamma & =F s\left(a_{\sigma}^{2} c^{2}-1\right), \\
\nu_{x} & =\frac{F a}{2}\left\{2 s^{2}-c^{2}-a_{\sigma}^{2} s^{2} c^{2}\right\}, \\
\nu_{y} & =-\frac{F a}{2} c^{2}, \\
\Omega_{1} & =-\frac{F a^{2} s}{2 a_{\sigma}^{2}}\left(1-a_{\sigma}^{2} c^{2}\right), \\
\Omega_{2} & =\frac{F a^{2}}{6 a_{\sigma}^{2}} s\left\{a_{\sigma}^{2}\left(3 c^{2}-3 s^{2}\right)+a_{\sigma}^{4} c^{2} s^{2}-3\right\} .
\end{aligned}
$$

Since the surface morphology depends on the signs and absolute values of the coefficients in Eq. (56), in the following we discuss in detail their behavior as functions of the angle of incidence $\theta$ and the reduced penetration depth $a_{\sigma}$.

Erosion velocity, $v_{0}$ : The $v_{0}$ term describes the erosion velocity of a flat surface. This term does not affect the ripple characteristics, such as ripple wavelength and ripple amplitude, and can be eliminated from the surface evolution equation by the coordinate transformation $\tilde{h}=h+v_{0} t$. This corresponds to a transformation to the coordinate frame moving with the eroded surface. However, since $v_{0}$ is the largest contribution to the erosion rate and is the only one that contributes in the linear theory, it is worthwhile to investigate its dependence on $\theta$ and $a_{\sigma}$. Fig. 6 shows the $v_{0}$ dependence on the angle of incidence $\theta$ for three different values of the reduced penetration depth $a_{\sigma}$. From Eq. (57), $v_{0}$ is positive for all $\theta$ and $a_{\sigma}$. In experiments $v_{0}(\theta)$ corresponds to the secondary ion yield variation with the incidence angle $\theta$, i.e. $v_{0}(\theta)=J Y_{\text {flat }}(\theta) / n$, where $n$ is the density of target atoms. 


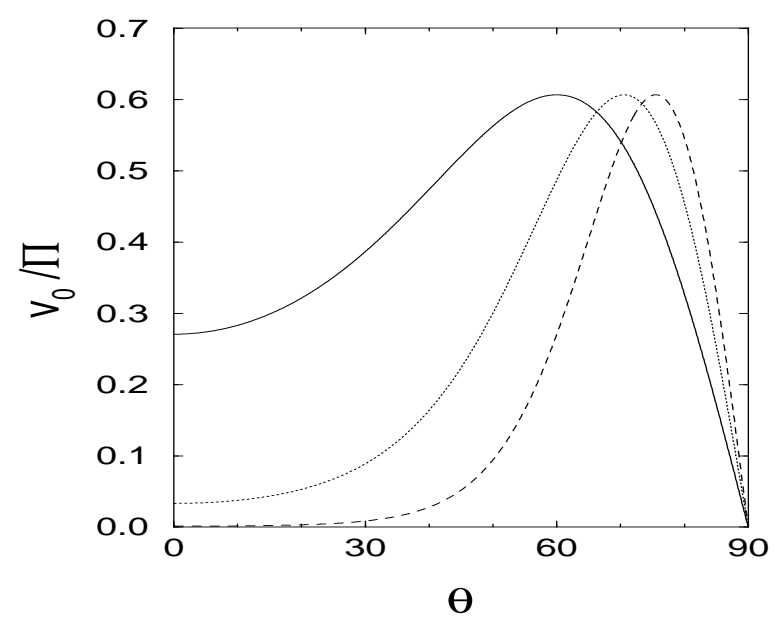

FIG. 6. The erosion velocity, $v_{0} / \Pi$ as a function of $\theta$. The three curves correspond to the reduced penetration depth $a_{\sigma}=1$ (solid line), $a_{\sigma}=2$ (dotted line), $a_{\sigma}=3$ (dashed line). The velocity has been normalized by a factor $\Pi=p \epsilon J /(\sqrt{2 \pi} a)$, independent of $\theta$.

Note that $v_{0}$ has the characteristic increasing part for small $\theta$, followed by saturation and decrease for large $\theta$, similar to the measured yield [2].

Traveling ripples, $\gamma, \Omega_{1}, \Omega_{2}$ : If we consider a periodic perturbation with wave vector $\left(q_{x}, q_{y}\right)$ in the form

$$
h=-v_{0} t+A \exp \left[i\left(q_{x} x+q_{y} y-\omega t\right)+r t\right],
$$

from Eq. (56) we obtain the mode velocity

$$
\omega=-\gamma q_{x}+\Omega_{1} q_{x}^{3}+\Omega_{2} q_{x} q_{y}^{2}
$$

and the growth rate

$$
r=-\left(\nu_{x} q_{x}^{2}+\nu_{y} q_{y}^{2}+K\left(q_{x}^{2}+q_{y}^{2}\right)^{2}\right)
$$

Thus the coefficients $\gamma, \Omega_{1}, \Omega_{2}$ contribute to the Fourier mode velocity $\omega$ in an anisotropic way that reflects the asymmetry of the $x$ and $y$ directions for oblique $(\theta \neq 0)$ ion incidence. The coefficients $\nu_{x}, \nu_{y}, K$, on the other hand, contribute to the growth rate of the mode amplitude. Carter [99,101 pointed out that dispersive terms, such as $\Omega_{1}$ and $\Omega_{2}$, destroy the translational invariance of the periodic morphology because the different modes travel with different velocities. Note, however, that the existence of a ripple structure means that there is essentially only one Fourier mode describing the surface morphology, which will thus move across the surface with velocity $\omega$. The coefficient $\gamma$ contributes only to the velocity of the ripples along the $x$ direction, leaving unaffected the $y$ component of the ripple velocity. Thus, as expected, $\gamma=0$ for normal incidence $(\theta=0)$. Similarly to the $v_{0}$ term, $\gamma$ does not affect the ripple characteristics and can actually be eliminated using the transformation $\tilde{h}=h(x-\gamma t, t)$.

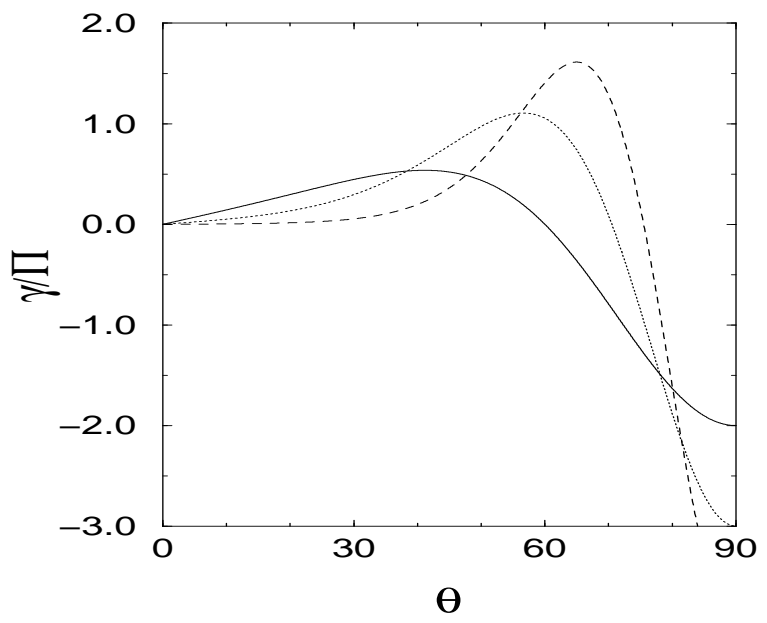

FIG. 7. The coefficient $\gamma / \Pi$ as a function of the angle of incidence $\theta$ for three reduced penetration depths: $a_{\sigma}=1$ (solid line); $a_{\sigma}=2$ (dotted line); $a_{\sigma}=3$ (dashed line).

As can be seen in Fig. 7, $\gamma$ can change sign with $\theta$, indicating that ripples travel in both positive and negative directions along the $x$ coordinate, depending on the angle of incidence and the penetration depth: ripples travel in the positive $x$ direction for small $\theta$ and in the negative $x$ direction for larger $\theta$. Travelling ripples were observed in numerical simulations of Koponen et al. 90.

As discussed above, the terms $\Omega_{1}, \Omega_{2}$ also contribute to the travelling of the ripples, and thus have no further effect on the surface morphology. Fig. 8 shows the coefficients $\Omega_{1}$ and $\Omega_{2}$ as functions of the angle of incidence $\theta$. We find that the absolute value of these coefficients at small angles is small compared to $\gamma$ (see Fig. 1), thus the main contribution to the ripple velocity comes from the $(\gamma \partial h / \partial x)$ term. On the other hand, for angles $\theta \geq 60^{\circ}$, these terms are comparable to or larger than $\gamma$.
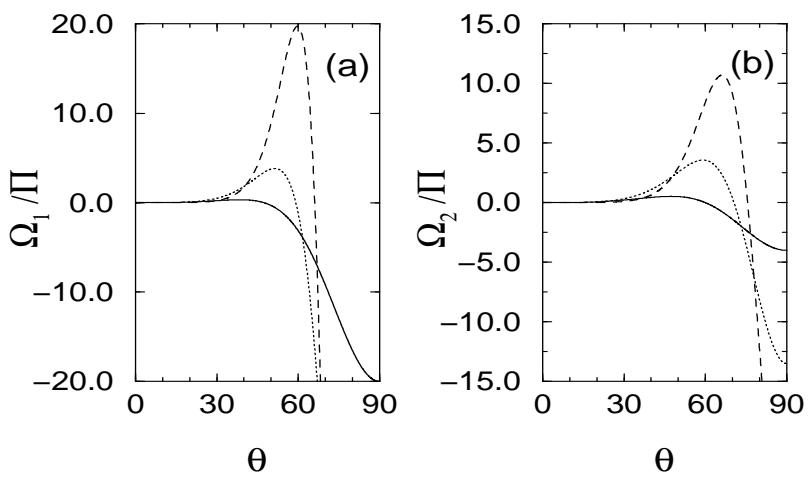

FIG. 8. The reduced third order coefficients $\Omega_{1} / \Pi$ (a) and $\Omega_{2} / \Pi$ (b) as functions of the angle of incidence $\theta$ for three reduced penetration depths: $a_{\sigma}=2$ (solid line); $a_{\sigma}=3$ (dotted line); $a_{\sigma}=4$ (dashed line). 

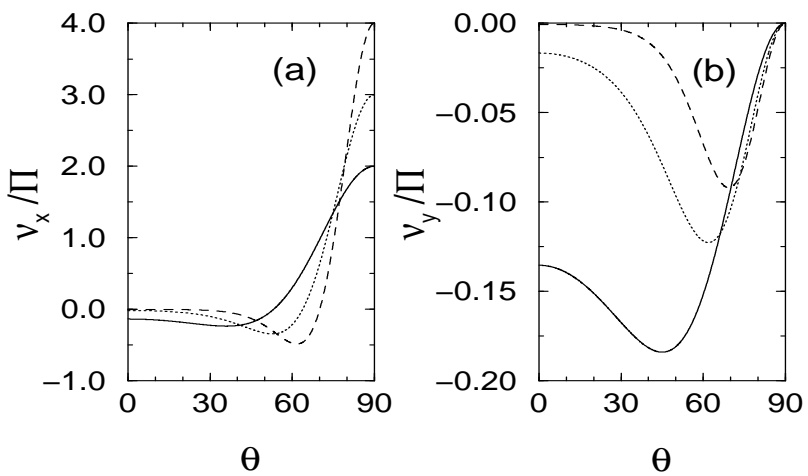

FIG. 9. The surface tension coefficients $\nu_{x} / \Pi$ (a) and $\nu_{y} / \Pi$ (b) as functions of the angle of incidence $\theta$ for three reduced penetration depths: $a_{\sigma}=2$ (solid line); $a_{\sigma}=3$ (dotted line); $a_{\sigma}=4$ (dashed line).

The coefficients $\nu_{x}$ and $\nu_{y}$ : As we discussed above (see Sect. IV B] the negative surface tension coefficients are the origin of the instability responsible for ripple formation. Consequently, they play a particularly important role in determining the surface morphology. The coefficients $\nu_{x}$ and $\nu_{y}$ are not equal due to the fact that the direction of the ion beam breaks the symmetry along the surface. As seen in Eq. (57), $\nu_{y}$ is always negative, while $\nu_{x}$ can change sign as $\theta$ and $a_{\sigma}$ vary, as shown in Fig. 9 . The sign and the magnitude of $\nu_{x}$ and $\nu_{y}$ determine both the wavelength and the orientation of the ripples.

Ripple wavelength and orientation: The experimental studies on ripple formation have mainly focused on the measurement of the ripple characteristics, such as the ripple wavelength and amplitude. Thus, a successful theory must address and predict these quantities. In the following we outline the method for calculating the ripple wavelengths $\ell_{x}$ and $\ell_{y}$. Taking into account the noisy character of Eq. (56), the experimentally observed ripple wavelength corresponds to the unstable Fourier mode which yields the maximum value of the structure factor. The structure factor, $S(\mathbf{q}, t)$, is calculated from the Fourier transform $h(\mathbf{q}, t)$ of the instantaneous surface profile and is defined as

$$
S(\mathbf{q}, t)=\langle h(-\mathbf{q}, t) h(\mathbf{q}, t)\rangle,
$$

where

$$
h(\mathbf{q}, t)=\int \frac{d \mathbf{r}}{(2 \pi)^{2}} \exp (i \mathbf{q r}) h(\mathbf{r}, t) .
$$

Fourier transforming Eq. (56) and inserting the expression for the Fourier transforms of $h(\mathbf{r}, t)$ into (61), we obtain

$$
S(\mathbf{q}, t)=\langle h(-\mathbf{q}, t) h(\mathbf{q}, t)\rangle=-\frac{J}{2} \frac{1-\exp (r(\mathbf{q}) t)}{r(\mathbf{q})},
$$

where $r$ is the growth rate of the mode $\mathbf{q}$ given by Eq. (60) and is positive for all unstable Fourier modes in the system. We find that, depending on the sign of $\nu_{x}$ and the relative magnitude of $\nu_{x}$ and $\nu_{y}$, we can distinguish two cases:

(i) For $\nu_{x}<\nu_{y}<0$, which, according to Eq. (57), holds when

$$
a_{\sigma}>\sqrt{\frac{2}{c^{2}}}
$$

the ripple structure is oriented in the $x$ direction, with ripple wavelength

$$
\ell_{x}=2 \pi \sqrt{\frac{2 K}{\left|\nu_{x}\right|}}
$$

This means that the maximum of $S(\mathbf{q}, t)$ is at $\left(\sqrt{\frac{\left|\nu_{x}\right|}{2 K}}, 0\right)$. To illustrate this, in Fig. 10 we show the dependence of the structure factor on the wavevectors $q_{x}$ and $q_{y}$. The contour plot indicates the existence of a global maximum at $\left(\sqrt{\frac{\left|\nu_{x}\right|}{2 K}}, 0\right)$, indicating that the ripples are oriented along the $x$ direction.

(ii) For $\nu_{x}>\nu_{y}$, which holds when

$$
a_{\sigma}<\sqrt{\frac{2}{c^{2}}}
$$

the ripple structure is oriented along the $y$-direction, with ripple wavelength

$$
\ell_{y}=2 \pi \sqrt{\frac{2 K}{\left|\nu_{y}\right|}} .
$$

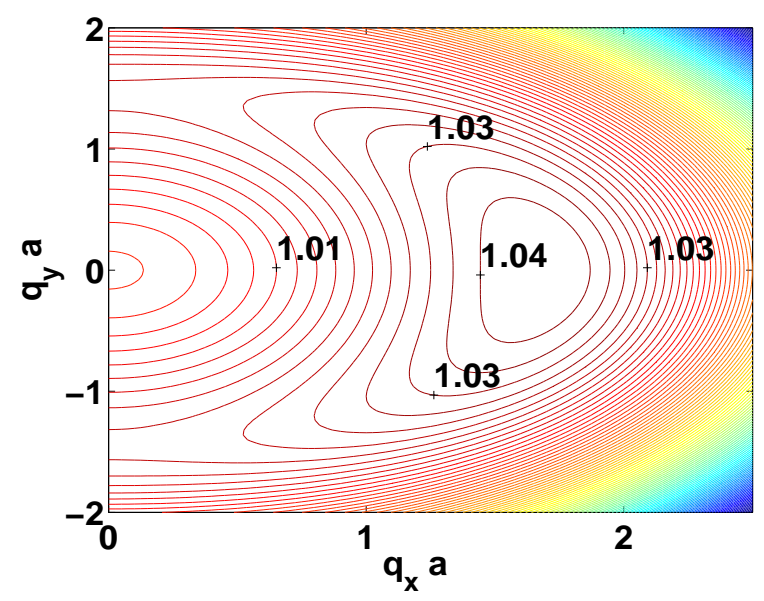

FIG. 10. Contour plot of the structure factor $2 S\left(q_{x}, q_{y}\right) / J$ as a function of the two dimensionless wavevectors $q_{x} a$ and $q_{y} a$ calculated for the angle of incidence $\theta=30^{\circ}$. The reduced coefficients $\nu_{x} / \Pi$ and $\nu_{y} / \Pi$ are obtained using Eq. (57), their values being $\nu_{x} / \Pi=-0.057$, and $\nu_{y} / \Pi=-0.0418$, while $K / \Pi$ is taken to be 0.01 . These parameter values correspond to Region I in Fig. 12 . 


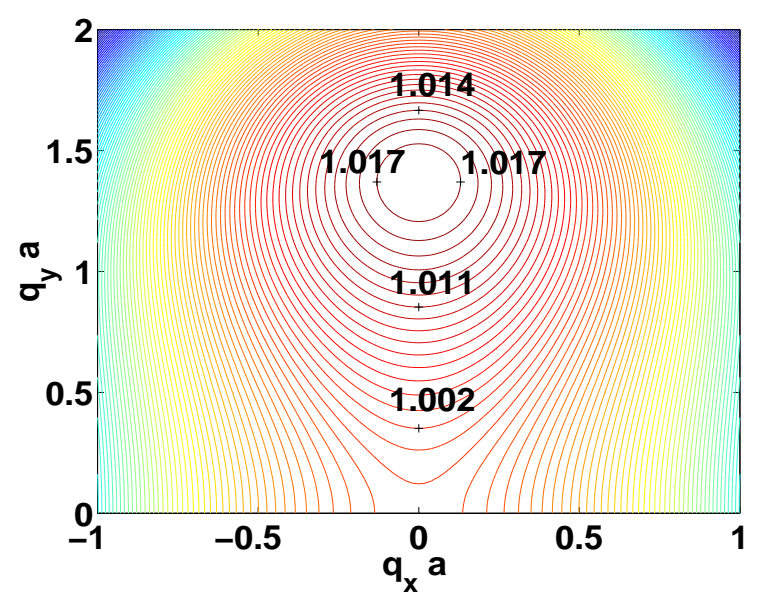

FIG. 11. Contour plot of the structure factor $2 S\left(q_{x}, q_{y}\right) / J$ as a function of the two dimensionless wavevectors $q_{x} a$ and $q_{y} a$ calculated for the angle of incidence $\theta=60^{\circ}$. The reduced coefficients $\nu_{x} / \Pi$ and $\nu_{y} / \Pi$ are obtained using Eq. (57), their values being $\nu_{x} / \Pi=0.0758$, and $\nu_{y} / \Pi=-0.0379$, while $K / \Pi$ is taken to be 0.01 . These parameter values correspond to Region II in Fig. 12.

Figure 11 shows an example of this regime, indicating the existence of a global maximum at point $\left(0, \sqrt{\frac{\left|\nu_{y}\right|}{2 K}}\right)$, corresponding to the ripple structure oriented along the $y$ direction.

Phase diagram for ripple orientation - The results obtained on ripple formation can be summarized in a $\left(\theta, a_{\sigma}\right)$ morphological phase diagram, shown in Fig. 12, which has the following regions:

Region I- For small $\theta$ both $\nu_{x}$ and $\nu_{y}$ are negative such that $\nu_{x}<\nu_{y}$, thus the ripples are oriented along the $x$ direction. Their wavelength is $\ell_{x}=2 \pi \sqrt{2 K /\left|\nu_{x}\right|}$.

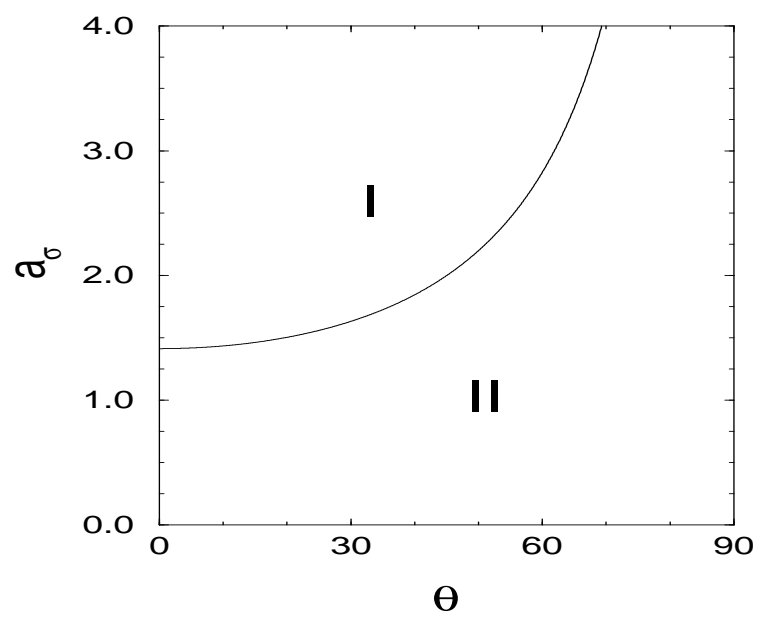

FIG. 12. Ripple orientation phase diagram for the isotropic case $\sigma=\mu=1$. Region I: $\nu_{x}<\nu_{y}<0$; Region II: $\nu_{x}>\nu_{y}$.

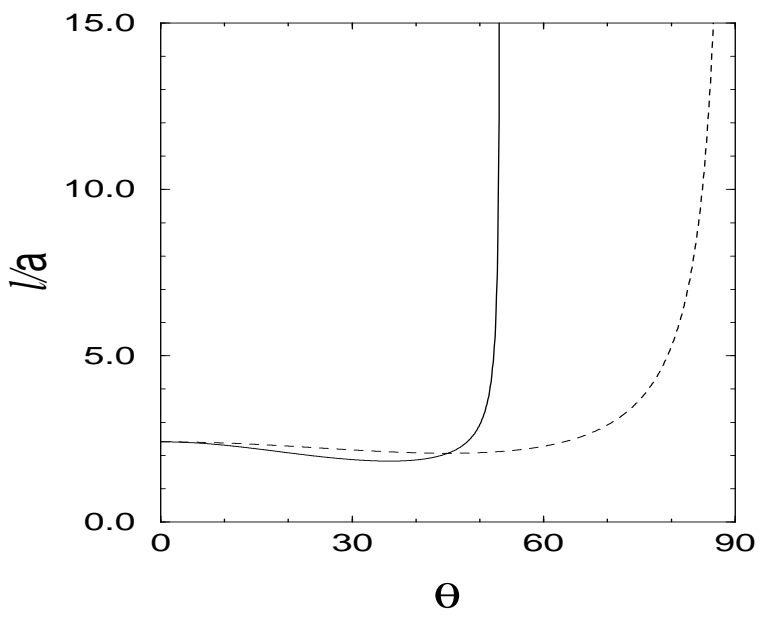

FIG. 13. Ripple wavelengths $\ell_{x}$ (solid line) and $\ell_{y}$ (dotted line) as functions of the angle of incidence $\theta$ for $K p \epsilon J /(\sqrt{2 \pi} a)=0.01$. The reduced penetration depth is taken as $a_{\sigma}=2$.

The amplitude of the ripples is expected to be weakly modulated by the larger wavelength $\ell_{y}=2 \pi \sqrt{2 K / \mid \nu_{y}} \mid$. The ripple amplitude grows as $h_{0} \sim \exp \left(r_{x} t\right)$, where $r_{x}=r\left(\sqrt{\frac{\left|\nu_{x}\right|}{2 K}}, 0\right)$ (see Eq. (58)). The boundary of this region is defined by $\nu_{x}\left(a_{\sigma}, \theta\right)=\nu_{y}\left(a_{\sigma}, \theta\right)$, i.e.

$$
a_{\sigma}=\sqrt{\frac{2}{c^{2}}}
$$

Region II-This region is characterized by $\nu_{y}<\nu_{x}$. The ripples are directed along the $y$ direction and have wavelength $\ell_{y}=2 \pi \sqrt{2 K / \mid \nu_{y}} \mid$. Note that Region II extends down to small values of the incidence angle $\theta$ for small enough reduced penetration depth $a_{\sigma}$. This somewhat unphysical result is a consequence of the assumption of a symmetric $(\sigma=\mu)$ distribution of deposited energy. We will see in the next section that the more physical asymmetric case with $\sigma>\mu$ leads in most cases to ripples only oriented along the $x$ direction for small enough angles of incidence, as generally observed.

Figure 13 shows the $\theta$ dependence of the ripple wavelengths along the $x$ and $y$ directions. In the framework of this model, where thermal surface diffusion is the only smoothing mechanism, the observed ripple orientation corresponds to the direction featuring the smallest value of $\ell$, and changes when $\ell_{x}=\ell_{y}$. The prediction for the ripple wavelength close to $90^{\circ}$ is questionable since reflection [69,70] and shadowing [102], not incorporated in the model, start to play an important role during ionbombardment at these high angles.

Summary: The dependence of $\ell$ on the main physical parameters characterizing the sputtering process is given by 


$$
\ell=2 \pi \sqrt{\frac{2 K}{|\nu|}} \sim \sqrt{\frac{2 K}{F a}}
$$

This prediction has a number of consequences, some of which have been verified experimentally (see Section VII):

(a) Since the penetration depth, $a$, is proportional to $\epsilon^{2 m}$ (see IVE), and $\sigma \sim \mu \sim a$, we have $a_{\sigma} \sim$ const, and $F \sim(\epsilon a) /(\sigma \mu) \sim \epsilon^{1-2 m}$, which is independent of $\epsilon$, when $m=1 / 2$. Consequently

$$
\ell \sim \epsilon^{-1 / 2},
$$

i.e. the ripple wavelength is expected to decrease with the ion energy.

(b) Taking into account that $K$ is independent of the flux and $\nu \sim J$, we obtain that the ripple wavelength is also a decreasing function of the incident ion flux, given by

$$
\ell \sim \frac{1}{J^{1 / 2}}
$$

(c) As was mentioned above, the negative surface tension is the origin of the instability leading to ripple formation. When both $\nu_{x}$ and $\nu_{y}$ are negative the experimentally observed ripple structure has the direction for which the growth rate $r$ is largest. However, in general, we expect a superposition of both wavelengths, where the long wavelength will appear as a modulation of the ripple amplitude. Indeed, such modulations have been observed both experimentally and numerically 100 .

(d) An important prediction of this model, illustrated in Fig. 13, is the existence of the critical angle $\theta_{c}$ where the ripple orientation changes. In the case when surface diffusion is thermally activated, this transition coincides with the condition $\nu_{x}=\nu_{y}$.

\section{Ripple formation at high temperatures: Asymmetric case}

The results of the previous section were derived for the isotropic case, $\sigma=\mu$. While this approximation considerably simplifies our discussion, most systems present some anisotropy in the deposited energy distribution. In this section we demonstrate that the existence of anisotropy does not modify the overall qualitative result on the existence of the two parameter regions corresponding to ripples oriented along the $x$ or $y$ directions. However, anisotropy does change the numerical value of the ripple wavelength and the exact boundary between the two morphological regions: we demonstrate that, for large enough anisotropies, if the incidence angle is small only ripples oriented along the $x$ direction are possible.

Fig. 14 shows the coefficients $\nu_{x}$ and $\nu_{y}$, given by Eq. (57), as functions of the angle of incidence $\theta$, for three different degrees of asymmetry $\tau=\sigma / \mu$ in the physical
38, 69, 70 $\tau>1$ range. As one can observe the qualitative behavior of $\nu_{x}$ and $\nu_{y}$ is similar to that observed in the symmetric case. One interesting feature, however, must be emphasized: the increasing asymmetry leads to larger ripple wavelength, since the absolute values of $\nu_{x}$ and $\nu_{y}$ decrease. With respect to the the third order linear terms $\Omega_{1}$ and $\Omega_{2}$, their behavior as functions of the angle of incidence can be also seen to be qualitatively analogous to the symmetric case. Thus the asymmetry does not change our conclusions regarding the travelling ripples. Ripple wavelength: The calculation of ripple characteristics in the asymmetric case is identical to the one used in the symmetric case. Therefore, we limit ourselves to the presentation of the results. Again, there are two possible ripple directions, and the dominant one can be found from the maximum of the structure factor $(63)$ or, as can be seen to be equivalent, from the maximum of the growth rate (60):

(i) When $\nu_{x}<\nu_{y}<0$, i.e.,

$$
a_{\sigma}>\sqrt{\frac{s^{2}\left(2+c^{2}\right)+\tau^{2} c^{2}\left(1+2 c^{2}\right)-\tau^{4} c^{4}}{\tau^{2} c^{2}}},
$$

the ripple structure is oriented along the $x$-direction with ripple wavelength $\ell_{x}=2 \pi \sqrt{\frac{2 K}{\left|\nu_{x}\right|}}$.

(ii) When $\nu_{x}>\nu_{y}$, i.e.,

$$
a_{\sigma}<\sqrt{\frac{s^{2}\left(2+c^{2}\right)+\tau^{2} c^{2}\left(1+2 c^{2}\right)-\tau^{4} c^{4}}{\tau^{2} c^{2}}}
$$

the ripples are oriented along the $y$-direction, with ripple wavelength $\ell_{y}=2 \pi \sqrt{\frac{2 K}{\left|\nu_{x}\right|}}$.
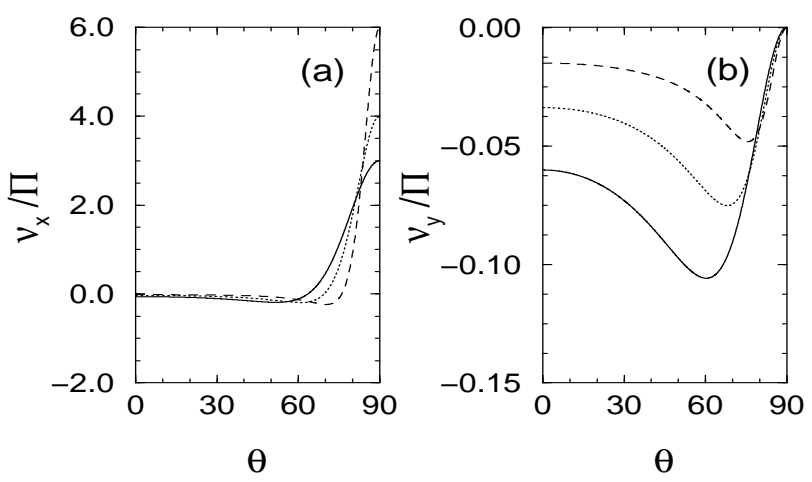

FIG. 14. The effective surface tensions $\nu_{x} / \Pi$ (a) and $\nu_{y} / \Pi$ (b) plotted as functions of the incidence angle $\theta$ in the asymmetric case. The curves correspond to values of the asymmetry parameter $\tau=1.5$ (solid line), $\tau=2$ (dotted line), $\tau=3$ (dashed line), with $a_{\sigma}=2$. 
Phase diagram for ripple orientation: To consider the effect of asymmetry on the different regimes in ion sputtering, we have studied the ripple orientation phase diagram for different values of $\tau$. As $\tau$ changes, we find a smooth evolution which does not uncover any new morphological regime. However, the topology of the phase diagram does change as $\tau$ increases. For $\tau<\sqrt{3}$ the topology of the phase diagram is similar to the symmetric case (see Fig. 12). As Fig. 15 illustrates, for $\tau \geq \sqrt{3}$ the ripples oriented along the $y$ direction, predicted by the linear theory for small enough $\theta$ and $a_{\sigma}$, are absent, which is consistent with most experimental observations. The characteristics of Region $I$ and Region $I I$ of the phase diagram are the same as in the symmetric case.

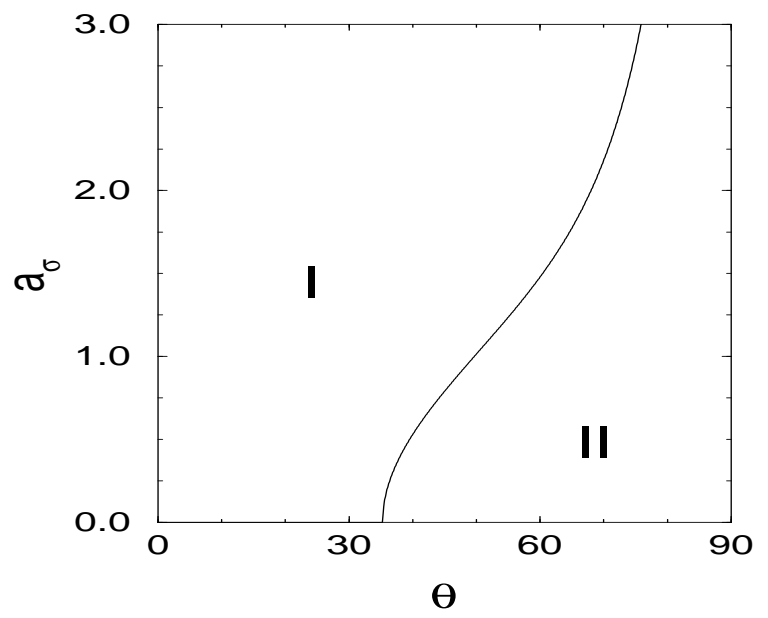

FIG. 15. Ripple orientation phase diagram for the asymmetric case with asymmetry parameter $\tau=2$ and $a_{\sigma}=2$. Region I: $\nu_{x}<\nu_{y}<0$; Region II: $\nu_{x}>\nu_{y}$.

\section{Ripple formation at low temperatures: Symmetric case}

In the previous two sections we discussed the process of ripple formation when the origin of surface smoothing is surface diffusion, described by the $-K \nabla^{4} h$ term. However, in the series expansion of the erosion velocity we found linear fourth order terms of the form $-D_{x x} \partial_{x}^{4} h$, $-D_{x y} \partial_{x}^{2} \partial_{y}^{2} h$, and $-D_{y y} \partial_{y}^{4} h$, which are formally equivalent to the thermally induced surface diffusion terms. These terms arise as a higher order correction to the local surface curvature, being fully determined by the process of surface erosion. Consequently, these terms do not imply actual mass transport along the surface, as thermal surface diffusion does. In this section we show that, in some parameter regions, these terms have a smoothing effect that counteracts the erosion instability, in such a way that they can also lead to ripple formation. We believe this explains the ripples observed at low temperatures both experimentally 12 and in computer simulations 90 .

Neglecting the thermally induced relaxation terms (i.e, taking $K=0$ ), nonlinear terms and the terms $v_{0}, \gamma, \Omega_{1}$ and $\Omega_{2}$, that do not affect the ripple characteristics, from Eq. (40) we obtain the linear equation

$$
\begin{aligned}
\frac{\partial h}{\partial t}= & \nu_{x} \frac{\partial^{2} h}{\partial x^{2}}+\nu_{y} \frac{\partial^{2} h}{\partial y^{2}}-D_{x x} \frac{\partial^{4} h}{\partial x^{4}} \\
& -D_{x y} \frac{\partial^{4} h}{\partial x^{2} \partial y^{2}}-D_{y y} \frac{\partial^{4} h}{\partial y^{4}}+\eta(x, y, t)
\end{aligned}
$$

The expressions for the coefficients of the ion-induced effective smoothing terms can be obtained from Eqs. (51)(53) using $\sigma=\mu$ :

$$
\begin{aligned}
D_{x x} & =\frac{F a^{3}}{24 a_{\sigma}^{2}}\left\{a_{\sigma}^{4} s^{4} c^{2}+a_{\sigma}^{2}\left(6 c^{2} s^{2}-4 s^{4}\right)+3 c^{2}-12 s^{2}\right\} \\
D_{x y} & =\frac{F a^{3}}{24 a_{\sigma}^{2}} 6\left\{a_{\sigma}^{2} s^{2} c^{2}+c^{2}-2 s^{2}\right\} \\
D_{y y} & =\frac{F a^{3}}{24 a_{\sigma}^{2}} 3 c^{2} .
\end{aligned}
$$

From Eq. 75, $D_{y y}$ is always positive, while $D_{x y}$ and $D_{x x}$ change sign with $\theta$. As we discuss below, the positive $D_{x x}$ and $D_{y y}$ coefficients play a role similar to thermally activated surface diffusion. Furthermore, the absolute value of $D_{x x}$ is comparable with the thermally activated surface diffusion coefficient even at high temperatures (see Sect. VII).

Ripple wavelength and orientation: The ripple wavelength and orientation can be calculated following the arguments presented in Section VI A 1, being determined by the maxima of the structure factor $S(\mathbf{q}, t)$. The growth rate $r$ is now given by

$$
\begin{aligned}
r\left(q_{x}, q_{y}\right)= & -\left(\nu_{x} q_{x}^{2}+\nu_{y} q_{y}^{2}+D_{x x} q_{x}^{4}\right. \\
& \left.+D_{x y} q_{x}^{2} q_{y}^{2}+D_{y y} q_{y}^{4}\right)
\end{aligned}
$$

In principle the asymmetry of the $D_{i j}$ coefficients may lead to maxima of $S(\mathbf{q}, t)$ at nonzero $q_{x}$ and $q_{y}$ values, which correspond to ripples forming a nonzero angle with both the $x$ and $y$ directions. However, straightforward calculations indicate that the following condition holds

$$
D_{x y} \nu_{y}=2 \nu_{x} D_{y y}
$$

for all values of $a_{\sigma}$ and $\theta$ in (57) and (75). This identity implies that no extrema $\left(q_{x}^{*}, q_{y}^{*}\right)$ of $S(\mathbf{q}, t)$ exist other than of the form $\left(q_{x}^{*}, 0\right)$ or $\left(0, q_{y}^{*}\right)$. Of these two solutions the one with the largest positive value of $r\left(q_{x}, q_{y}\right)$ corresponds to the observed ripple structure. For small angles of incidence so that $D_{x x} \geq 0$ (Region I in Fig. 16), 


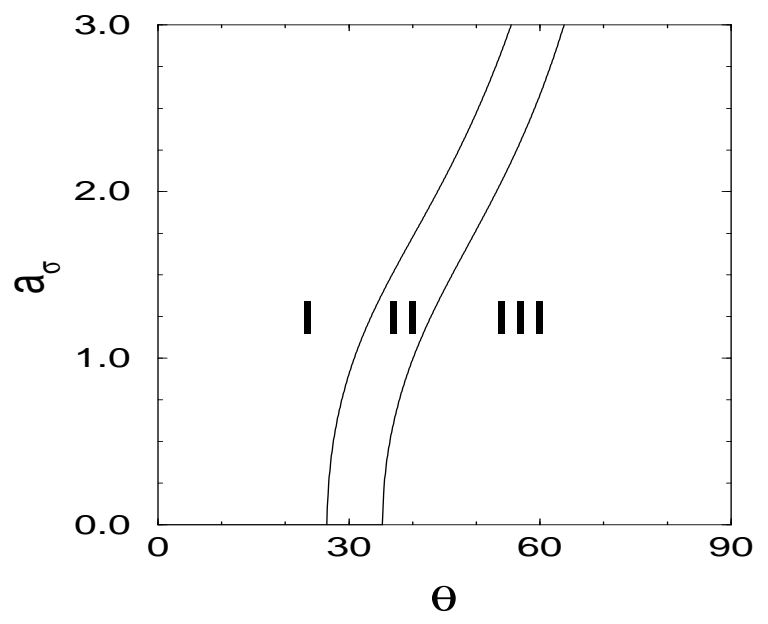

FIG. 16. Morphological phase diagram in the symmetric case $\sigma=\mu=1$. Different regions correspond to: Region I: $\nu_{x}<0, \nu_{y}<0, D_{x x}>0, D_{y y}>0$ and $r_{x}>r_{y}$; Region II: $\nu_{x}<0, \nu_{y}<0, D_{x x}<0, D_{y y}>0$; Region III: $\nu_{x}>0, \nu_{y}<0$, $D_{x x}<0$ and $D_{y y}>0$.

it can be easily seen that $\nu_{x}<0$, and the absolute maximum of $S(\mathbf{q}, t)$ is at $\left(q_{x}^{*}, 0\right)$ with $q_{x}^{*}=\sqrt{\left|\nu_{x}\right| / 2 D_{x x}}$, thus the ripple structure is aligned along the $x$ direction (see for example Fig. 17).

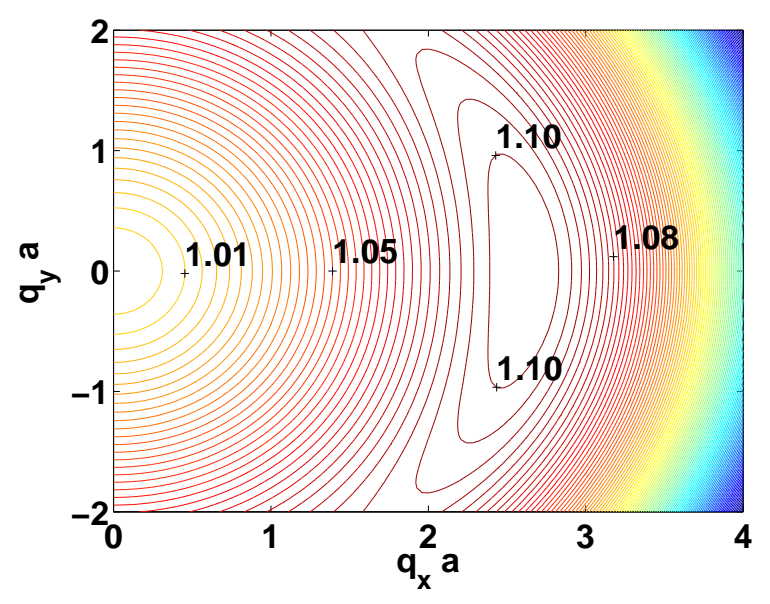

FIG. 17. Contour plot of the structure factor $2 S\left(q_{x}, q_{y}\right) / J$ shown as a function of the two dimensionless wavevectors $q_{x} a$ and $q_{y} a$ calculated for the angle of incidence $\theta=30^{\circ}$. The reduced coefficients $\nu_{x} / \Pi$ and $\nu_{y} / \Pi$ are obtained using Eq. (57), their values being $\nu_{x} / \Pi=-0.0578$ and $\nu_{y} / \Pi=-0.0418$. These parameter values correspond to Region I in Fig. 16.

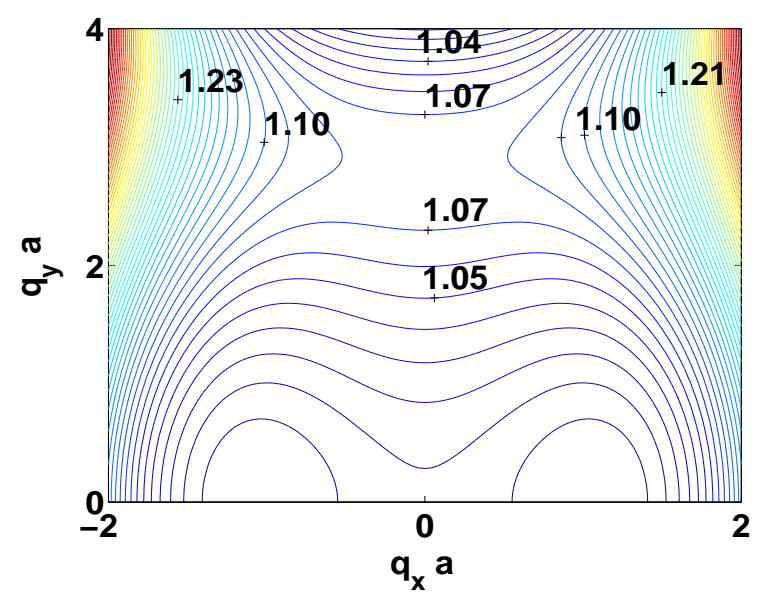

FIG. 18. Contour plot of the structure factor $2 S\left(q_{x}, q_{y}\right) / J$ shown as a function of the two dimensionless wavevectors $q_{x} a$ and $q_{y} a$ calculated for the angle of incidence $\theta=30^{\circ}$. The reduced coefficients $\nu_{x} / \Pi$ and $\nu_{y} / \Pi$ are obtained using Eq. (57), their values being $\nu_{x} / \Pi=-0.0758$ and $\nu_{y} / \Pi=-0.0379$. These parameters values correspond to Region II in Fig. 16.

Crossing the $D_{x x}=0$ line into Region II in Fig. 16 the $\left(q_{x}^{*}, 0\right)$ solution disappears, the structure factor having an extremum at $\left(0, q_{y}^{*}\right)$, with $q_{y}^{*}=\sqrt{\left|\nu_{y}\right| / 2 D_{y y}}$. However, this extremum is not a global maximum, see for example Fig. 18. The lower boundary of Region II is provided by the condition $\nu_{x}=0$. When we cross it (entering Region III in Fig. 16), we have $D_{x x}<0$ and $\nu_{x}>0$. Under this condition, again, there exists an extremum of $S(\mathbf{q}, t)$ at $\left(q_{x}^{*}, 0\right)$, with $q_{x}^{*}=\sqrt{\nu_{x} / 2\left|D_{x x}\right|}$. However, the structure factor takes its absolute maximum at $\left(0, q_{y}^{*}\right)$.

Phase diagram for ripple orientation: In summary, three different regions can be determined in the morphological phase diagram shown in Fig. 16 for the case of ion-induced effective smoothing in the symmetric $\sigma=\mu$ case.

Region $I$ - In this region, the surface tension coefficients $\nu_{x}$ and $\nu_{y}$ are negative, while $D_{x x}$ and $D_{y y}$ are both positive. The observed ripple structure corresponds to the maximum of $S(\mathbf{q}, t)$, which indicates that the ripple structure is oriented along the $x$ direction. The lower boundary of this region, separating it from Region II, is given by the $D_{x x}\left(a_{\sigma}, \theta\right)=0$ line or, equivalently, by

$$
a_{\sigma}=\sqrt{\frac{\left(2 s^{2}-3 c^{2}\right)+\sqrt{6 c^{4}+4 s^{4}}}{s^{2} c^{2}}} .
$$

Region $I I-$ In this region both $D_{x x}$ and $\nu_{x}$ are negative. This region is bounded below by the $\nu_{x}\left(a_{\sigma}, \theta\right)=0$ line, given by

$$
a_{\sigma}=\sqrt{\frac{\left(2 s^{2}-c^{2}\right)}{s^{2} c^{2}}} .
$$

In a continuum description, the maximum of $r(\mathbf{q})$ is at infinite $\mathbf{q}$, thus our theory possibly breaks down in the 
sense that not even non-linear effects can be expected to stabilize the surface under such conditions. In such a case, a higher order Taylor expansion should be carried out in Sec. $\mathrm{V}$ in order to be able to describe our system. Additional effects, such as shadowing, could also start to play a role under such conditions.

Region $I I I$ - In this region $D_{x x}$ is negative and $\nu_{x}$ is positive. Thus the instability given by the negative $D_{x x}$ is smoothed out by the positive $\nu_{x}$. Since the structure factor takes on its absolute maximum at the finite wave vector $\left(0, q_{y}^{*}\right)$, in principle there is still a ripple structure oriented along the $y$ direction. However, remarks similar to those made in Region II might apply here, since we still have $D_{x x}<0$.

Summary: In the presence of ion-induced effective smoothing the dependence of the ripple wavelength on the physical parameters is different from the case of thermal surface diffusion (see Sect. VI A 1). Here we summarize some of the differences.

(a) The dependence of $\ell$ on the ion energy is given by

$$
\ell=\sqrt{\frac{2 D}{|\nu|}} \sim \sqrt{\frac{F a^{3}}{F a}} \sim a \sim \epsilon^{2 m},
$$

indicating that the ripple wavelength depends linearly on the penetration depth $a$. This is very different from the behavior predicted by Eq. (56), derived for thermal surface diffusion, and indicates that monitoring the ripple wavelength dependence on $\epsilon$ can be used to identify the dominant smoothing mechanism. Such a linear behavior of $\ell$ on $\epsilon$ has indeed been seen experimentally (see Section III A).

(b) From Eq. (80) it also follows that the ripple wavelength is independent of the incident ion flux. This prediction is again quite different from the case dominated by thermal surface diffusion, given by Eq. (71). Such a flux independent behavior has been observed experimentally (see Section III A).

Finally, analogues of characteristics (c) and (d), discussed in Sect. VI A 1, apply here as well.

\section{Ripple formation at low temperatures: Asymmetric case}

In this section we discuss the effect of asymmetry $(\sigma \neq \mu)$ of the energy distribution on the morphological regimes predicted by Eq. (74). We find that the coefficients of Eq. (74) vary slowly with the asymmetry, but this does not change the qualitative picture presented in the previous section, regarding the ripple wavelength and orientation, or the major morphological regimes found in the isotropic case, including the conditions of validity of our continuum theory. Specifically, we find that the asymmetry enlarges the region in $\theta$ where $D_{x x}$ and $D_{y y}$ are positive, thus shifting Region $I I$ to larger values of $\theta$.
Phase diagram for ripple orientation - The topology of the morphological phase diagram and the characteristics of the three main regions are not changed by the asymmetry. We find that the only effect of the asymmetry is to move the boundaries smoothly to larger values of $\theta$ as $\tau$ increases. The condition $D\left(a_{\sigma}, \theta\right)=0$ (see Eq. (78)) now takes the form

$$
a_{\sigma}=\sqrt{\left(s^{2}+\tau^{2} c^{2}\right) \frac{\left(2 s^{2}-3 \tau^{2} c^{2}\right)+\sqrt{6 \tau^{4} c^{4}+4 s^{4}}}{\tau^{2} s^{2} c^{2}}},
$$

and the condition $\nu_{x}\left(a_{\sigma}, \theta\right)=0$ (see Eq. (79) ) becomes

$$
a_{\sigma}=\sqrt{\frac{2 s^{4}+\tau^{2} c^{2} s^{2}-\tau^{4} c^{4}}{\tau^{2} s^{2} c^{2}}} .
$$

\section{B. Nonlinear theory}

As we demonstrated in the previous section, linear theory can predict many features of ripple formation, such as the ripple wavelength and orientation, both at high and low temperatures. However, a number of important experimental features are incorrectly predicted by linear theory. They include the stabilization of the ripple amplitude (according to the linear theory the amplitude increases indefinitely at an exponential rate) or the presence of kinetic roughening (completely unaccounted for by the linear approach). To account for these features, we have to consider the effect of the nonlinear terms. Consequently, this section is devoted to the effect of the nonlinear terms on the surface morphology. There is an important difference between the linear and nonlinear theories: while all predictions of the linear theory can be calculated analytically (as we demonstrated in the previous section), the discussion of the nonlinear effects requires a combination of analytical and numerical tools. Even with these tools, the understanding of the nonlinear effects is far less complete than that of the linear theory.

\section{High temperature morphology: Symmetric case}

In the high temperature regime, where thermal surface diffusion dominates over ion-induced effective smoothing, the nonlinear equation of the interface evolution has the form

$$
\begin{aligned}
\frac{\partial h}{\partial t}= & -v_{0}+\gamma \frac{\partial h}{\partial x}+\xi_{x}\left(\frac{\partial h}{\partial x}\right)\left(\frac{\partial^{2} h}{\partial x^{2}}\right) \\
& +\xi_{y}\left(\frac{\partial h}{\partial x}\right)\left(\frac{\partial^{2} h}{\partial y^{2}}\right)+\nu_{x} \frac{\partial^{2} h}{\partial x^{2}} \\
& +\nu_{y} \frac{\partial^{2} h}{\partial y^{2}}+\frac{\lambda_{x}}{2}\left(\frac{\partial h}{\partial x}\right)^{2}+\frac{\lambda_{y}}{2}\left(\frac{\partial h}{\partial y}\right)^{2} \\
& +\Omega_{1} \frac{\partial^{3} h}{\partial x^{3}}+\Omega_{2} \frac{\partial^{3} h}{\partial x \partial y^{2}}-K \nabla^{4} h+\eta(x, y, t),
\end{aligned}
$$


where the coefficients of the linear terms, $v_{0}, \gamma, \nu_{x}, \nu_{\nu}$ $\Omega_{1}, \Omega_{2}$, and $K$ have been discussed in Sections VIA 1 and VIA2. The coefficients of the nonlinear terms in the symmetric case $(\sigma=\mu)$ are

$$
\begin{aligned}
\lambda_{x} & =\frac{F}{2} c\left\{a_{\sigma}^{2}\left(3 s^{2}-c^{2}\right)-a_{\sigma}^{4} s^{2} c^{2}\right\}, \\
\lambda_{y} & =-\frac{F}{2} c\left\{a_{\sigma}^{2} c^{2}\right\}, \\
\xi_{x} & =\frac{F a}{2} s c\left\{-a_{\sigma}^{4} c^{2} s^{2}+a_{\sigma}^{2}\left(4 s^{2}-3 c^{2}\right)+6\right\}, \\
\xi_{y} & =\frac{F a}{2} s c\left\{2-a_{\sigma}^{2} c^{2}\right\} .
\end{aligned}
$$

Next we discuss the physical interpretation and the behavior of these coefficients as functions of $\theta$ and $a_{\sigma}$.

The coefficients $\xi_{x}$ and $\xi_{y}$ : Fig. 19 shows the nonlinear coefficients $\xi_{x}$ and $\xi_{y}$ as functions of the angle of incidence $\theta$. As the numerical analysis of Eq. (83) shows, these nonlinearities are responsible for the development of overhangs on the surface 103. Even though the $\xi_{x}$ and $\xi_{y}$ terms are expected not to determine the asymptotic scaling behavior, they can play a relevant role at intermediate time scales after the development of the ripple structure, particularly in the region of large $\theta$. The precise contribution of these nonlinearities to the surface dynamics is currently under investigation 103].

The coefficients $\lambda_{x}$ and $\lambda_{y}$ : As we discussed in Sec. IV A 2, the morphology of the surface described by Eq. (83) depends on the relative signs of the the nonlinear terms $\lambda_{x}$ and $\lambda_{y}$. As it is evident from Eq. (44), $\lambda_{y}$ is negative for all angles of incidence and penetration depths.
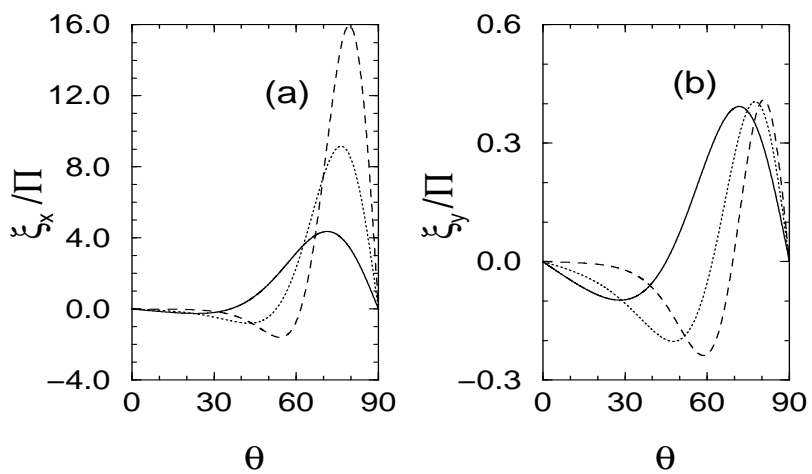

FIG. 19. The reduced coefficients $\xi_{x} / \Pi$ (a) and $\xi_{y} / \Pi$ (b) shown as functions of $\theta$ for different values of the reduced penetration depths: $a_{\sigma}=2$ (solid line); $a_{\sigma}=3$ (dotted line); $a_{\sigma}=4$ (dashed line).
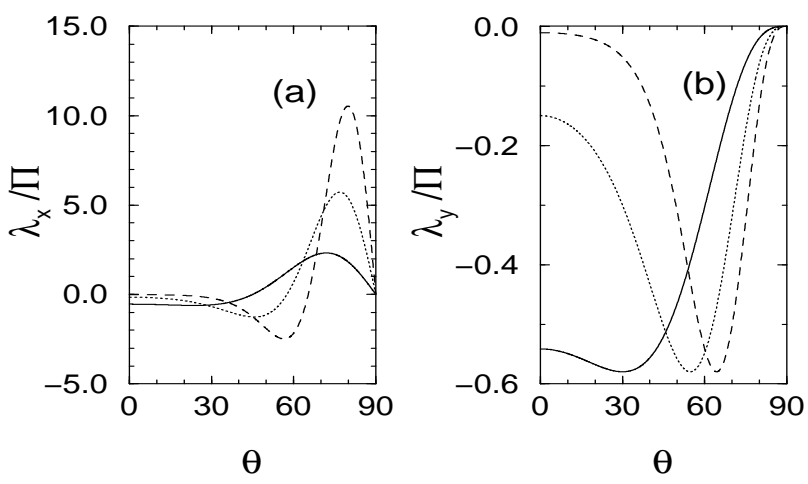

FIG. 20. The reduced coefficients $\lambda_{x} / \Pi$ (a) and $\lambda_{y} / \Pi$ (b) shown as functions of $\theta$. The different curves correspond to different values of the reduced penetration depth: $a_{\sigma}=2$ (solid line); $a_{\sigma}=3$ (dotted line); $a_{\sigma}=4$ (dashed line).

However, as shown in Fig. 20, the sign of $\lambda_{x}$ depends on $\theta$ and $a_{\sigma}: \lambda_{x}$ is negative for small $\theta$ and changes sign for larger angles of incidence. In principle the nonlinear terms completely determine the surface morphology. Since the nonlinear terms are always present, an important question is whether the linear regimes are relevant at all. Recent results by Park et al. [100] indicate that, while the nonlinear effects indeed change the surface morphology, the regime described by the linear terms is still visible for a wide range of parameters. By numerically integrating Eq. (83) they have shown that there is a clear separation of the linear and nonlinear regimes in time: for times up to a crossover time $t_{c}$ the surface erodes as if the nonlinear terms would be completely absent, following the predictions of the linear theory. After $t_{c}$, however, the nonlinear terms take over and completely determine the surface morphology. The transition from the linear to the nonlinear regime can be seen either by monitoring the surface width (which is proportional to the ripple amplitude) or the erosion velocity. The simulations indicate that the width increases exponentially with time, as predicted by the linear theory, until $t_{c}$, after which the width growths at a much slower rate [100]. This transition is typically accompanied by the disappearance of ripples predicted by the linear theory and the appearance of either kinetic roughening or of a new rotated ripple structure. The erosion velocity is constant in the linear regime (before $t_{c}$ ), while it increases or decreases after $t_{c}$, depending on the relative signs of the nonlinear terms.

Crossover time: The crossover time $t_{c}$ from the linear to the nonlinear behavior can be estimated 100 by comparing the strength of the linear terms with that of the nonlinear terms. Let the typical surface width at the crossover time $t_{c}$ be $W_{o}=\sqrt{W^{2}\left(L, t_{c}\right)}$. Then from the linear equation we obtain 


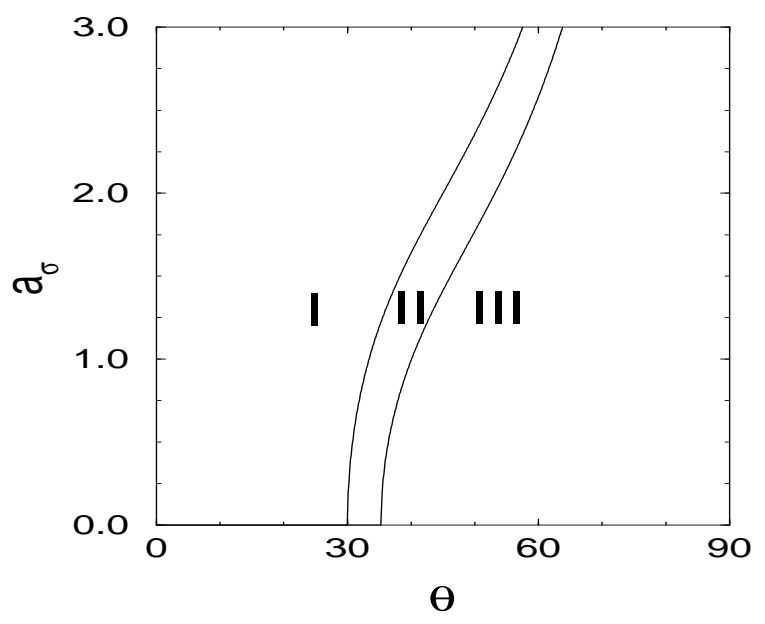

FIG. 21. Phase diagram for the isotropic case $\sigma=\mu=1$. Region I: $\nu_{x}<0, \nu_{y}<0, \lambda_{x}<0, \lambda_{y}<0$; Region II: $\nu_{x}<0$, $\nu_{y}<0, \lambda_{x}>0, \lambda_{y}<0$; Region III: $\nu_{x}>0, \nu_{y}<0, \lambda_{x}>0$, $\lambda_{y}<0$.

$$
W_{o} \sim \exp \left(\nu t_{c} / \ell^{2}\right)
$$

while from $\partial_{t} h \sim \lambda(\nabla h)^{2}$ we estimate

$$
W_{o} / t_{c} \sim \lambda W_{o}^{2} / \ell^{2}
$$

Combining these relations we obtain

$$
t_{c} \sim\left(K / \nu^{2}\right) \ln (\nu / \lambda)
$$

In this expression, $\nu, K$, and $\lambda$ correspond to the direction parallel to the ripple orientation. The predicted $\lambda$ dependence of $t_{c}$ has been confirmed by numerical simulations 100.

Surface morphology: The surface morphology in the nonlinear regime depends on the relative signs of $\nu_{x}, \nu_{y}$, $\lambda_{x}$ and $\lambda_{y}$. The different morphological regimes can be summarized in a phase diagram, shown in Fig. 21. Next we discuss each of the phases separately.

Region $I$ - For small $\theta$ the nonlinear terms $\lambda_{x}$ and $\lambda_{y}$ have the same (negative) sign, the boundary of this region being given by the condition that $\lambda_{x}\left(a_{\sigma}, \theta\right)=0$, or equivalently

$$
a_{\sigma}=\sqrt{\frac{3 s^{2}-c^{2}}{c^{2} s^{2}}} .
$$

In this region both $\nu_{x}$ and $\nu_{y}$ are negative, thus at short time scales $\left(t \leq t_{c}\right)$, the linear theory (see Section VI A 1) predicts ripples oriented along the direction ( $x$ for large $a_{\sigma}$ and $y$ for small $a_{\sigma}$ ) for which the absolute value of $\nu$ is largest, with ripple wavelength $\ell=2 \pi \sqrt{\frac{2 K}{|\nu|}}$. On the other hand, at long times $\left(t \gg t_{c}\right)$, the ripple structure disappears and the surface undergoes kinetic roughening [100]. Since $\lambda_{x} \cdot \lambda_{y}>0$, we expect the dynamics of the kinetic roughening regime to be described by the
KPZ equation, i.e. the surface width follows $W \sim L^{\alpha}$, $W \sim t^{\beta}$, where the scaling exponents are $\alpha \simeq 0.38$ and $\beta \simeq 0.25$ (see Sect. IV A 1).

Region $I I-$ In this region both the $\nu_{x}$ and the $\nu_{y}$ coefficients are still negative, but in contrast with Region I the product $\lambda_{x} \cdot \lambda_{y}$ is negative. Recent studies by Park et al. 100 have shown that in time three morphological regimes can be distinguished. For short times, $t \leq t_{I}$, the ripple structure predicted by the linear theory (see Section IV A 1] is observed, with ripples oriented along the direction which has the largest value of $|\nu|$. For intermediate times $t_{I} \leq t \leq t_{I I}$, the surface is rough. If this roughness would follow kinetic roughening, one would expect logarithmic scaling, described by the EdwardsWilkinson equation (see Sections VIB 2 VIB 4), since $\lambda_{x} \cdot \lambda_{y}<0$. However, this transient regime is somewhat different from what we expect during kinetic roughening. The numerical simulations often show the development of individual ripples, which soon disappear, and no longrange order is present in the system. However, at a second crossover time, $t_{I I}$, a new ripple structure suddenly forms, in which the ripples are stable and rotated an angle $\theta_{c}$ with respect to the $x$ direction. Rost and Krug 65] have shown [for the deterministic limit of Eq. (83)] that, by defining $\alpha_{\nu}=\nu_{x} / \nu_{y}$ and $\beta_{\lambda}=\lambda_{x} / \lambda_{y}$, the fact that $\alpha_{\nu}>0$ and $\beta_{\lambda}<0$ throughout Region II implies the existence of "cancellation modes" which dominate the dynamics and lead to this rotated ripple morphology. (Note the parameter ratios $\alpha_{\nu}$ and $\beta_{\lambda}$ are not to be confused with the roughness and growth exponents $\alpha$ and $\beta$ introduced in Section III.) The angle $\theta_{c}$ calculated by Rost and Krug has the value $\theta_{c}=\tan ^{-1} \sqrt{-\lambda_{x} / \lambda_{y}}$ (see also Appendix D), and can be obtained by moving to a rotated frame of coordinates that cancels the nonlinear terms in the transverse direction. The boundary of Region $I I$ is given by the condition $\nu_{x}\left(a_{\sigma}, \theta\right)=0$, Eq. (79).

Region III - This region is characterized by a positive $\nu_{x}$ and a negative $\nu_{y}$. At short time scales, $t \leq t_{c}$, the periodic structure associated with the instability is oriented along the $y$ direction and has wavelength $\ell_{y}=2 \pi \sqrt{\frac{2 K}{\left|\nu_{y}\right|}}$. Much less is known, however, about the nonlinear regime. Such an anisotropic and linearly unstable equation is unexplored in the context of growth equations. The analysis by Rost and Krug [65] for the corresponding deterministic equation predicts that, given that $\beta_{\lambda}<\alpha_{\nu}<0$ does hold all over Region III, again cancellation modes induce a rotated ripple morphology.

Summary: Even though several aspects of the scaling behavior predicted by Eq. (83) remain to be clarified, we believe that this equation contains the relevant ingredients for understanding roughening by ion bombardment. To summarize, at short time scales the morphology consists of a periodic structure oriented along the direction determined by the largest in absolute value of the negative surface tension coefficients 38. Modifying the values of $a_{\sigma}$ or $\theta$ changes the orientation of the ripples [38]. At 
long time scales we expect two different morphological regimes. One is characterized by the KPZ exponents, which might be observed in Region $I$ in Fig. 21. Indeed, the values of the exponents reported by Eklund et al. 28,29] are consistent within the experimental errors with the KPZ exponents. In Region II kinetic roughening is not expected. Rather, nonlinear terms lead to a new ripple structure that is rotated with respect to the ion direction. Region III is less understood; analysis of the deterministic equation [65] again predicts a rotated ripple structure. By tuning the values of $\theta$ and/or $a_{\sigma}$ one may induce transitions among these morphological regimes.

\section{High temperature morphology: Asymmetric case}

In this section we discuss the effect of asymmetry on the scaling regimes of Eq. (83). Here again we obtain that the effect of asymmetry does not bring in new qualitative features. Specifically, we find that the qualitative behavior of $\xi_{x}$ and $\xi_{y}$ is not affected by the asymmetry. As the asymmetry grows, the absolute value of the coefficients in the region of small angles decreases and the peaks at large $\theta$ increase. Similarly, while the numerical values of $\lambda_{x}$ and $\lambda_{y}$ are affected by the asymmetry $\tau$, their qualitative features are not.

Finally, we find that the morphological diagram is topologically equivalent to the phase diagram obtained in the symmetric case (see Fig. 21), the only difference being in the position of the boundaries. As $\tau$ changes, we find a smooth evolution of the boundaries, which does not uncover any new regimes. Since the morphological properties of the system in the three regimes are the same as those discussed in the symmetric case, we will not discuss them again.

\section{Low temperature morphology: Symmetric case}

In this section we discuss the effect of the effective surface smoothing on the surface morphology in the nonlinear regime. In the absence of thermally activated surface diffusion, from Eq. (40) we obtain the following equation governing the morphology evolution

$$
\begin{aligned}
\frac{\partial h}{\partial t}= & -v_{0}+\gamma \frac{\partial h}{\partial x}+\xi_{x}\left(\frac{\partial h}{\partial x}\right)\left(\frac{\partial^{2} h}{\partial x^{2}}\right) \\
& +\xi_{y}\left(\frac{\partial h}{\partial x}\right)\left(\frac{\partial^{2} h}{\partial y^{2}}\right)+\nu_{x} \frac{\partial^{2} h}{\partial x^{2}} \\
& +\nu_{y} \frac{\partial^{2} h}{\partial y^{2}}+\frac{\lambda_{x}}{2}\left(\frac{\partial h}{\partial x}\right)^{2}+\frac{\lambda_{y}}{2}\left(\frac{\partial h}{\partial y}\right)^{2} \\
& +\Omega_{1} \frac{\partial^{3} h}{\partial x^{3}}+\Omega_{2} \frac{\partial^{3} h}{\partial x \partial y^{2}}-D_{x x} \frac{\partial^{4} h}{\partial x^{4}} \\
& -D_{x y} \frac{\partial^{4} h}{\partial x^{2} \partial y^{2}}-D_{y y} \frac{\partial^{4} h}{\partial y^{4}}+\eta(x, y, t) .
\end{aligned}
$$

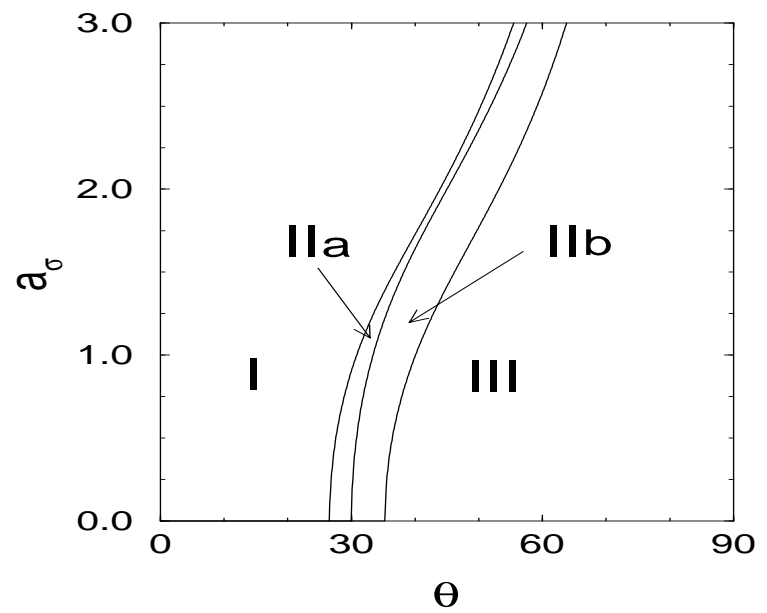

FIG. 22. Nonlinear phase diagram for the isotropic case $\sigma=\mu=1$ at low temperatures. Region I: $\nu_{x}<0, \nu_{y}<0$, $D_{x x}>0, D_{y y}>0$ and $\lambda_{x}<0, \lambda_{y}<0$; Region IIa: $\nu_{x}<0$, $\nu_{y}<0, D_{x x}<0, D_{y y}>0$ and $\lambda_{x}<0, \lambda_{y}<0$; Region IIb: $\nu_{x}<0, \nu_{y}<0, D_{x x}<0, D_{y y}>0$ and $\lambda_{x}>0, \lambda_{y}<0$; Region III: $\nu_{x}>0, \nu_{y}<0, D_{x x}<0, D_{y y}>0$ and $\lambda_{x}>0, \lambda_{y}<0$.

The terms $\gamma, \nu_{x}, \nu_{y}, \Omega_{1}, \Omega_{2}, \xi_{x}, \xi_{y}, \lambda_{x}, \lambda_{y}$, as well as the ion-induced effective smoothing coefficients $D_{x x}, D_{x y}$ and $D_{y y}$ have been discussed in the previous sections.

In the following we discuss the morphological phase diagram predicted by Eq. (89) and shown in Fig. 22. The different regimes have the following characteristics:

Region I: The surface tensions $\nu_{x}$ and $\nu_{y}$ are negative while $D_{x x}$ and $D_{y y}$ are positive, and $\lambda_{x}, \lambda_{y}$ are both negative. This regime has been previously described in Section VI B 1 (Regime I in Fig. 21), the only difference being that here the ion-induced effective surface smoothing plays the role of $K$. The boundary of this region is given by $D_{x x}\left(a_{\sigma}, \theta\right)=0$, Eq. (78).

Region IIa: Here $\nu_{x}, \nu_{y}, \lambda_{x}$, and $\lambda_{y}$ are still negative, $D_{y y}$ is positive, while $D_{x x}<0$. Consequently, along the $x$ direction the surface is unstable, all modes growing exponentially. However, the nonlinear terms $\lambda_{x}$ and $\lambda_{y}$ have the same sign. The nonlinear regime in this parameter region has not yet been explored numerically, thus its morphology is unknown. The boundary of this region is given by $\lambda_{x}\left(a_{\sigma}, \theta\right)=0$, Eq. (88).

Region IIb: In this region $D_{x x}$ is negative, $D_{y y}$ is positive, $\nu_{x}<0, \nu_{y}<0$, and $\lambda_{x}>0, \lambda_{y}<0$. Thus, the only difference of this region with respect to Region IIa is that the nonlinear terms have different signs. Similarly to Region IIa, nothing is known about the nonlinear behavior. The boundary of this region is given by $\nu_{x}\left(a_{\sigma}, \theta\right)=0$, Eq. (79).

Region III: Here we have $\nu_{x}>0, \nu_{y}<0, D_{x x}<0$, $D_{y y}>0, \lambda_{x}>0$ and $\lambda_{y}<0$. This region has similar features to Region III in the phase diagram of Fig. 21, except for the negative value of the $D_{x x}$ coefficient. 


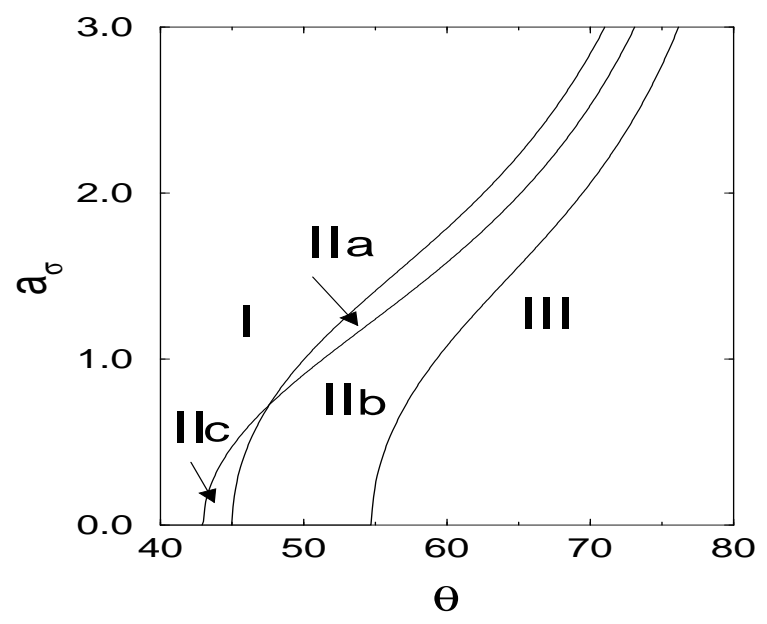

FIG. 23. Nonlinear phase diagram for the anisotropic case with $\tau=2$ and $a_{\sigma}=2$. Different regions in the diagram correspond to: Region I: $\nu_{x}<0, \nu_{y}<0, D_{x x}>0, D_{y y}>0$ and $\lambda_{x}<0, \lambda_{y}<0$; Region IIa: $\nu_{x}<0, \nu_{y}<0, D_{x x}<0$, $D_{y y}>0$ and $\lambda_{x}<0, \lambda_{y}<0$; Region IIb: $\nu_{x}<0, \nu_{y}<0$, $D_{x x}<0, D_{y y}>0$ and $\lambda_{x}>0, \lambda_{y}<0$; Region IIc: $\nu_{x}<0$, $\nu_{y}<0, D_{x x}>0, D_{y y}>0$ and $\lambda_{x}>0, \lambda_{y}<0$; Region III: $\nu_{x}>0, \nu_{y}<0, D_{x x}<0, D_{y y}>0$ and $\lambda_{x}>0, \lambda_{y}<0$.

\section{Low temperature morphology: Asymmetric case}

In this section we discuss the effect of asymmetry on the long distance properties of Eq. (89). The effect of the asymmetry on the coefficients appearing in the equation were discussed earlier. Therefore we concentrate here merely on the morphological phase diagram predicted by Eq. (89) for the asymmetric case, which is displayed in Fig. 23. As before, asymmetry $(\tau \neq 1)$ leads to a smooth shift of the boundaries of the regions provided by the lines where the coefficients $D_{x x}\left(a_{\sigma}, \theta\right), \nu_{x}\left(a_{\sigma}, \theta\right)$, and $\lambda_{x}\left(a_{\sigma}, \theta\right)$ change sign. In the presence of effective smoothing, however, asymmetry in the deposited energy distribution induces the appearance of a fifth morphological regime. This is caused by the smooth motion of the boundary determined by $\lambda_{x}\left(a_{\sigma}, \theta\right)=0$, which intersects for some value of $\tau$ the boundary defined by $D_{x x}\left(a_{\sigma}, \theta\right)=0$. Comparison of Fig. 23 and Fig. 22 illustrates how the boundaries move with $\tau$. Regions I, IIa, IIb and III are analogous of those shown in Fig. 22. Region IIc in the phase diagram, on the other hand, is analogous of Region II of the high temperature phase diagram, shown in Fig. 21, and all the conclusions obtained in that section regarding the morphological properties in this regime also apply here.

\section{COMPARISON WITH EXPERIMENTS}

In this section we compare the predictions of the theory presented in this paper with experimental results on ripple formation and surface roughening. For a better presentation, we choose to structure the material around well known features of the morphological evolution, present the theoretical predictions and discuss to which extent are they supported by the available experimental data. We also discuss predictions that have not been tested in sufficient detail but could offer future tests of the theory.

Ripple amplitude: A key quantity in ripple formation is the time evolution of the ripple amplitude. As we have shown in Section VIA1, at early times $\left(t \leq t_{c}\right)$ the ripple amplitude grows exponentially, following $h \sim$ $\exp \left(r\left(q_{x}^{*}, q_{y}^{*}\right) t\right)$, where $r$ is the growth rate of the most unstable mode $\left(q_{x}^{*}, q_{y}^{*}\right)$. According to the linear theory, this growth continues indefinitely. In contrast, the nonlinear theory predicts that the amplitude should stabilize after time $t_{c}$, where $t_{c}$ is given by Eq. (87). This is consistent with the experimental investigations 18,20 . On the other hand, recently Erlebacher et al. [19] also found that at initial stages the ripple morphology is growing exponentially. Furthermore, they observed that at some time $t_{c}$ the exponential growth stops and the ripple amplitude saturates. Measuring the temperature dependence of the saturation curves, they found that rescaling the time $t$ with a factor $\nu^{2} / 4 K$ and the amplitude $h$ with $\sqrt{\nu / 2 K}$, the different curves representing the amplitude as a function of time collapse onto a single one. This result is in excellent agreement with our prediction that suggest that plotting the result in terms of the rescaled parameters, $t / t_{c}$ and $h \sqrt{\nu / 2 K}$, the different curves should collapse [100]. They also offer direct proof that the nonlinear terms play a major role in determining the amplitude of the ripples, indicating that the incorporation of the nonlinear mechanisms in the theory of ripple formation is essential.

Temperature dependence of the ripple wavelength: A key quantity that provides direct information about the nature of the relaxation mechanism is the temperature dependence of the ripple wavelength. Our results indicate that there are two mechanisms contributing to surface relaxation: thermally induced surface diffusion (Sects. VI A 1. VI A 2) and ion-induced smoothing (Sects. VI A 3 VI A 4). At high temperatures thermal surface diffusion is rather intensive, thus it is the main mechanism determining the relaxation process, the ripple wavelength being given by 65) or (67). Since the surface diffusion constant $K$ decreases with $T$ as $(1 / T) \exp \left(-\Delta E / k_{B} T\right)$, the ripple wavelength is also expected to decrease exponentially with $T$. Indeed, such an exponential temperature dependence of $\ell$ has been observed by various groups [12,13. However, in Section VI A 3 we demonstrated the existence of ion induced smoothing, that is present at any temperature. Thus, up to some inessential numerical factors, the total surface diffusion constants have a form $D^{T}=K+D$, where $D$ is independent of temperature. Since $K$ decreases exponentially with $T$, at low enough temperatures we have $K \ll D$, indicating that 
the main relaxation mechanism is ion-induced. Consequently, below a certain critical temperature $T_{c}$, given by $K\left(T_{c}\right)=D$, one expects the ripple wavelength to be independent of $T$. Support for this scenario has been provided by the experiments in [12 and 13 and the molecular dynamics simulations of Koponen et al. [90. Consequently, as the theoretical results in this paper indicate, the temperature dependence of $\ell$ can be used to identify the relaxation mechanism: when $\ell$ increases exponentially with $T$, we are dealing with relaxation by thermal surface diffusion, while a temperature independent wavelength is an indication of ion induced smoothing.

Ripple orientation: An important feature of ripple formation is that, as the linear theory predicts, the ripple orientation depends on the angle of incidence $\theta$. The dependence of the ripple orientation on the experimental parameters has been summarized in the phase diagrams shown in Figs. 12, 15, 16, 21, 22, and 23. In general, for physical values of the asymmetry parameter (i.e. for $\tau>1$ ), we find that for small angles the ripples are oriented in the $x$ direction (along the incoming ions), and they change orientation to the $y$ direction for large $\theta$. The boundary separating these two morphological regions depends on the parameters characterizing the sputtering process, such as the ion penetration depth and the geometry of the deposited energy distribution. Such transition in the ripple orientation has been found in the simulations of [88], where for $\theta \leq 45^{\circ}$ the observed ripples were oriented along the $x$ direction, while for $\theta \geq 45^{\circ}$, they changed their orientation to $y$. Furthermore, the nonlinear theory predicts that after the nonlinear terms take over, new ripples, with orientation different from both $x$ and $y$ directions, might appear (see Sect. VIB 1). To the best of our knowledge, such rotated ripples have not been observed experimentally as yet. Nevertheless, this morphology might also lead to additional effects, such as shadowing, which have been neglected in our approach.

Ripple wavelength dependence on the flux: Depending on the nature of the relaxation mechanism, the linear theory has two different predictions on the flux dependence of the ripple wavelength: for high temperatures, when thermal surface diffusion dominates, one expects $\ell \sim J^{-1 / 2}$ [see Eq. (71)], while at low temperatures, characterized by ion induced smoothing, we expect the wavelength to be independent of flux (see Sect. VI A 3). Consequently, due to its strong dependence on the relaxation mechanism, the flux dependence of the ripple wavelength can also be used to identify the relaxation mechanism. Indeed, a number of experiments 18,13] are compatible with the prediction of a flux independent wavelength. Other aspects of ripple characteristics (such as energy or temperature dependence) also lead to the same conclusion. On the other hand, we are not aware of results indicating decreasing ripple wavelength with increasing flux. However, support for the relevance of thermal surface diffusion comes from the experiments of Chason et al. [16], who reported that the growth rate $r\left(q_{x}^{*}, q_{y}^{*}\right)$ as a function of flux follows the predictions of the linear theory with thermal surface diffusion.

Ripple wavelength dependence on the ion energy: The linear theory indicates that the ion energy dependence of the ripple wavelength can be used to distinguish between the two relaxation mechanisms: at high temperatures we expect $\ell \sim \epsilon^{-1 / 2}$ [see Eq. (70)], i.e., the wavelength decreases with the energy, while at low temperature we have $\ell \sim \epsilon^{2 m} \sim \epsilon$ [see Eq. (80)], i.e., the wavelength should increase with energy, strikingly different predictions. A number of experimental groups have found that the ripple wavelength increases linearly with the ion energy [7,9 18]. However, while we are not aware of any direct observation of a decreasing ripple wavelength with increasing ion energy, the growth rate dependence on the ion energy measured by Chason et al. [17] provided results which are in agreement with the predictions based on thermal surface diffusion.

The magnitude of the effective surface diffusion constant: Since the transition between the low and high temperature regimes is determined by the relative magnitude of $D_{x x}, D_{x y}, D_{y y}$ (ion induced smoothing), and $K$ (thermal surface diffusion), we need to estimate the magnitude of these constants. In the following we give an order of magnitude estimate for the effective surface diffusion constant $D_{x x}$ and compare it to $K$, using data from [18, 19 for $\operatorname{Si}(001)$. Taking $Y=2.6, J=670 \mu \mathrm{A} / \mathrm{cm}^{2}, \epsilon=9$ $\mathrm{keV}, a=100 \AA, a_{\sigma}=2, a_{\mu}=4$, and $\theta=40^{\circ}$, Eq. (51) gives $D_{x x} \simeq 12 \times 10^{-28} \mathrm{~cm}^{4} / \mathrm{s}$. For comparison, at $\mathrm{T}=550 \mathrm{C}$ it is estimated [19] that $K \simeq 34 \times 10^{-28}$ $\mathrm{cm}^{4} / \mathrm{s}$. Hence, since $K$ decreases exponentially with temperature, ion induced smoothing can be significant at low temperatures (including room temperature), in some cases being comparable or more relevant than thermal surface diffusion.

Kinetic roughening: An important feature of our theory is that it goes beyond the linear approach, handling systematically the nonlinear effects as well. As we demonstrated in Sect. VIB, the presence of the nonlinear terms can affect both the dynamics and the morphology of the surface. The first and the most dramatic consequence is the stabilization of the ripple amplitude, discussed above. Furthermore, after the stabilization of the ripple amplitude the surface morphology is rather different from the morphology predicted by the linear theory. In particular, depending on the signs of $\lambda_{x}$ and $\lambda_{y}$, different morphological features can develop. When $\lambda_{x} \lambda_{y}$ is positive, at large times the surface undergoes kinetic roughening, following the predictions of the KPZ equation. This behavior has, indeed, been observed experimentally 28,29] and numerically [91], providing direct support for the predictions of the nonlinear theory. When $\lambda_{x} \lambda_{y}$ is negative, direct numerical integration of the nonlinear theory 100,65 indicated the existence of a new, rotated ripple structure. The absence of experimental data on this phase might be due to the required large sputtering times: the simulations indicate 100 that between the linear regime and the formation of the rotated 
ripple structure there is a rather long transient regime with an apparently rough surface morphology. The above predictions apply when the surface diffusion terms, ion or thermally induced, act to smooth the surface (i.e., $\left.D^{T} \geq 0\right)$. However, at low temperatures, when ion induced smoothing dominates, surface diffusion can generate an instability that can further modify this behavior (see Sect. VIB 3). In general, while rather detailed experimental data are available describing the linear regime of ion sputtering, explanation of the nonlinear regime is only at its beginning, hiding the possibility of new interesting phases and behaviors.

\section{CONCLUSIONS}

In this paper we investigated the morphological properties of surfaces eroded by ion bombardment. Starting from the expression for the erosion velocity derived in the framework of Sigmund's theory of sputtering of amorphous targets, we derived a stochastic partial differential equation for the surface height, which involves up to fourth order derivatives of the height, and incorporates surface diffusion and the fluctuations arising in the erosion process due to the inhomogeneities in the ion flux. In some special cases the derived nonlinear theory reduces to the much studied KS or the KPZ equations, well known descriptions of dynamically evolving surfaces. However, in contrast with these theories, which have been derived using symmetry and conservation considerations [37, here we derived the continuum theory directly from a microscopic model of sputtering, and thus all coefficients can be explicitly expressed in terms of the physical parameters (such as angle of incidence, ion penetration depth, etc.) characterizing the ion bombardment process. An important feature of the derived nonlinear continuum theory is that the linear and the nonlinear regimes are separated in time. As a result, they can be discussed separately, the former controlling the behavior at early times, the latter at late times. Furthermore, an important result of our calculations is that higher order effects of the sputtering process can smooth the surface. This effective mechanism was necessary to explain ripple formation at low temperatures, when thermally induced surface diffusion is not relevant. Consequently, based on these two ingredients (separation of time scales between linear and the nonlinear regimes and the existence of two different relaxation mechanisms) we have discussed four different cases. In the linear high temperature regime the equations reduce to the linear theory of Bradley and Harper, predicting ripple formation, and explaining such experimentally observed phenomenon as ripple orientation (and its change with $a_{\sigma}$ and $\theta$ ), exponential increase in ripple amplitude (valid for short times), or flux and energy dependence of the ripple wavelength. On the other hand, phenomena not explained by this approach, such as the stabilization of the ripple amplitude, can be ex- plained by considering the nonlinear terms as well. We also show that, depending on the sign of the coefficients of the nonlinear terms, the late time morphology of the surface is either rough, or dominated by new rotated ripples. The rough phase is expected to be described by the KPZ equation, which has its own significance: while the introduction of the KPZ equation has catalyzed an explosion in the study of the morphological properties of growing surfaces, there are very few actual surfaces that are described by it (and not by one of its offsprings, such as the MBE or related equations [37]), and most notably none, as far as we know, that describe crystal surfaces. The sputtering problem provides one of the first systems that is convincingly described by this continuum theory. Many of the previous mysteries of low temperature ripple formation have also been solved by the present theory. By deriving the higher order ion-induced effective smoothing terms, we can explain the existence of ripples at temperatures where thermally induced surface diffusion is not active. We showed that the derived effective smoothing affects both the linear and the nonlinear regimes, governing the early time ripple formation, and the late time nonlinear behavior. The coexistence of thermal and ion induced smoothing can explain the stabilization of the ripple wavelength at low temperatures, in contrast with its exponential $T$ dependence at higher temperatures. On the other hand, our theory has limitations, most of which can be already identified in Sigmund's and Bradley and Harper's theories, with which it is related. Namely, it is devised for amorphous substrates, whereupon it neglects effects such as viscous relaxation [7], which might be the cause for the failure of the theory to predict the absence in many experiments of ripples at low (but non-zero) angles of incidence. This issue should constitute one of the most important extensions of our present theory. Perhaps related with this, we have seen that there exist parameter regions at low temperatures within which our theory breaks down, due to the unstable higher order derivative terms that occur. A relevant issue is thus to determine the correct continuum description of the surface under these conditions.

Most of the predictions offered by the presented continuum theory have been already verified experimentally. However, many unexplained predictions remain at low temperatures both in the linear and the nonlinear regimes, as well as regarding the nonlinear regime at high temperatures. We hope that the rather precise derivations offered in this paper will guide such future experimental work. Furthermore, some of the morphologies expected in the nonlinear regime need further theoretical understanding as well, allowing for the continuation of this inquiry. With the dramatic advances in computer speed, the understanding of some of these questions, either through numerical integration of the continuum theory or through discrete models, might be not too far. 


\section{ACKNOWLEDGMENTS}

We would like to acknowledge discussions with E. Chason, B. Kahng, H. Jeong, F. Ojeda, and L. Vázquez. This research was supported by NSF-DMR CAREER and ONR-YI awards (A.-L. B. and M. M.) and DGES (Spain) grant PB96-0119 (R. C.).

\section{APPENDIX A:}

The algebraic relation between the coordinates of the laboratory frame and the local frame, depicted in Fig. 3. follows from the definitions given in point (i) of Sect. V. Accordingly, the unit vector along the $\hat{Z}$ axis is the normal at point $O$

$$
\hat{Z} \equiv \hat{n}=\frac{\left(-\partial_{x} h,-\partial_{y} h, 1\right)}{\sqrt{g}},
$$

where $g \equiv 1+\left(\partial_{x} h\right)^{2}+\left(\partial_{y} h\right)^{2}$. The vector $\hat{m}$ drawn on Fig. 3 has components

$$
\hat{m}=(\sin \theta, 0, \cos \theta) .
$$

Therefore, the unit vector along the $\hat{Y}$ axis reads:

$$
\begin{aligned}
\hat{Y} \equiv & \frac{\hat{n} \times \hat{m}}{|\hat{n} \times \hat{m}|}=\frac{1}{\sqrt{g} \sin \varphi}\left(-\left(\partial_{y} h\right) \cos \theta, \sin \theta\right. \\
& \left.+\left(\partial_{x} h\right) \cos \theta,\left(\partial_{y} h\right) \sin \theta\right),
\end{aligned}
$$

and finally (A1) and (A3) yield for the unit vector along the $\hat{X}$ axis:

$$
\begin{aligned}
\hat{X}= & \hat{Y} \times \hat{Z} \\
= & \frac{1}{g \sin \varphi}\left(\sin \theta+\left(\partial_{x} h\right) \cos \theta\left(\partial_{y} h\right)^{2} \sin \theta,\right. \\
& \left(\partial_{y} h\right) \cos \theta-\left(\partial_{x} h\right)\left(\partial_{y} h\right) \sin \theta, \\
& \left.\left(\partial_{x} h\right) \sin \theta+\left(\left(\partial_{x} h\right)^{2}+\left(\partial_{y} h\right)^{2}\right) \cos \theta\right) .
\end{aligned}
$$

The matrix $\mathcal{M}$ defined in Eq. (36) and which relates the coordinates in the local and laboratory frames reads

$$
\mathcal{M}=\left(\begin{array}{ccc}
\frac{s+c \partial_{y} h+s\left(\partial_{y} h\right)^{2}}{\sqrt{g} r} & -\frac{c \partial_{y} h}{r} & -\frac{\partial_{x} h}{\sqrt{g}} \\
\frac{c \partial_{y} h-s \partial_{x} h \partial_{y} h}{\sqrt{g} r} & \frac{s+c \partial_{x} h}{r} & -\frac{\partial_{y} h}{\sqrt{g}} \\
\frac{s \partial_{x} h-c\left(\left(\partial_{x} h\right)^{2}+\left(\partial_{y} h\right)^{2}\right)}{\sqrt{g} r} & \frac{s \partial_{y} h}{r} & -\frac{1}{\sqrt{g}}
\end{array}\right),
$$

where $s=\sin \theta, c=\cos \theta$, and

$r \equiv \sqrt{\left(s+c \partial_{x} h\right)^{2}+\left(\partial_{y} h\right)^{2}}$.

\section{APPENDIX B:}

If we perform a small $\Delta_{n m}$ expansion in Eq. (30) we obtain

$$
\begin{aligned}
V_{O} & =\frac{\epsilon p J a^{2}}{\sigma \mu(2 \pi)^{3 / 2}} \exp \left(-a_{\sigma}^{2} / 2\right) \\
& \times \int_{-\infty}^{\infty} \int_{-\infty}^{\infty} d \zeta_{X} d \zeta_{Y} \exp \left(-\zeta_{Y} L^{2}\right) \\
& \times \exp \left(-\zeta_{X} A-\frac{1}{2} \zeta_{X}^{2} B_{1}\right)\left[\operatorname { c o s } \varphi \left(1+B_{2} \Delta_{20} \zeta_{X}^{2}\right.\right. \\
& +B_{2} \Delta_{02} \zeta_{Y}^{2}+B_{2} \Delta_{30} \zeta_{X}^{3}+B_{2} \Delta_{12} \zeta_{X} \zeta_{Y}^{2} \\
& +B_{2} \Delta_{22} \zeta_{X}^{2} \zeta_{Y}^{2}+B_{2} \Delta_{40} \zeta_{X}^{4}+B_{2} \Delta_{04} \zeta_{Y}^{4} \\
& -2 C \Delta_{20} \zeta_{X}^{3}-2 C \Delta_{02} \zeta_{Y}^{2} \zeta_{X}-2 C \Delta_{30} \zeta_{X}^{4} \\
& -2 C \Delta_{12} \zeta_{X}^{2} \zeta_{Y}^{2}-2 C \Delta_{22} \zeta_{X}^{3} \zeta_{Y}^{2}-2 C \Delta_{40} \zeta_{X}^{5} \\
& \left.-2 C \Delta_{04} \zeta_{X} \zeta_{Y}^{4}\right)+\sin \varphi\left(2 \Delta_{20} \zeta_{X}+3 \Delta_{30} \zeta_{X}^{2}\right. \\
& \left.\left.+\Delta_{12} \zeta_{Y}^{2}+2 \Delta_{22} \zeta_{X} \zeta_{Y}^{2}+4 \Delta_{40} \zeta_{X}^{3}\right)\right] .
\end{aligned}
$$

Evaluating the Gaussian integrals in this formula we obtain Eq. (32), where the coefficients $\Gamma_{n m}(\varphi)$ are given by

$$
\begin{aligned}
& \Gamma_{20}(\varphi)=-\frac{2 A}{B_{1}} \sin \varphi+\frac{B_{2}}{B_{1}}\left[1+\frac{A^{2}}{B_{1}}\right] \cos \varphi \\
& +\frac{2 A C}{B_{1}^{2}}\left[3+\frac{A^{2}}{B_{1}}\right] \cos \varphi \\
& \Gamma_{02}(\varphi)=2 \frac{\mu^{2}}{a^{2}} \cos \varphi\left(\frac{B_{2}}{2}+\frac{A C}{B_{1}}\right) \text {, } \\
& \Gamma_{30}(\varphi)=\sin \varphi\left(\frac{1}{B_{1}}+\frac{A^{2}}{B_{1}^{2}}\right)-B_{2} \cos \varphi\left(\frac{3 A}{B_{1}^{2}}+\frac{A^{3}}{B_{1}^{3}}\right) \\
& -2 C \cos \varphi\left(\frac{3}{B_{1}^{2}}+\frac{6 A^{2}}{B_{1}^{3}}+\frac{A^{4}}{B_{1}^{4}}\right) \text {, } \\
& \Gamma_{12}(\varphi)=2 \frac{\mu^{2}}{a^{2}}\left\{\sin \varphi-B_{2} \cos \varphi \frac{A}{B_{1}}\right. \\
& \left.-2 C \cos \varphi\left(\frac{1}{B_{1}}+\frac{A^{2}}{B_{1}^{2}}\right)\right\} \text {, } \\
& \Gamma_{40}(\varphi)=\left\{4 \sin \varphi\left(\frac{-3 A}{B_{1}^{2}}-\frac{A^{3}}{B_{1}^{3}}\right)\right. \\
& +B_{2} \cos \varphi\left(\frac{3}{B_{1}^{2}}+\frac{6 A^{2}}{B_{1}^{3}}+\frac{A^{4}}{B_{1}^{4}}\right) \\
& \left.+2 C \cos \varphi\left(\frac{15 A}{B_{1}^{3}}+\frac{10 A^{3}}{B_{1}^{4}}+\frac{A^{5}}{B_{1}^{5}}\right)\right\} \text {. } \\
& \Gamma_{22}(\varphi)=2 \frac{\mu^{2}}{a^{2}}\left\{-2 \sin \varphi \frac{A}{B_{1}}+B_{2} \cos \varphi\left(\frac{1}{B_{1}}+\frac{A^{2}}{B_{1}^{2}}\right)\right. \\
& \left.+2 C \cos \varphi\left(\frac{3 A}{B_{1}^{2}}+\frac{A^{3}}{B_{1}^{3}}\right)\right\} \text {. } \\
& \Gamma_{04}(\varphi)=3 \frac{\mu^{4}}{a^{4}}\left\{B_{2} \cos \varphi+2 C \cos \varphi \frac{A}{B_{1}}\right\} \text {. }
\end{aligned}
$$

Taking into account Eqs. (33a), (33b) relating the local $(\varphi)$ and the global $(\theta)$ angles of incidence through the surface slopes $\partial_{x} h, \partial_{y} h$, a small slope approximation leads to 


$$
\begin{aligned}
e^{A^{2} / 2 B_{1}} & \simeq e^{a_{\sigma}^{4} s^{2} / 2 f}\left\{1+\frac{a_{\sigma}^{4}}{2 f}\left[\frac{a_{\mu}^{2}}{f} s\left(\partial_{x} h\right)+\frac{a_{\mu}^{2}}{f} c^{2}\left(\partial_{y} h\right)^{2}\right.\right. \\
& +\frac{a_{\mu}^{2}}{f^{2}}\left(\partial_{x} h\right)^{2}\left(a_{\mu}^{2} c^{2}\left(1+2 s^{2}\right)-a_{\sigma}^{2} s^{2}\left(1+2 c^{2}\right)\right. \\
& \left.\left.\left.+\frac{a_{\sigma}^{4} a_{\mu}^{2}}{f} s^{2} c^{2}\right)\right]\right\} \\
B_{1}^{-1 / 2} & \simeq \frac{1}{\sqrt{f}}\left\{1-\frac{a_{\sigma}^{2}-a_{\mu}^{2}}{f} s c\left(\partial_{x} h\right)\right. \\
& +\frac{a_{\sigma}^{2}-a_{\mu}^{2}}{2 f^{2}}\left(\partial_{x} h\right)^{2}\left(a_{\sigma}^{2} s^{2}\left(1+c^{2}\right)\right. \\
& \left.\left.-a_{\mu}^{2} c^{2}\left(1+s^{2}\right)\right)-\frac{a_{\sigma}^{2}-a_{\mu}^{2}}{2 f} c^{2}\left(\partial_{y} h\right)^{2}\right\} .
\end{aligned}
$$

Also, we have

$$
\begin{aligned}
\Gamma_{20}(\theta) & =\Gamma_{20}^{(0)}(\theta)+\Gamma_{20}^{(1)}(\theta)\left(\partial_{x} h\right), \\
\Gamma_{02}(\theta) & =\Gamma_{02}^{(0)}(\theta)+\Gamma_{02}^{(1)}(\theta)\left(\partial_{x} h\right), \\
\Gamma_{20}^{(0)}(\theta) & =\frac{a_{\sigma}^{2}}{2 f^{3}}\left\{-2 a_{\sigma}^{6} s^{4} c^{2}+2 a_{\sigma}^{4} s^{4}\left(s^{2}-2 c^{2}\right)\right. \\
& +a_{\sigma}^{4} a_{\mu}^{2} s^{2} c^{2}\left(s^{2}-c^{2}\right)+a_{\sigma}^{2} a_{\mu}^{2} \\
& \left.\times s^{2} c^{2}\left(7 s^{2}-5 c^{2}\right)+a_{\mu}^{4} c^{4}\left(5 s^{2}-c^{2}\right)\right\}, \\
\Gamma_{20}^{(1)}(\theta) & =\frac{a_{\sigma}^{2} s c}{f^{4}}\left\{-2 a_{\sigma}^{6} s^{4}-a_{\mu}^{6} c^{4}+a_{\sigma}^{6} a_{\mu}^{2}\right. \\
& \times\left(s^{2}+s^{2} c^{2}\right)-a_{\sigma}^{4} a_{\mu}^{4}\left(c^{2}+c^{2} s^{2}\right) \\
& \left.+a_{\sigma}^{4} a_{\mu}^{2}\left(5 s^{2}-3 s^{2} c^{2}\right)+4 a_{\sigma}^{2} a_{\mu}^{4} c^{2}\right\}, \\
\Gamma_{02}^{(0)}(\theta) & =-\frac{c^{2} a_{\sigma}^{2}}{2 f} \\
\Gamma_{02}^{(1)}(\theta) & =\frac{a_{\sigma}^{4} c s}{f^{2}} .
\end{aligned}
$$

In the above expressions we used the notations

$$
\begin{aligned}
& a_{\sigma} \equiv \frac{a}{\sigma}, a_{\mu} \equiv \frac{a}{\mu}, s \equiv \sin \theta, \\
& c \equiv \cos \theta, f \equiv a_{\sigma}^{2} s^{2}+a_{\mu}^{2} c^{2} .
\end{aligned}
$$

\section{APPENDIX C:}

Equations (34a)-(34b) relating the incidence angle as measured in the local and laboratory reference frames apply only in the off-normal incidence case $(\theta \neq 0)$. In the following we derive the correct expressions for the normal incidence case $(\theta=0)$. Indeed, if $\theta=0$, the vectors $\hat{n}$ and $\hat{m}$ shown in Fig. 3 are given by

$$
\hat{n}=\frac{\left(-\partial_{x} h,-\partial_{y} h, 1\right)}{\sqrt{g}}, \hat{m}=(0,0,1) .
$$

Proceeding now as in (33a)-(33b), we obtain

$$
\cos \varphi=\frac{1}{\sqrt{g}}, \quad \sin \varphi=\sqrt{\frac{\left(\partial_{x} h\right)^{2}+\left(\partial_{y} h\right)^{2}}{g}},
$$

which are the $\theta \rightarrow 0$ limit of Eqs. (33a and $(33 \mathrm{~b})$. The small gradient expansion performed on Eq. (C2) now gives

$$
\begin{aligned}
& \cos \varphi \simeq 1-\frac{1}{2}\left(\left(\partial_{x} h\right)^{2}+\left(\partial_{y} h\right)^{2}\right), \\
& \sin \varphi \simeq \sqrt{\left(\partial_{x} h\right)^{2}+\left(\partial_{y} h\right)^{2}} .
\end{aligned}
$$

Using Eqs. (C3) in the expansions leading to Eq. (40), it can be seen that the expressions obtained for the coefficients indeed are the $\theta \rightarrow 0$ limit of Eqs. (41)-(55).

\section{APPENDIX D:}

The solution corresponding to a rotated ripple structure follows from Eq. (83). Indeed, in the absence of the $\xi_{x}$ and $\xi_{y}$ terms, if we consider a solution of (83) of the form $h(x, y, t)=g(x-v y, t)$ with $v$ an arbitrary constant, the surface morphology evolution equation takes the form

$$
\begin{aligned}
\partial_{t} g= & -v_{0}+\gamma \partial_{l} g+\left(\nu_{x}+v^{2} \nu_{y}\right) \partial_{l}^{2} g \\
& +\frac{1}{2}\left(\lambda_{x}+v^{2} \lambda_{y}\right)\left(\partial_{l} g\right)^{2}+\left(\Omega_{1}+v^{2} \Omega_{2}\right) \partial_{l}^{3} g \\
& -K\left(1+v^{2}\right)^{2} \partial_{l}^{4} g
\end{aligned}
$$

where $g(l)=g(x-v y)$ is the steady wave solution [104]. From (D1) it follows that the nonlinearity vanishes when $\lambda_{x}+v^{2} \lambda_{y}=0$, or $v=\sqrt{-\lambda_{x} / \lambda_{y}}$. In this case we obtain an exponentially growing ripple structure with ripples forming an angle $\theta_{c}=\tan ^{-1}(v)=\tan ^{-1}\left(\sqrt{-\lambda_{x} / \lambda_{y}}\right)$ with respect to the $x$ axis.

[1] P. D. Townsend, J. C. Kelly, and N. E. W. Hartley, Ion Implantation, Sputtering and their Applications (Academic Press Inc, London, 1976).

[2] Sputtering by Particle Bombardment, R. Behrisch, ed. (Springer-Verlag, Heidelberg, 1981, 1983), Vols. I, II.

[3] W. Eckstein, Computer Simulation of Ion-Solid Interactions (Springer-Verlag, Heidelberg, 1991).

[4] D. J. Barber, F. C. Frank, M. Moss, J. W. Steeds, and I. S. Tsong, J. Mater. Sci. 8, 1030 (1973).

[5] F. Vasiliu, I. A. Teodorescu, and F. Glodeanu, J. Mater. Sci. 10, 399 (1975).

[6] G. Carter, B. Navinšek, and J. L. Whitton in Vol. II of Ref. [2], p. 231. 
[7] F. A. Stevie, P. M. Kahora, D. S. Simons, and P. Chi, J. Vac. Sci. Tech. A 6, 76 (1988).

[8] A. Ishitani, A. Karen, Y. Nakagawa, M. Uchida, M. Hatada, K. Okuno, and F. Soeda, in Proceedings of the 8th International Conference on Secondary Ion Mass Spectroscopy, SIMS VIII, edited by A. Benninghoven, K. T. F. Jansen, J. Tümpner, and H. W. Werner (Wiley, Chichester, 1992).

[9] A. Karen, K. Okuno, F. Soeda, and A. Ishitani, J. Vac. Sci. Technol. A 4, 2247 (1991).

[10] A. Karen, Y. Nakagawa, M. Hatada, K. Okuno, F. Soeda, and A. Ishitani, Surf. Interface Anal., 23, 506 (1995).

[11] K. Wittmaack, J. Vac. Sci. Thechnol. A 8, 2246 (1990).

[12] S. W. MacLaren, J. E. Baker, N.L. Finnegan, and C. M. Loxton, J. Vac. Sci. Technol. A 10, 468 (1992).

[13] C. C. Umbach, R. L. Headrick, B. H. Cooper, J.M. Balkely, and E. Chason, Bull. Am. Phys. Soc., 44, No 1, 706 (1999).

[14] G. Carter and V. Vishnyakov, Phys. Rev. B 54, 17647 (1996).

[15] E. Chason, T. M. Mayer, B. K. Kellerman, D. T. McIlroy, and A. J. Howard, Phys. Rev. Lett. 72, 3040 (1994).

[16] T. M. Mayer, E. Chason, and A. J. Howard, J. Appl. Phys. 76, 1633 (1994).

[17] E. Chason, T. M. Mayer, and B. K. Kellerman, Mat. Res. Soc. Symp. Proc. 396, 143 (1996).

[18] J. J. Vajo, R. E. Doty, and E.-H. Cirlin, J. Vac. Sci. Technol. A 14, 2709 (1996).

[19] J. Erlebacher, M. Aziz, E. Chason, M. Sinclair, and J. Floro, Phys. Rev. Lett. 82, 2330 (1999).

[20] J. Erlebacher, M. Aziz, E. Chason, M. B. Sinclair, and J. Floro, (to be published).

[21] K. Elst, W. Vandervorst, J. Alay, J. Snauwaert, and L. Hellemans, J. Vac. Sci. Technol. A 11, 1968 (1993).

[22] K. Elst and W. Vandervorst, J. Vac. Sci. Technol. A 12, 3205 (1994).

[23] H. Shichi, K. Ohnishi, and S. Nomura, Jpn. J. Appl. Phys. 30, L927 (1991).

[24] S. Rusponi, C. Boragno, and U. Valbusa, Phys. Rev. Lett. 78, 2795 (1996).

[25] S. Rusponi, G. Costantini, C. Boragno, and U. Valbusa, Phys. Rev. Lett. 81, 4184 (1998).

[26] E.-H. Cirlin, J. J. Vajo, R. E. Doty, and T. C. Hasenberg, J. Vac. Sci. Technol. A 9, 1395 (1991).

[27] E.-H. Cirlin, Thin Solid Films, 220, 197 (1992).

[28] E. A. Eklund, R. Bruinsma, J. Rudnick, and R. S. Williams, Phys. Rev. Lett. 67, 1759 (1991).

[29] E. A. Eklund, E. J. Snyder, and R. S. Williams, Surf. Sci. 285, 157 (1993).

[30] J. Krim, I. Heyvaert, C. Van Haesendonck, and Y. Bruynseraede, Phys. Rev. Lett. 70, 57 (1993).

[31] H.-N. Yang, G.-C. Wang, and T.-M. Lu, Phys. Rev. B 50, 7635 (1994).

[32] S.-X. Wang, R. J. Pechman, and J. H. Wearer, Surf. Sci. 364, L511 (1996).

[33] D.-M. Smilgies, P. J. P. J. Eng, E. Landemark, and M. Nielsen, Surf. Sci. 377, 1038 (1997).

[34] A. C.-T. Chan and G.-C. Wang, Surf. Sci. 414, 17 (1998).
[35] P. Konarski and M. Hautala, Vacuum 47, 1111 (1996).

[36] Z. Csahók, Z. Farkas, M. Menyhárd, G. Gergely, Cs. S. Daróczi, Surf. Sci. 364, L600 (1996).

[37] A.-L. Barabási and H. E. Stanley, Fractal Concepts in Surface Growth (Cambridge University Press, Cambridge, 1995); Dynamics of Fractal Surfaces, F. Family and T. Vicsek, eds. (World Scientific, Singapore, 1991); P. Meakin, Phys. Rep. 235, 189 (1993); T. HalpinHealey and Y.-C. Zhang, Phys. Rep. 254, 215 (1995).

[38] R. M. Bradley and J. M. E. Harper, J. Vac. Sci. Technol. A 6, 2390 (1988).

[39] R. Cuerno and A.-L. Barabási, Phys. Rev. Lett. 74, 4746 (1995).

[40] M. Makeev and A.-L. Barabási, Appl. Phys. Lett. 71, 2800 (1997).

[41] F. Family and T. Vicsek, J. Phys. A 18, L15 (1985).

[42] M. Kardar, G. Parisi, and Y.-C. Zhang, Phys. Rev. Lett. 56, 889 (1986).

[43] S. J. Chey, J. E. Nostrand, and D. G. Cahill, Phys. Rev. B, 52, 166961995.

[44] P. Šmilauer, M. R. Wilby, and D. D. Vvedensky, Phys. Rev. B 48, R4968 (1993).

[45] P. Šmilauer, M. R. Wilby, and D. D. Vvedensky, Surf. Sci. Lett. 291, L733 (1993).

[46] E. Chason, P. Bedrossian, J. E. Houston, J. Y. Tsao, B. W. Dodson, and S. T. Picraux, Appl. Phys. Lett. 59, 3533 (1991).

[47] H. Watanabe and M. Ichikawa, Appl. Phys. Lett. 68, 2514 (1996).

[48] B. Poelsema, L. Verheij, and G. Comsa, Jpn. J. Appl. Phys. 33, 2244 (1994).

[49] P. Bedrossian, J. E. Houston, J. Y. Tsao, E. Chason, and S. T. Picraux, Phys. Rev. Lett. 67, 124 (1991).

[50] J. M. Kim and J. M. Kosterlitz, Phys. Rev. Lett. 62, 2289 (1989); B. M. Forrest and L. Tang, J. Stat. Phys. 60, 181 (1990); J.G. Amar and F. Family, Phys. Rev. A 41, 3399 (1990); K. Moser, D. E. Wolf, and J. Kertész, Physica A 178, 215 (1991); T. Ala Nissila, T. Hjelt, J. M. Kosterlitz, and O. Venäläinen, J. Stat. Phys. 72, 207 (1993).

[51] S. F. Edwards and D. R. Wilkinson, Proc. R. Soc. London A 381, 17 (1982).

[52] J. Villain, J. Phys. I (France) 1, 19 (1991).

[53] D. E. Wolf, Phys. Rev. Lett. 67, 1783 (1991).

[54] The relevance of the AKPZ equation for ion-sputtered surfaces was first pointed out by R. Bruinsma in Surface Disordering: Growth, Roughening and Phase Transitions, edited by R. Jullien, J. Kertész, P. Meakin, and D. E. Wolf (Nova Science, New York, 1992). Note, however, that the AKPZ equation does not have any instability and thus is unable to predict the observed ripple structure.

[55] Y. Kuramoto and T. Tsuzuki, Prog. Theor. Phys. 55, 356 (1977); G. I. Sivashinsky, Acta Astronaut. 6, 569 (1979).

[56] V. S. L'vov, V. V. Lebedev, M. Paton, and I. Procaccia, Nonlinearity 6, 25 (1993).

[57] V. Yakhot, Phys. Rev. A 24, 642 (1981).

[58] S. Zaleski, Physica D 34, 427 (1989).

[59] F. Hayot, C. Jayaprakash, and Ch. Josserand, Phys. 
Rev. E 47, 911 (1993).

[60] K. Sneppen, J. Krug, M. H. Jensen, C. Jayaprakash, and T. Bohr, Phys. Rev. A 46, R7351 (1992).

[61] V. L'vov and I. Procaccia, Phys. Rev. Lett. 69, 3543 (1992); ibid. 72, 302 (1994).

[62] C. C. Chow and T. Hwa, Physica D 84, 494 (1995).

[63] I. Procaccia, M. Jensen, V. L'vov, K. Sneppen, and R. Zeitak, Phys. Rev. A 46, 3220 (1992).

[64] C. Jayaprakash, F. Hayot, and R. Pandit, Phys. Rev. Lett. 71, 12 (1993); ibid. 72, 308 (1994).

[65] M. Rost and J. Krug, Phys. Rev. Lett. 75, 3894 (1995).

[66] L. Golubović and R. Bruinsma, Phys. Rev. Lett. 66, 321 (1991); ibid. 67, 2747 (E) (1991).

[67] A. Karma and C. Misbah, Phys. Rev. Lett. 71, 3810 (1993).

[68] R. Cuerno and K. B. Lauritsen, Phys. Rev. E 52, 4853 (1995).

[69] P. Sigmund, Phys. Rev. 184, 383 (1969).

[70] P. Sigmund, J. Mat. Sci. 8, 1545 (1973).

[71] S. Rusponi, G. Costantini, C. Boragno, U. Valbusa, Phys. Rev. Lett. 81, 2735 (1998).

[72] C. Herring, J. Appl. Phys. 21, 301 (1950).

[73] W. W. Mullins, J. Appl. Phys. 28, 333 (1957).

[74] Another prominent smoothing mechanism is viscous relaxation, which in amorphous materials is fully determined by the mobility of defects. This mechanism has been studied in the context of ripple formation by Chason et al. [16], showing that it reduces the amplitude of the high frequency perturbations to the extent that a large viscous relaxation rate may even suppress the ripple structure altogether. In the absence of a clear understanding of the structure and dynamics of radiation induced defects, at this point it is not possible to fully quantify the effect of viscous relaxation 16. Related relaxation mechanisms have been proposed, based on defect motion and recombination, A. Witvrouw and F. Spaepen, J. Appl. Phys. 74, 7154 (1993), or defect creation, C. A. Volkert, J. Appl. Phys. 70, 3521 (1991).

[75] M. Marsili, A. Maritan, F. Toigo, and J. R. Banavar, Rev. Mod. Phys. 68, 963 (1996).

[76] D. E. Wolf and J. Villain, Europhys. Lett. 13, 389 (1990).

[77] S. Das Sarma and P. I. Tamborenea, Phys. Rev. Lett. 66, 325 (1991).

[78] V.O. Babaev, Ju.V. Bukov, and M.B. Guseva, Thin Solid Films 38, 15 (1976).

[79] M. Marinov, Thin Solid Films 46, 267 (1977).

[80] S.A. Barnett, H.F. Winters, and J.E. Greene, Surf. Sci. 181; Zh.I. Dranova and I.M. Mikhailovskii, Sov. Phys.Solid State 12, 104 (1970); E. Chason, P. Bedrossian, K. M. Horn, J. Y. Tsao, and S. T. Picraux, Appl. Phys. Lett. 57, 1793 (1990); E. Kay and S. M. Rossnagel, in [2].
[81] J.Y. Cavaille and M. Drechsler, Surf. Sci. 75, 342 (1978).

[82] S.M. Rossnagel, R.S. Robinson, and H.R. Kaufman, Surf. Sci. 123, 89 (1982).

[83] R.S. Robinson, S.M. Rossnagel, J. Vac. Sci. Technol. 21, 790 (1982).

[84] A.N. Protsenko, Nucl. Inst. Meth. in Phys. Res. B 82, 417 (1993).

[85] M. Hautala and I. Koponen, Nucl. Instr. and Meth. in Phys. Res. B 117, 95 (1996).

[86] I. Koponen, M. Hautala, and O.-P. Sievänen, Phys. Rev. B 54, 13502 (1996).

[87] I. Koponen, M. Hautala, and O.-P. Sievänen, Nucl. Instr. and Meth. in Phys. Res. B 127/128, 230 (1997).

[88] I. Koponen, M. Hautala, and O.-P. Sievänen, Phys. Rev. Lett. 78, 2612 (1997).

[89] I. Koponen, O.-P. Sievänen, M. Hautala, and M. Hakovirta, J. Appl. Phys. 82, 6047 (1997).

[90] I. Koponen, M. Hautala, and O.-P. Sievänen, Nucl. Instr. and Meth. in Phys. Res. B 129, 349 (1997).

[91] R. Cuerno, H. Makse, S. Tomassone, S. Harrington, and H. E. Stanley, Phys. Rev. Lett. 75, 4464 (1995).

[92] S. Tomassone, H. A. Makse, and R. Cuerno (unpublished).

[93] A.-L. Barabási, M. Makeev, C.-S. Lee, and R. Cuerno, in Dynamics of Fluctuating Interfaces and Related Phenomena, 4th CTP Workshop on Statistical Physics, Seoul, Korea, edited by D. Kim, H. Park, and B. Kahng (World Scientific, Singapore, 1997).

[94] K. B. Lauritsen, R. Cuerno, and H. A. Makse, Phys. Rev. E 54, 3577 (1996).

[95] D. D. Vvedesnky, A. Zangwill, C. N. Luse, and M. R. Wilby, Phys. Rev. E 48, 852 (1993).

[96] S. Wintenrbom, Rad. Effects 45, 187 (1967).

[97] M. Makeev and A.-L. Barabási, Appl. Phys. Lett. 72, 906 (1998).

[98] M. Makeev and A.-L. Barabási, Appl. Phys. Lett. 73, 1445 (1998).

[99] It has been recently shown in G. Carter, Phys. Rev. B 59, 1669 (1999) that incorporation of terms of higher order than those appearing in Eq. (21) does not substantially change the qualitative conclusions to be discussed in VI A 1 1 VI B 2.

[100] S. Park, B. Kahng, H. Jeong, and A.-L. Barabasi, (to be published).

[101] G. Carter, M. J. Nobes, I. V. Katardjiev, Philos. Mag. B 68, 231 (1993).

[102] For a review see G. S. Bales, R. Bruinsma, E. A. Eklund, R. P. U. Karunasiri, J. Rudnick, and A. Zangwill, Science 249, 264 (1990).

[103] R. Cuerno, (unpublished).

[104] A. N. Samarsky and V. T. Tikhonov, Equations of Mathematical Physics (Macmillan, New York, 1963). 